$$
U C R L-I D--116335
$$

\title{
Scientific Applications for High-Energy Lasers
}

March 1, 1994

Compiled by: Richard W. Lee University of California Lawrence Livermore National Laboratory P.O. Box 808 L-58

Livermore CA 94551

Phone: 510 422-7209

Fax: 510 422-5102 email: dicklee@llnl.gov 


\section{DISCLAIMER}

This document was prepared as an account of work sponsored by an agency of the United States Government. Neither the United States Government nor the University of California nor any of their employees, makes any warranty, express or implied, or assumes any legal liability or responsibility for the accuracy, completeness, or usefulness of any information, apparatus, product, or process disclosed, or represents that its use would not infringe privately owned rights. Reference herein to any specific commercial products, process, or service by trade name, trademark, manufacturer, or otherwise, does not necessarily constitute or imply its endorsement, recommendation, or favoring by the United States Government or the University of California. The views and opinions of authors expressed herein do not necessarily state or reflect those of the United States Government or the University of California, and shall not be used for advertising or product endorsement purposes.

This report has been reproduced directly from the best available copy.

Available to DOE and DOE contractors from the Office of Scientific and Technical Information P.O. Box 62, Oak Ridge, TN 37831

Prices available from (615) 576-8401, FTS 626-8401

Available to the public from the National Technical Information Service

U.S. Department of Commerce 5285 Port Royal Rd., Springfield, VA 22161

This is an informal report intended primarily for internal or limited external distribution. The opinions and conclusions stated are those of the author and may or may not be those of the Laboratory.

Work performed under the auspices of the U.S. Department of Energy by Lawrence Livermore National Laboratory under Contract W-7405-Eng-48. 


\title{
Scientific Applications for \\ High-Energy Lasers
}

\author{
March 1, 1994
}

\begin{abstract}
The convergence of numerous factors makes the time ripe for the development of a community of researchers to use the high-energy laser for scientific investigations. This document attempts to outline the steps necessary to access high-nergy laser systems and create a realistic plan to implement usage. Since an academic/scientific user community does not exist in the USA to any viable extent, we include information on on present capabilities at the Nova laser. This will briefly cover laser performance and diagnostics and a sampling of some current experimental projects. Further, to make the future possibilities clearer, we will describe the proposed next-generation high-energy laser, named for its inertial fusion confinement (ICF) goal, the multi-megaJoule, 500-teraWatt National Ignition Facility, or NIF.
\end{abstract}




\section{Contents}

I) Preamble ............................................................................................................1

II) Definition of the Existing Facility .......................................................

A) Introduction ............................................................................................................................ 2

B) Laser Beams ................................................................................................................... 2

C) Beam Pointing, Aiming and Synchronicity ............................................................... 3

D) Laser Pulse Shapes ......................................................................................................... 3

E) Laser Shot Rate ............................................................................................................... 3

F) Beam Diagnostics ..................................................................................................................... 3

III) Experimental Capabilities and Techniques ......................................4

A) Introduction and Overview ......................................................................................... 4

B) Atomic Physics ........................................................................................................................ 7

C) Plasma Spectroscopy ................................................................................................ 9

D) Radiative Transfer .......................................................................................................... 12

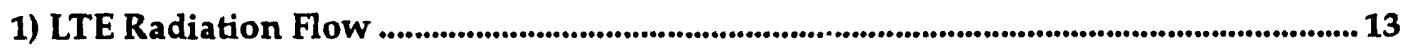

2) Non-LTE Radiative Transfer ........................................................................................................... 15

E) X-ray Lasers ................................................................................................................. 18

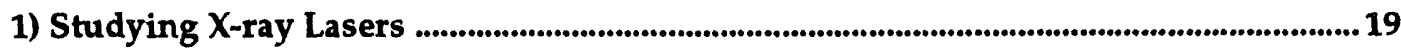

2) Development of X-ray Lasers As Probes ...................................................................20

F) Material Properties ...................................................................................................... 25

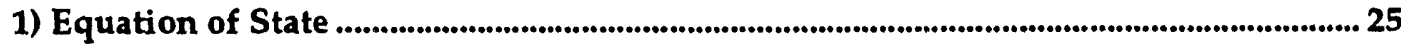

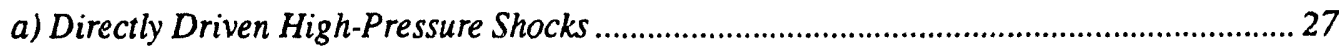

b) Indirectly Driven Colliding Foil Experiments ....................................................... 28

c) Indirectly Driven Shock Experiments on Polystyrene .................................................. 31

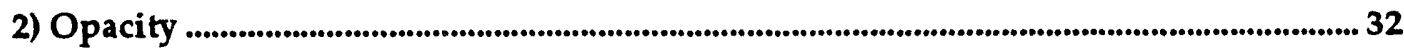

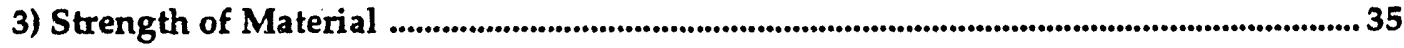

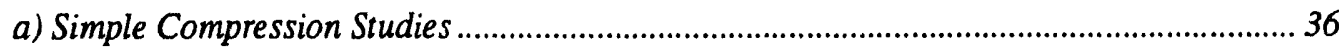

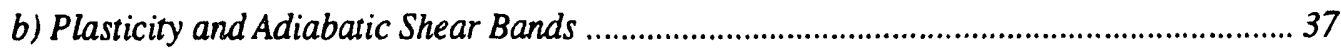

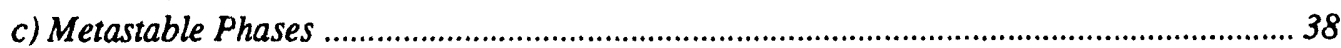

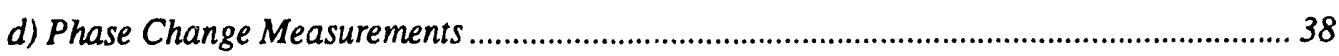

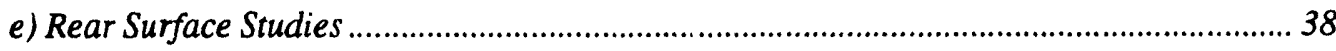

G) Hydrodynamics ....................................................................................................... 41

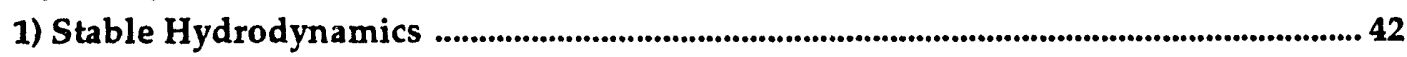

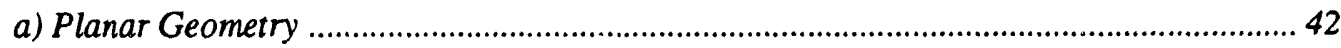

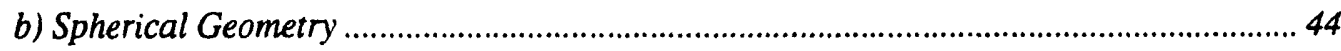

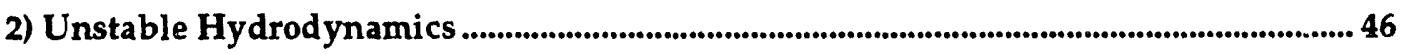

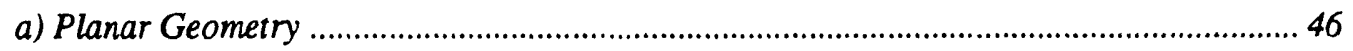

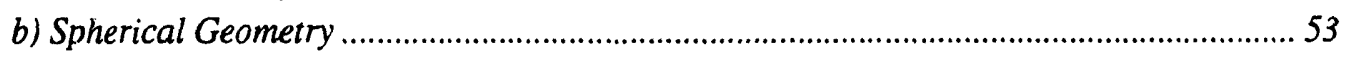


H) Absorption and Radiation Sources ...................................................................... 56

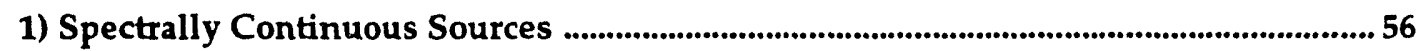

a) Conversion Efficiency of Au Burn-Through Foils ........................................................ 57

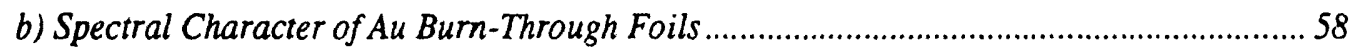

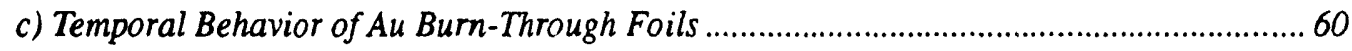

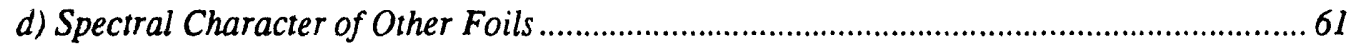

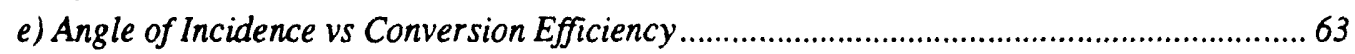

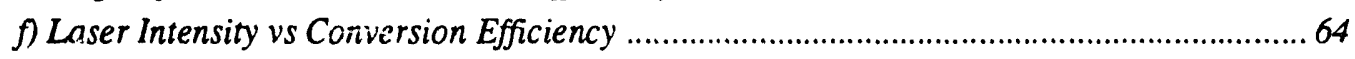

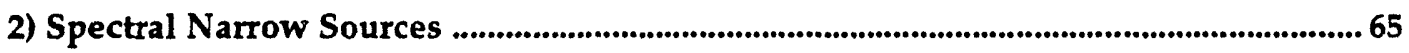

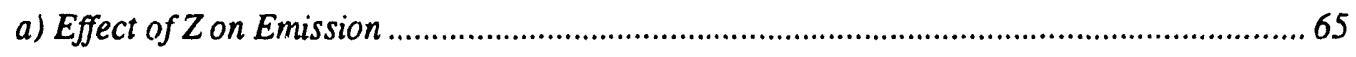

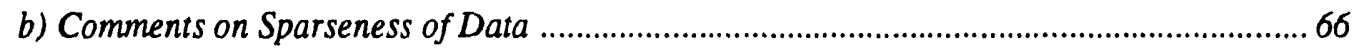

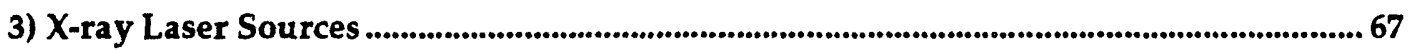

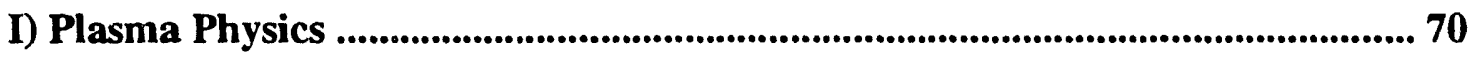

1) Interpenetrating Plasmas ..................................................................................................... 70

2) Plasma Streaming in Magnetic Fields ............................................................................... 72

3) Laser-Plasma Instabilities .............................................................................................................73

IV) Diagnostic Capabilities ...........................................................77

A) Overview of the Target Chamber Diagnostics ............................................... 78

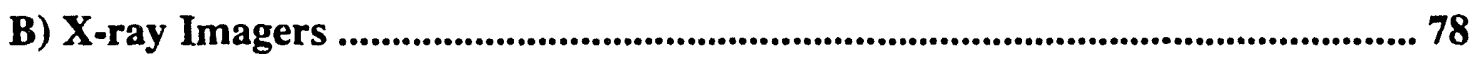

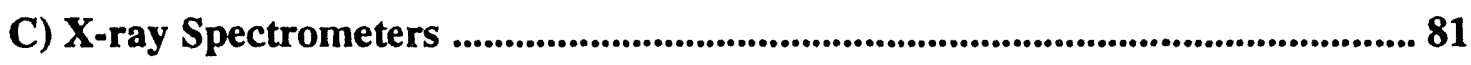

D) Optical Diagnostics ............................................................................................... 82

V) The Next Generation Facility - The NIF .........................................83

A) Introduction ...................................................................................................... 83

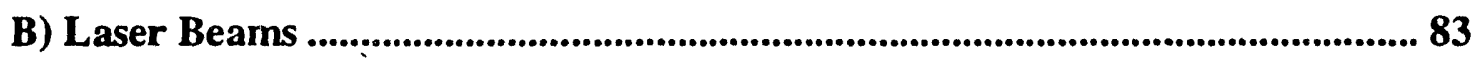

C) Beam Pointing, Aiming and Synchronicity .......................................................... 84

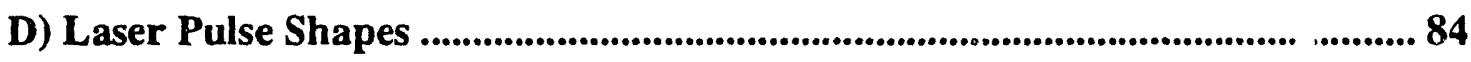

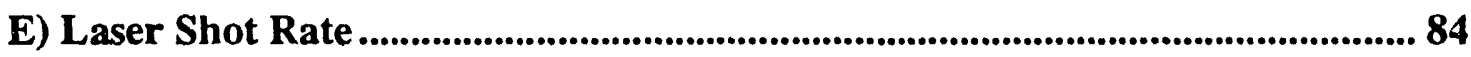

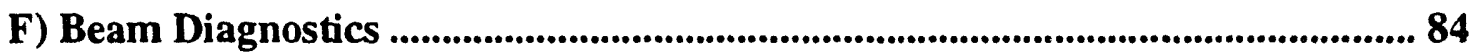

References ..............................................................................................85 85 


\section{List of Figures}

III.A-1) A schematic view of a hohlraum

II.A-2) A schematic view of some different hohlraums .........................................................................5

II.A-3) Radiation temperature vs in-out laser power ...........................................................................6 6

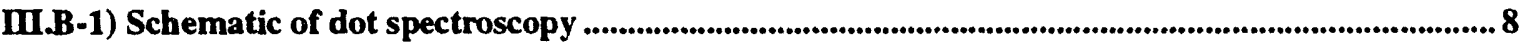

III.B-2) An example of the spectrum of lines of holmium .........................................................................8 8

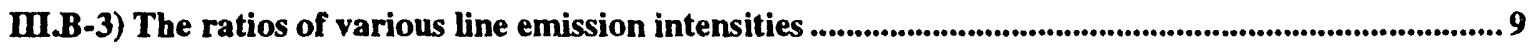

II.C-1) Schematic of a spectroscopy experiment ..................................................................................... 10

II.C-2) Temperature measurements derived from an experiment ..................................................... 11

III.C-3) The ratio of the $\mathrm{Cr}$ to Ti He-like 1s2-1s3p intensities ......................................................... 11

II.C-4) A spectrum of the $\mathrm{Cr}$ and $\mathrm{Ti}$ produced by irradiating a large bag of gas ................................ 12

II.D.1-1) Schematic of the radiative flow experiment ....................................................................13

II.D.1-2) A streak camera record of the backlight passing through a radiation flow package........... 14

II.D.2-1) Schematic of setup chosen to study non-LTE phenomena .....................................................17

II.D.2-2) Spectrum from the absorption of untamped BN .......................................................................... 18

II.D.2-3) The experimental results for the absorption of a Au backlight ............................................ 19

III.E.2-1) Experimental setup for plasma imaging using the $x$-ray laser as a backlight .....................21

III.E.2-2) X-ray laser backlit image of accelerated foil ...................................................................... 22

II.E.2-3) Moiré deflectogram of laser-irradiated CH target ...................................................................23

III.E.2-4) Schematic drawing of LLNL $x$-ray microscope, showing main components .......................23

II.E.2-5) Image of resolution test pattern obtained with $x$-ray laser microscope ..................................24

III.E.2-6) X-ray microscope images of rat sperm nuclei ................................................................25

III.F.1.a-1) Direct laser-driven shock experiment schematic .....................................................................27

III.F.1.a-2) Direct laser-driven shock experiment image .....................................................................28

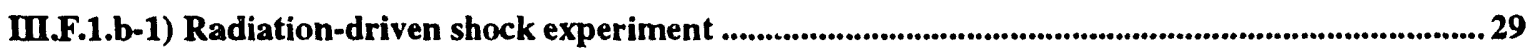

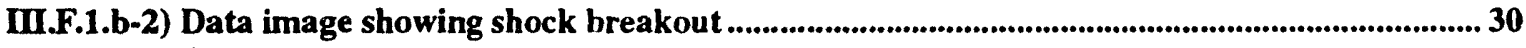

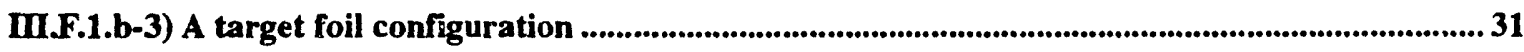

III.F.1.c-1) Overview of the arrangement for radiation-driven shock experiments ............................. 32

III.F.2-1) Schematic of the point projection spectroscopy for opacity measurements ............................. 33

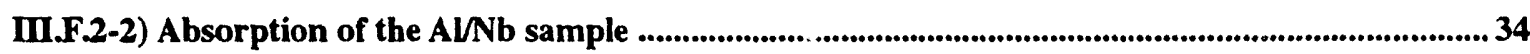

III.F.3-1) A schematic of the compression of a crystal using a laser-induced shock ............................36

III.F.3-2) A schematic of the rear surface shock breakout experiment .................................................. 39

III.F.3-3) Data from the rear surface of a material that has a shock transiting .................................40

III.F.3-4) Traces showing the spectra from the probed shocked rear surface ....................................41

III.G.1.a-1) Schematic of experiments to provide a test bed for hydrodynamic simulations ............... 43 
III.G.1.a-2) An example of the data obtained from the experimental test bed 43

III.G.1.b-1) 12-frame $x$-ray gated imager, called the WAX ................................................................. 44

III.G.1.b-2) Typical implosion sequence using the WAX diagnostic .....................................................45

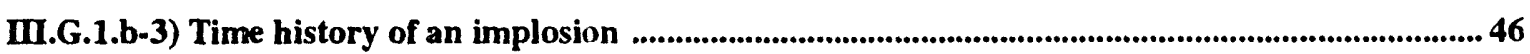

III.G.2.a-1) Schematic of the experimental setup .........................................................................47

III.G.2.a-2) The face-on image of the backlight source ......................................................................48

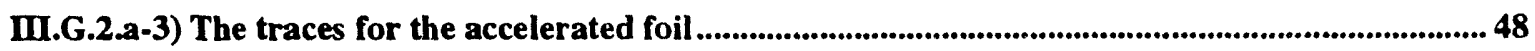

III.G.2.a-4) A schematic of the point projection spectroscopy technique .............................................50

III.G.2.a-5) A radiograph of a sulfinated plastic ablator . . .......................................................51

II.G.2.a-6) Av.jal distribution of mass areal densities .................................................................52

III.G.2.b-1) The machined "bumpy" ball ...........................................................................................5 53

III.G.2.b-2) Expanded view of an implosion image taken using the WAX .........................................5 54

III.G.2.b-3) Measured trace quantifying the limb brightening ..........................................................55

III.G.2.b-4) Streak camera records of spectra from microsphere ......................................................55

III.G.2.b-5) Streak camera records of spectra from a microsphere ...................................................55

III.H.1 a-1) Total x-ray emission measured as conversion efficiency vs target thickness ..................... 57

m.H.1.a-2) The measurement of the transmitted laser light ................................................................... 58

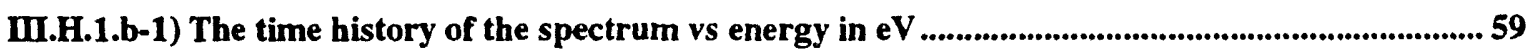

III.H.1.b-2) The spectrum of the Au M-bands .......................................................................................5 59

III.H.1.c-1) The temporal history of the spectrally integrated emission ............................................60

III.H.1.d-1) The spectral character of various elements from $Z=56$ to 63 ........................................ 61

III.H.1.d-2) Absolutely measured spectra emitted by a laser irradiated target ....................................62

III.H.1.d-3) Absolute conversion efficiency of laser-irradiated target ....................................................63

III.H.1.e-1) The effect of angle of incidence of the laser on $x$-ray conversion efficiency .................... 64

II.H.2.a-1) The conversion efficiency of various discrete transitions ...................................................... 66

III.H.3-1) Schematic drawing illustrating the double-pulse irradiation technique ..............................67

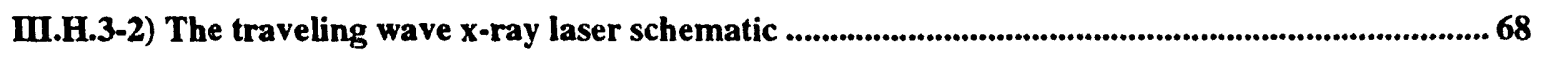

III.H.3-3) X-ray line emission in 3rd and 4th order with traveling wave ...........................................68

III.H.3-4) Time history of the emission from hard $x$-ray ..................................................................... 69

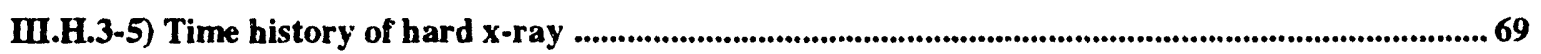

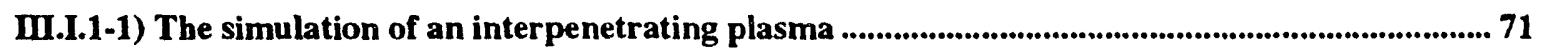

III.I.1-2) A schematic and an $x$-ray image of a colliding plasma experiment ...................................... 71

III.I.2-1) An optical image of a plasma streaming into a magnetic field ............................................... 72

III.I.3-1) A contour plot of the intensity of the Raman-scattered light vs wavelength and time .......... 74

III.I.3-2) Spectra for the back-reflected light due to SBS ...........................................................75

II.I.3-3) An image of laser-induced filamentation .........................................................................76

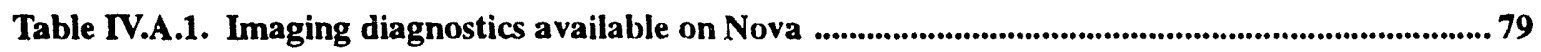




\section{I) Preamble}

The purpose of this document is to present to a general technically sophisticated audience the many possible technical areas that can be explored on a high-energy laser. The nature of a document of this kind implies that a certain level of detail will be lost. We accept this, but feel nonetheless that the advantages of presenting the many areas of active interest will be informative and a service as a starting point for further discussions.

Moreover, since a document of this kind must rely on numerous individuals providing information we would like to make an acknowledgment of these contributions here. The difficult work of scientists from the other institutions in the US should be recognized and we take this opportunity to thank all those at the Naval Research Laboratory (NRL), the Laboratory for Laser Energetics (LLE) and Los Alamos National Laboratory (LANL) for the many discussions and contributions that have been made to the generation of much of the work reported.

We also would like to acknowledge those that helped directly with the present document and will do this section by section-note that those individuals not at LLNL have their affiliated institutions listed. Definition of Existing Facility-J. Colvin of LANL, J. D. Kilkenny, R. W. Lee; Atomic Physics-J. R. Seely of NRL; Plasma Spectroscopy-R. W. Lee, C. A. Back, A. Osterheld, B. K. Young, B. MacGowan, W. Hsing of LANL; Radiative Transfer LTE Radiation Flow-R. Ward, T. Perry; Non-LTE Radiative Transfer-R. W. Lee, J. Moreno, J. A. Koch; Studying X-ray Lasers-J. Nilsen; Development of X-ray Lasers as Probes-L. Da Silva, B. MacGowan; Material Properties Equation of State-N. Holmes, R. C. Cauble; Directly Driven High Pressure Shocks-R. C. Cauble, L. Da Silva; Indirectly Driven Colliding Foil Experiments-R. C. Cauble, T. Perry; Opacity-T. Perry; Strength of Material-J. Wark \& N. Woolsey of Oxford University; Hydrodynamics Stable Planar Geometry-R. Klein, T. Perry; Stable spherical geometry-O. L. Landen, B. Hammel; Unstable planar geometry-B. Remington, G. Dimonte, J. D. Kilkenny; Unstable spherical geometry-O. L. Landen, B. A. Hammel; Backlights \& Radiative Sources-Continuous spectral sources-C. A. Back, R. W. Lee; Spectrally narrow sources-D. L. Matthems, J. Molitoris; X-ray laser sources-L. Da Silva; Plasma Physics Interpenetrating PlasmasP. Rambo, C. A. Back; Plasma Streaming in Magnetic Fields-T. A. Peyser; Laser-Plasma Instabilities-J. Moody, B. MacGowan; Diagnostic Capabilities-J. D. Kilkenny; Definition of Next-Generation Laser-C. Clower, G. Tietbohl, J. Colvin of LANL, J. D. Kilkenny.

Finally, we would like to thank J. Azevedo, J. Olivera and S. Jennings for all their help in putting this together. 


\section{II) Definition of the Existing Facility}

\section{A) Introduction}

The following definition of an existing high-energy laser facility is based on the Nova Laser facility at LLNL. Although this is currently the largest facility, it is generically similar to the other facilities currently extant. Note that the definition of the nextgeneration laser which will be contained in Section $V$ is being developed to a large degree with the assistance of the LLNL Laser Science program and these are individuals from which most of the facility information has been derived.

\section{B) Laser Beams}

The Nova facility has ten separate beam lines, each of which is roughly equivalent. The laser is based on the master oscillator power amplifier (MOPA) Nd:YLF configuration where the initial seed laser is generated in a desired temporally shaped pulse. The amplification occurs in a long chain of amplifiers separated by spatial filtering pinholes in increasingly larger diameter sections. The $1 \omega_{0}(1.053 \mu \mathrm{m})$ wavelength beam arrives at the target chamber with approximately 10 kilojoules of energy in a beam diameter of $74 \mathrm{~cm}$; this assumes a pulse that is temporally a 1-ns-square pulse. That is, the pulse rises in $100 \mathrm{ps}$ to a uniform intensity and persists for $1 \mathrm{~ns}$ until it falls in $100 \mathrm{ps}$. The beam is then either frequency doubled using a KDP crystal to a $2 \omega_{0}$ (green) or tripled, to a $3 \omega_{0}$ (blue) beam for final focusing down into the target chamber. The conversion efficiency is approximately $60 \%$, yielding $~ 4000$ joules of energy in the beam to be focused into the target chamber. The ten beams are balanced to produce $\leq 5 \% \mathrm{rms}$ deviation in energy.

The $1 \omega_{0}$ beam is of high spatial uniformity; however, the non-linearity of the up conversion to $2 \omega_{0}$ or $3 \omega_{0}$ amplifies any amplitude modulation in the beam. To smooth the beam , a major consideration for directly irradiating a surface where one desires uniformity, several techniques can be used. First, one can introduce a randomness in the phases of the beam with a random phase plate. This creates an effectively smoother intensity distr.bution by overlapping many small beamlets of random phase created from the large-aperture beam. Second, one can split the beam up into several pieces using wedges which allow an effectively flat intensity distribution over the focal spot. Third, one can introduce a band width on the normally monochromatic beam; this will, when combined with a diffraction grating, be used to move the focal spot, resulting in a temporally smoother intensity.

The limiting factor in the firing of the beam is the cooling-down time for the large disk amplifiers. This limits a particular laser beamline from firing more than once every hour. However, one must note that the complexity of the diagnostic setup and alignment of the target can, and often is, the limiting factor in the turn-around time of the experiments. 


\section{C) Beam Pointing, Aiming and Synchronicity}

The beam as it enters the chamber is focused into the target chamber center using an $\mathrm{f} / 4.3$ lens that will produce a minimum spot size of $150 \mu \mathrm{m}$ in diameter. The other options at the present time for the beam are the insertion of $f / 8$ lenses. This is a rather new option.

The accuracy of the pointing is $\pm 30 \mu \mathrm{m}$ rms with a maximum displacement of $2 \mathrm{~cm}$ of the beam centerline from target chamber center. The beams can be sent to three different target areas, a one-beam chamber, a two-beam chamber with the beams coming in opposing ports and a ten-beam chamber. In the ten-beam chamber the beams come into the chamber in two clusters of five beams entering on opposite sides of the midplane. The five beamlines are placed in a five-sided symmetry and come into the chamber in a cone with a $50^{\circ}$ half angle. The synchronicity is \pm 20 ps at target chamber center.

\section{D) Laser Pulse Shapes}

Pulse durations can vary from 100 to 600 ps Gaussians to 1- to 5-ns-square pulses. There is also flexible pulse shaping capability, so that the energy can be delivered in 2- to 3-ns shaped pulses with $>10$ intensity contrast between peak and foot of the pulse. Two of the beams can be delayed, independently of each other, by up to $25 \mathrm{~ns}$ after the main beam. This last capability has been found useful for providing a source of backlighter $\mathrm{x}$-rays.

\section{E) Laser Shot Rate}

The laser currently fires at a rate of approximately 115 shots per month. This is consistent with the fact that the laser fires about seven shots per day utilizing a twoshift system. The week has four scheduled experimental days and one day maintenance.

\section{F) Beam Diagnostics}

The diagnostics that are available of the beam performance are as follows. First, the pulse shape is monitored in the master oscillator for temporal signature and energy. Next the propagated beam can be monitored for the total 1- $w_{0}$ energy and the temporal variation of the 1- $\omega_{0}$ energy. Further, images can be made of the near and far field of the $1-\omega_{0}$ beam.

The up-converted beam, in either $2 \omega_{0}$ or $3 \omega_{0}$, can be monitored in the chamber for absolute as well as temporal intensity. Further, images of the beam front can be produced in the far or near field. 


\section{III) Experimental Capabilities and Techniques}

\section{A) Introduction and Overview}

To illustrate the experimental capabilities of high-energy lasers we will briefly discuss recent experiments that are challenging the current limits of our ability and older experimental techniques that have become well established. In this way we provide information on capability and give some indications of the experiments that are in progress.

We note that the repetition rate of the experiments is relatively low when compared to table-top laboratory experimental facilities. On the other hand, the system has, for a high-energy-density production facility, a fairly rapid turnaround time. The inherent problem of using facilities to obtain both high temperatures and high densities tends to lead to various high levels of destruction. In this particular class of facilities the highenergy laser has a high duty cycle. However, even when compared to the table-top facilities, there are benefits which can help offset the relatively low duty cycle.

The most obvious mitigating factor in support of the high-energy laser for research work is the fact that the diagnostics complement that can be brought to bear on a single experiment is very large. Second, and pedantically, is the fact that the high-energy lasers can perform experiments that cannot be performed at any other type of facility. Thus, the high-energy laser can be seen as a distinct breed of facility that permits novel regimes to be investigated, but which allows these investigations to occur in real time.

As an overview of the capabilities of a laser system of this sort we provide the following. Firstly, a unique feature of the system is the hohlraum. A hohlraum is, in the jargon of the inertial confinement fusion (ICF) program, a high-Z-usually $\mathrm{Au}-\mathrm{cylin}$ der with end caps. The laser enters the cylinder through the ends and is focused on the interior walls in an annular pattern. The laser light is converted to $x$-rays by laser-matter plasma creation. The size of a typical hohlraum is $2400 \mu \mathrm{m}$ long with an $800-\mu \mathrm{m}$ radius. The radiation temperature equivalent, found by measuring the emission from the hohlraum and equating it to sT4 is $\sim 200 \mathrm{eV}$.

The temperature and the size and scale of the hohlraums can, of course, be changed to accommodate experimental needs. See Fig. III.A-1 for an example. The design of a hohlraum can affect the radiation temperature and conditions of the targets placed inside.

In Fig. III.A-2, three different types of hohlraums are shown, each of which has a different utility. Note that the radiation temperature is changed by the hohlraum scale. In Fig. III.A-3 the scaling of the radiation temperature measured vs incident laser energies is shown. In the figure there is also a simple fit to the data using empirically determined parameters, the conversion efficiency of the laser energy to $x$-ray energy, $h$, and the amount of loss of the laser energy due to heating of the walls, $\alpha$; these are determined to be 0.7 and 0.8 respectively for the scale- 1 geometry. The two areas that play a role are the area of the walls, $A_{w}$, and the area of the holes in the wall, $A_{h}$. 


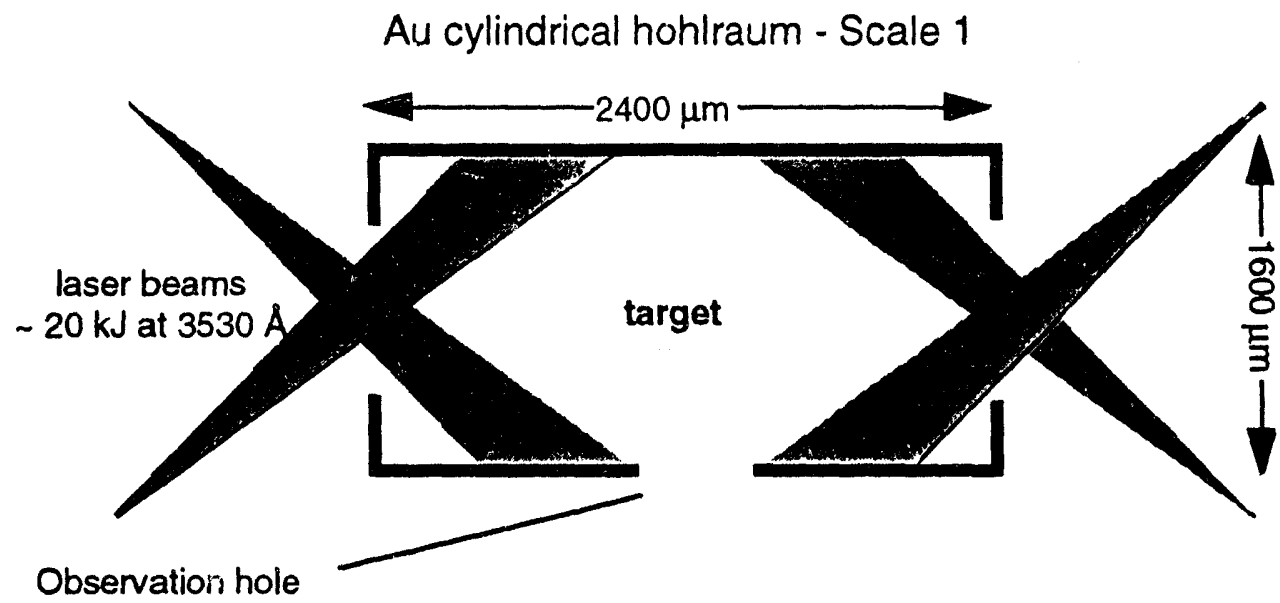

Laser Pulse Shapes

- Up to $28 \mathrm{~kJ}$ in 10 beams

- Square: $1 \mathrm{~ns}$, flat top, $100 \mathrm{ps}$ rise

- Shaped: 3 to 1 contrast, 2.2 ns
Targets

- Microshperes of gas filled

- Tamped opacity samples

- Cylinders for convergent hydro

III.A-1) A schematic view of a hohlraum. The one shown here is the nominal hohlraum, called a scale 1; it generates a radiation temperature of $\mathbf{2 0 0} \mathrm{eV}$.

\section{Scale 3}

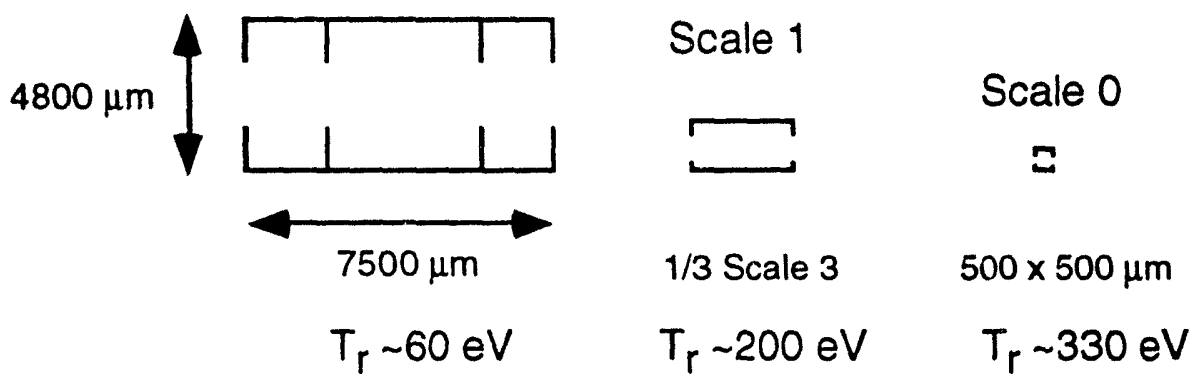

III.A-2) A schematic view of some different hohlraums which are used to create a radiation environment for various purposes. Numerous configurations exist for different tasks.

The ten beams typically deliver $20-30 \mathrm{~kJ}$ of $0.35-\mu \mathrm{m}$ light into a cylindrical gold hohlraum that is $2.5 \mathrm{~mm}$ long with a diameter of $1.6 \mathrm{~mm}$, producing a uniform quasithermal source of $\mathrm{x}$-rays. $\mathrm{X}$-ray drive produces high ablation pressures that are spatially very smooth. The radiation can be used to drive capsule implosions, drive planar 


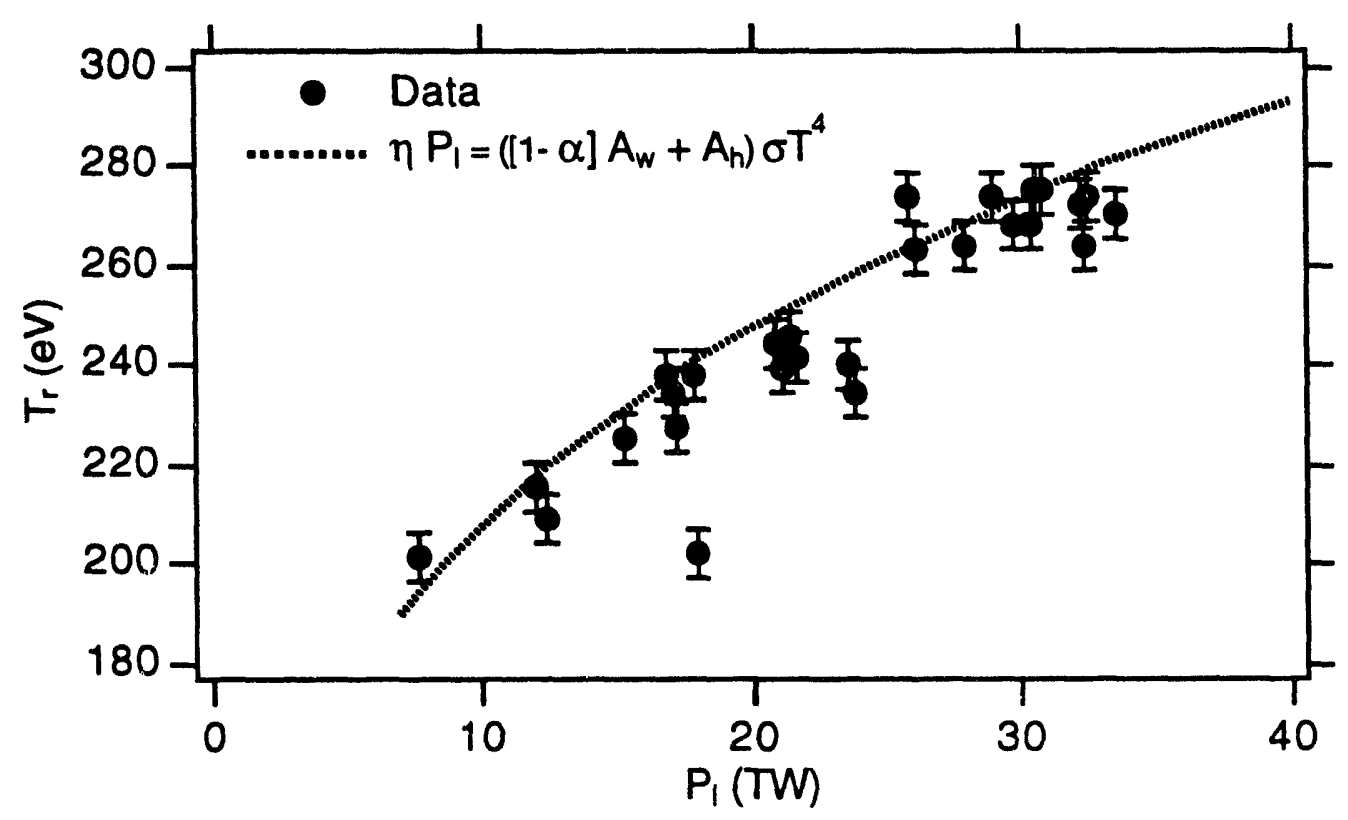

III.A-3) Radiation temperature vs in-out laser power. Temperature is derived from equating the measured energy output to $\sigma \mathrm{T}^{4}$. The data points are indicated, and an empirical fit is shown as the dashed line.

shocks in material samples for hydrodynamics, provide a local thermodynamic equilibrium (LTE) environment for opacity studies, and provide drive for equation-of-state studies. The shock pressure created by radiation drive is a strong function of the temperature:

$$
\mathrm{P}(\mathrm{Mbar})=2.3 \times 10^{4} \mathrm{~T}^{3.5}(\mathrm{keV}) .
$$

For example, approximately 100-Mbar shocks can be driven in plastic with 200-eV drive.

The laser is also used for direct illumination of targets. The direct illumination of low-density targets, for example foams or gases, can produce plasmas with an electron temperature of a few $\mathrm{keV}$. Ablation pressures generated in directly driven targets are:

$$
\mathrm{P}(\text { Mbar })=12\left[\mathrm{I}\left(10^{14} \mathrm{~W} \mathrm{~cm}^{-2}\right) / \lambda(\mu \mathrm{m})\right]^{2 / 3} .
$$

Thus, for laser light with a wavelength of $\lambda=0.53 \mu \mathrm{m}$, (frequency-doubled light) and an intensity of $\mathrm{I}=2 \times 10^{14} \mathrm{~W} / \mathrm{cm}^{-2}$, the ablation pressure generated is about $10 \mathrm{Mbar}$. Directly driven shocks can be made reasonably planar over about $1 \mathrm{~mm}$ by smoothing the beam profiles with random phase plates and SSD.

The areas that we will address are: plasma physics, hydrodynamics, material properties, atomic physics, radiative properties, implosion dynamics, radiation transfer, $\mathbf{x}$-ray laser studies, and absorption and radiative source production. Many of the areas that are employed below to provide some structure are, of course, not precise and 
overlap must occur. As a simple example, the material property "opacity" is important in radiative transfer, but for historic reasons it is mcluded in the Materials Properties section.

\section{B) Atomic Physics}

The study of the atomic physics of highly ionized atoms has come into the modern era with the availability in the late 1960 s of reasonably high-energy lasers. The production mechanism for obtaining ionized material is straight-forward: irradiate a solid surface of the element of interest with a laser at intensities greater than the surface melting point. This technique has produced spectra and line identifications of numerous highly ionized species. The method has been improved with the use of a localized dot of material in a matrix of a lower $\mathrm{Z}$ material to help isolate the element of interest to a finite column that will move outward along the laser axis from the solid surface. This is schematically illustrated in Fig. III.B-1.1

The dot spectroscopy technique has been widely used to improve spectral resolution, which can be compromised by source broadening, and permit spectral information from spatially resolving instrument to isolate regions of the plasma in which there are small temperature and density variations.

Along this line we mention two results. First, we have the work of Seely and others performing classical line identification on higher $Z$ elements. In this work a survey soft $x$-ray spectrometer with wide spectral coverage is used to look in the XUV region. Results of this technique have been used to obtain, for example, the configuration interaction and level crossing data relevant to the $3 \mathrm{~d}^{10} 414 \mathrm{l}^{\prime}$ levels in highly ionized zinc-like atoms with atomic numbers from 40 to $80 .^{2}$ These experiments are used to provide difficult-to-obtain data.

Second, the identification of highly stripped ions in laser-produced plasma can lead to interesting diagnostic possibilities. In experiment where the XUV spectrum can be both space and spectrally resolved has led to the inference of the density-sensitive emission line ratio from holmium $X X X I X .{ }^{3}$ In Fig. III.B-2 we show an intensity-vswavelength plot of the lines of interest at one spatial position. Various ratios of these emission lines, assuming a temperature of $1780 \mathrm{eV}$ and an optical thin plasma, form a reasonable diagnostic. In Fig. III.B-3 the ratios of various ratios are shown vs electron density.

The final comment on the spectroscopy of the laser-produced plasma is the interest in the structures called unresolved transition arrays, UTAs, which are spectral features composed of myriad line transitions from complex ions. These structures provide a wealth of information on the plasma formation and atomic physics of complex atoms. ${ }^{4}$ 
Front view

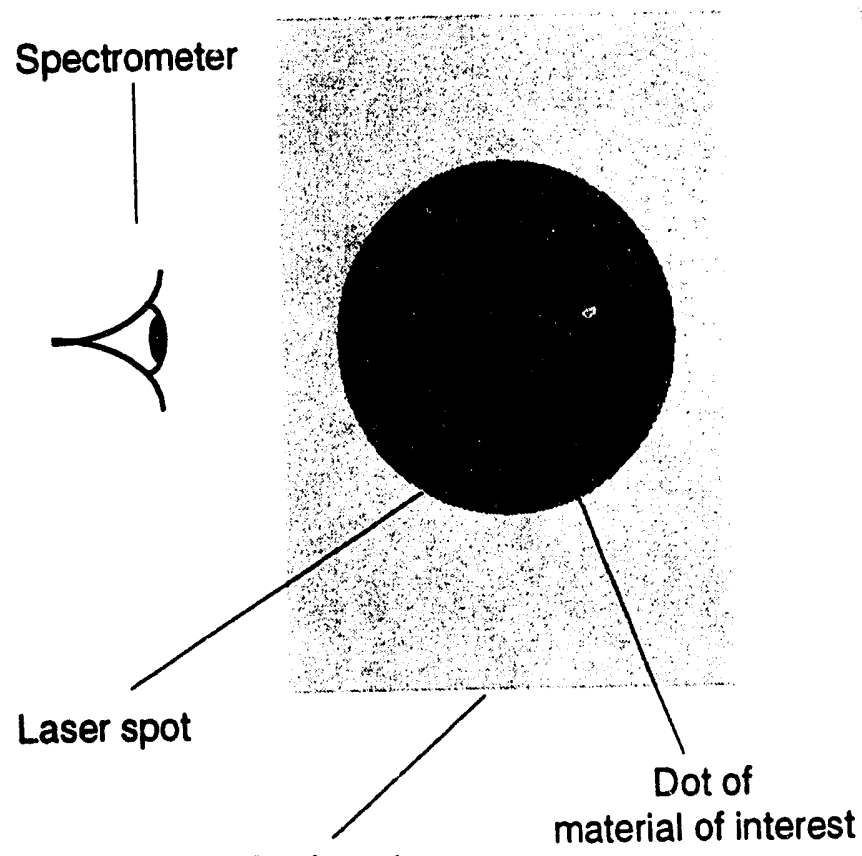

Top view

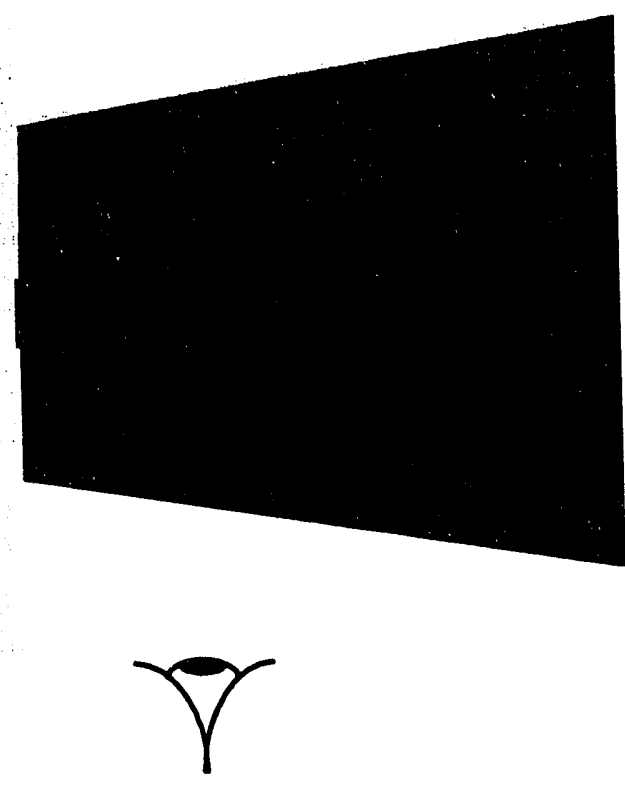

low-Z substrate

III.B-1) Schematic of dot spectroscopy. The dot of the material of interest is placed on, or slightly beneath, a target that will not interfere spectroscopically. When the surface is irradiated, the dot is confined, reducing the spatial variations possible.

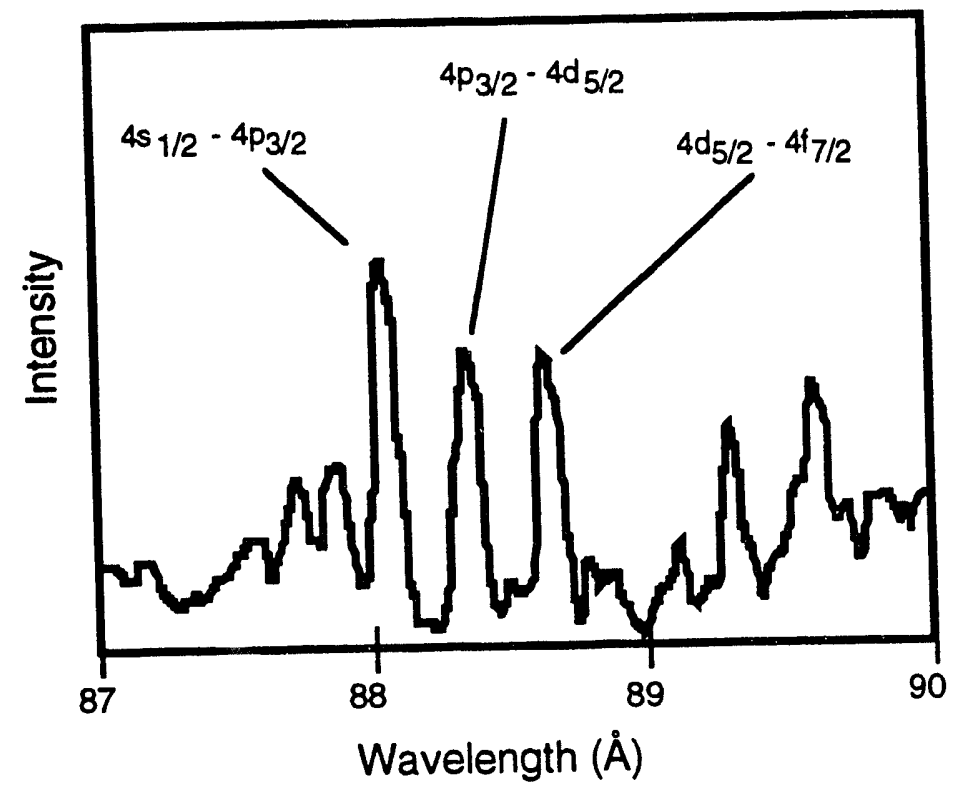

III.B-2) An example of the spectrum of lines of holmium XXXIX $n=4$ to 4 lines at one spatial position. The lines used in the diagnostic are indicates. 


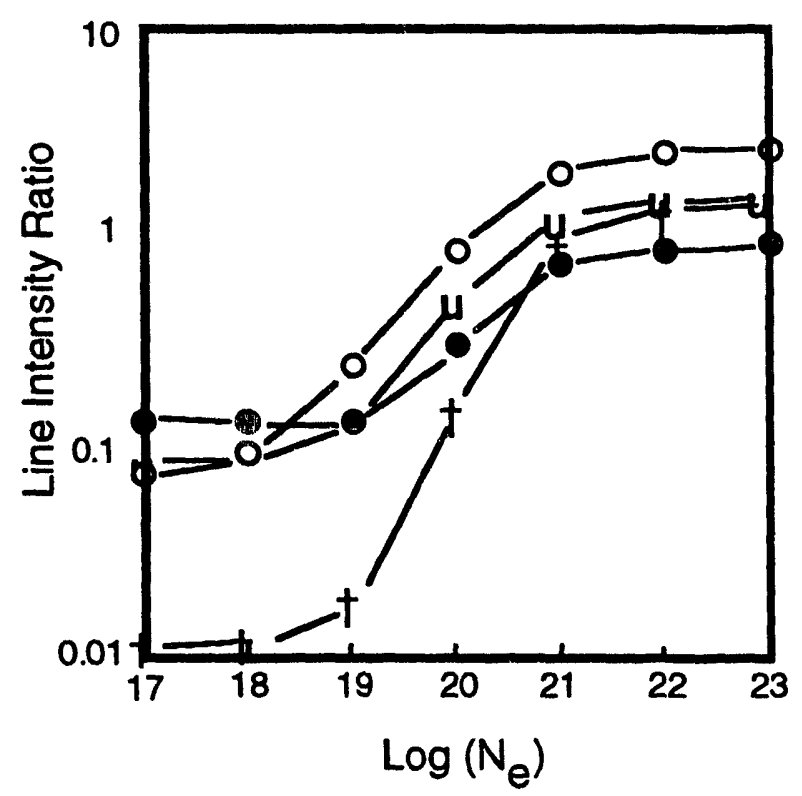

II.B-3) The ratios of various line emission intensities: $u={ }^{2} P_{3 / 2}-{ }^{2} D_{5 / 2}$ to ${ }^{2} S_{1 / 2}-{ }^{2} P_{3 / 2} ; \quad+={ }^{2} D_{5 / 2}-{ }^{2} F_{7 / 2}$ to ${ }^{2} S_{1 / 2}-{ }^{2} P_{3 / 2}$ $0={ }^{2} P_{1 / 2}-{ }^{2} D_{3 / 2}$ to ${ }^{2} S_{1 / 2}-{ }^{2} P_{3 / 2} ; \quad \cdot={ }^{2} D_{5 / 2}-{ }^{2} F_{7 / 2}$ to ${ }^{2} P_{3 / 2}-{ }^{2} D_{5 / 2}$

\section{C) Plasma Spectroscopy}

The plasma produced by directly irradiating targets gives rise to plasma states that are well outside of the defined limits to satisfy the conditions for LTE in the plasma. The time dependence of the heating source, together with the fact that the plasma expands rapidly during heating by the laser pulse, indicate that these plasmas must be studied using non-LTE techniques. This, in turn, means that atomic models coupled to non-LTE rate equations must be employed to predict the populations in the plasma, and thus are necessary for interpretation of the spectral character in general.

The further complication that the plasmas are spatially varying indicates that the hydrodynamics must also be modeled for a fuller understanding of the plasma. To understand laser-produced plasmas much effort has been expended to develop a diagnostic complement that will allow one to obtain data independent of plasma simulations. These methods include the development of the instruments, which will be discussed in Section IV, as well as the development of plasma spectroscopic diagnostics. Many of these diagnostics are complementary to those developed for astrophysical, atmospheric or other laboratory plasmas.

One of the methods that has found great success is the previously mentioned dot spectroscopy technique. Results of this technique have been perfected so that the temporal, spatial, and spectral information is obtained on a single shot with independent measures of, for example, the electron density. A schematic of such a setup is shown in Fig. III.C $-1 .{ }^{5}$ The results for the electron temperature measurement that uses the slope of 


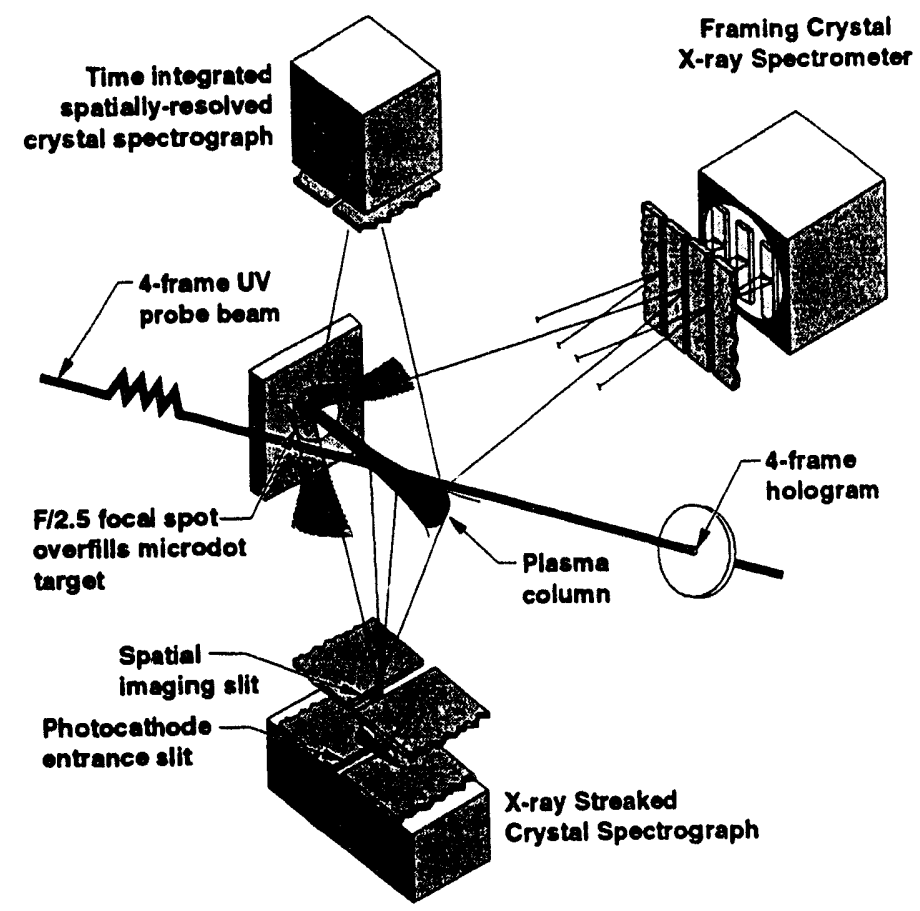

III.C-1) Schematic of a spectroscopy experiment that provides time, space and spectral information. In this experiment a holographic interferometer is also used to provide an independent measure of the plasma electron density.

the bound-free emission continuum of the hydrogenic species is shown in Fig. III.C-2. This represents the type of detailed spectroscopy that one can perform on these plasmas even though they are rapidly evolving.

This brings us to an important point concerning the laser irradiation of a surface. The plasma that is formed during the laser-matter interaction will be dense and warm at the surface, becoming tenuous and hot as the matter moves away from the target. Therefore, defining the temporal and spatial history of the material is a challenging feature of these experiments. This, as one might expect, gives rise to a field of study of its own.

One rather elegant advance was to use a series of concentric dots of differing elements, bull's-eye fashion, to isolate the spatial information in the direction perpendicular to the laser axis, and then to use a gated spectrometer that provided spatial resolution along the laser axis. In this form of the experiment the non-LTE character of the plasma can be analyzed, as well as used for simple line identification.

The methods developed for use on the laser-produced plasmas include the standard line intensity ratio techniques, the use of spectral line broadening, and most recently, a novel, but simple, extension of the line ratios technique to the use of two different elements. ${ }^{6}$ The idea can be illustrated in Fig. III.C-3 where the ratios of the He-like $1 \mathrm{~s} 2-1 \mathrm{~s} 3 \mathrm{p}$ transitions is shown from titanium to chromium in the same plasma conditions. In Fig. III.C-4 we show the results of an experiment on a long scale length plasma 


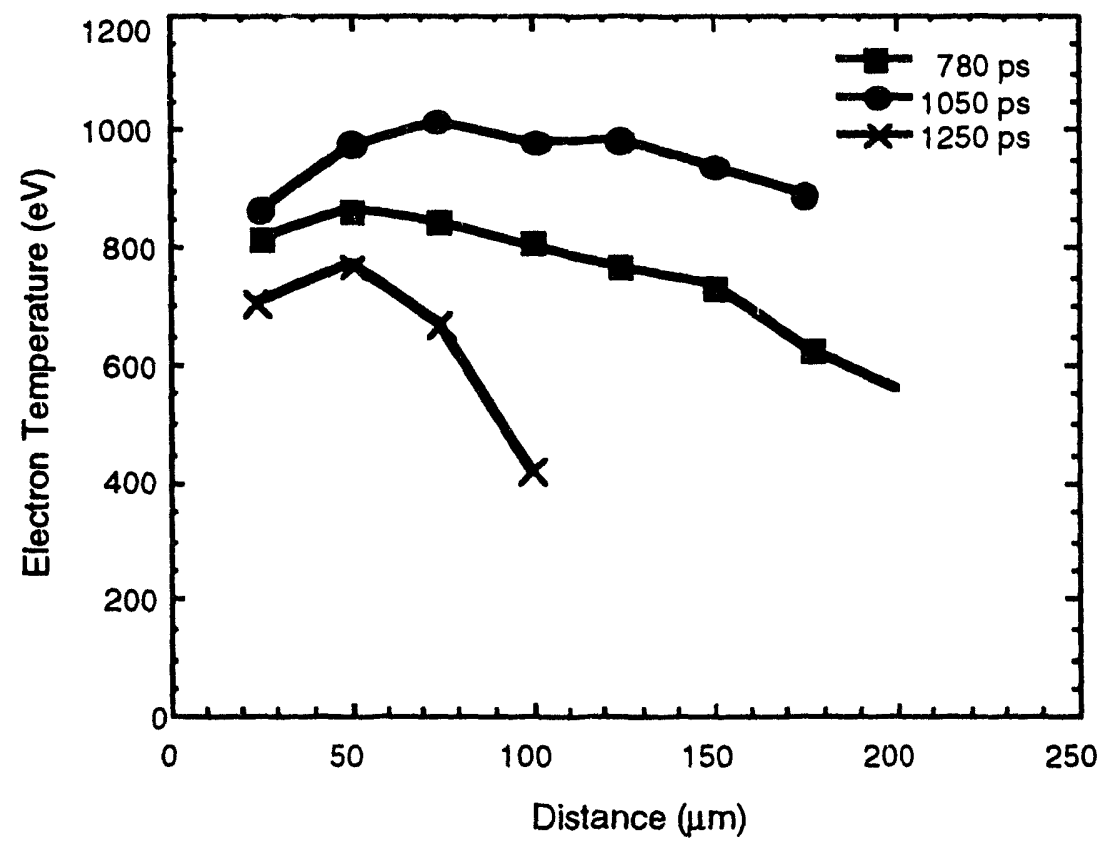

III.C-2) Temperature measurements derived from an experiment of the type shown schematically in Fig. III.C-1. The temperature from the hydrogenic bound-free recombination continuum is measured for several frames on a spatially resolving spectrometer.

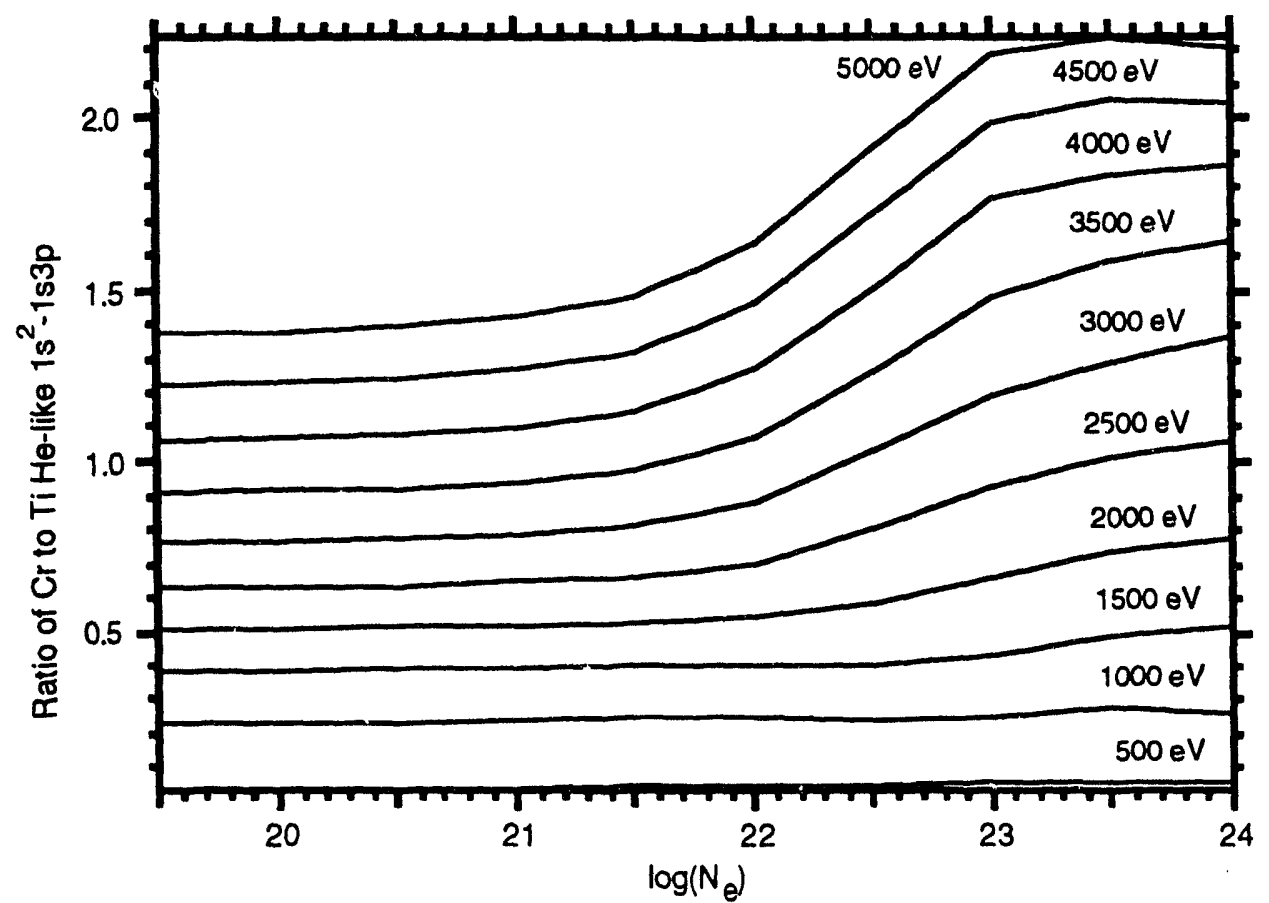

III.C-3) The ratio of the $\mathrm{Cr}$ to $\mathrm{Ti}$ He-like $1 \mathrm{~s}^{2}-1 \mathrm{~s} 3 \mathrm{p}$ intensities for a range of temperatures and densities. Note that each curve represents a single temperature. 


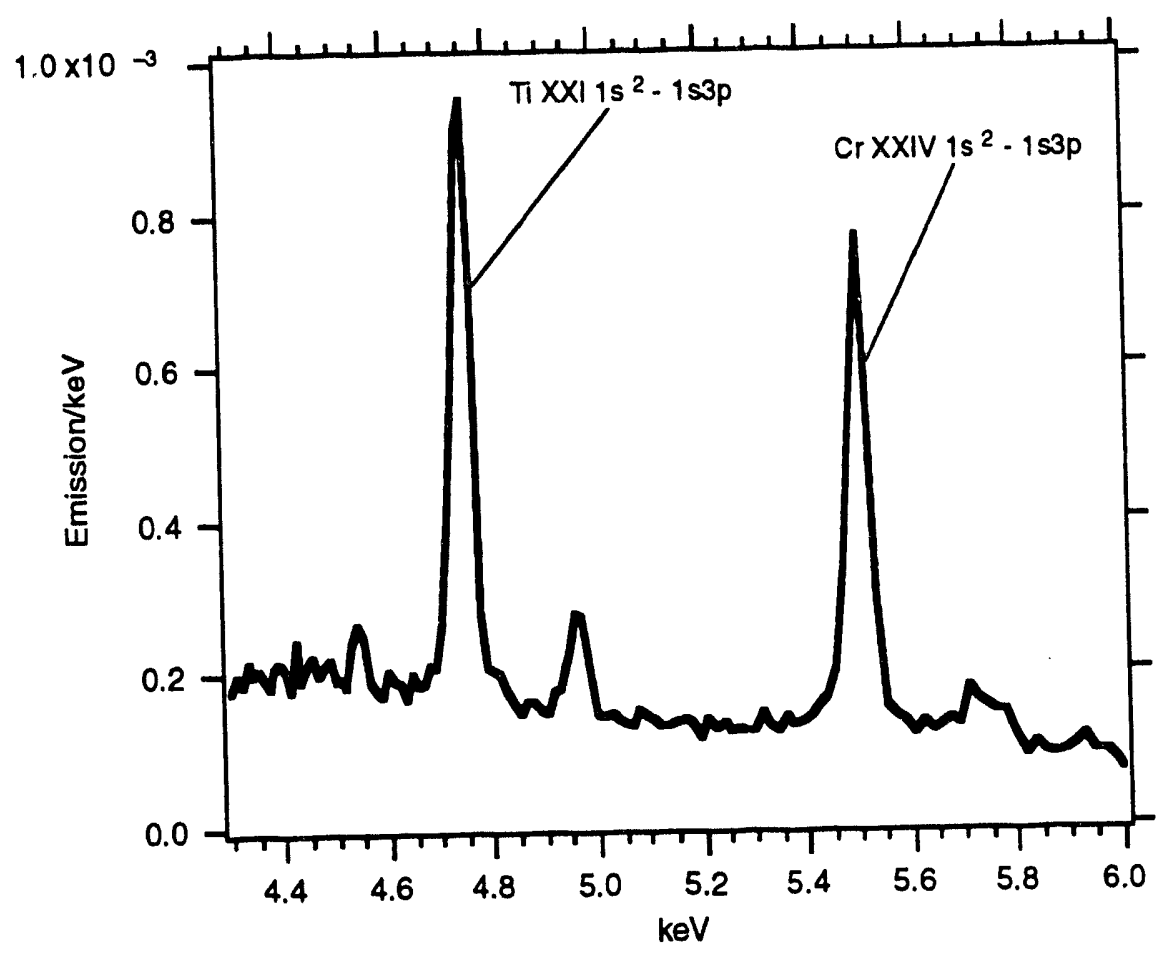

III.C-4) A spectrum of the $\mathrm{Cr}$ and Ti produced by irradiating a large bag of gas with a single 3-ns long pulse of $3600-\mathrm{J}$ energy. The $\mathrm{Cr}$ and $\mathrm{Ti}$ are on fibers introduced into a bag of $n$-pentane, yielding an $N_{e}$ of $10^{21} \mathrm{~cm}^{-3}$. The line ratios indicate the $T_{e}$ is $-3 \mathrm{keV}$. The electron density of the gas is relatively undisturbed, due to the small amount of hydrody. namic motion, and thus this temperature can be determined.

created by irradiating a bag of gas which is underdense. That is, when fully ionized, the electron density $\left(\mathrm{N}_{e}\right)$ is less than the critical density $\left(\mathrm{N}_{c}\right)$ for the laser frequency used. The laser heats the gas to a few kilovolts, and the $\mathrm{Cr} / \mathrm{Ti}$ line ratios are a good diagnostic of this temperature. From $\mathrm{Cr}$ - and $\mathrm{Ti}$ - coated fibers introduced into a bag of n-pentane having an $\mathrm{Ne}$ of $10^{21} \mathrm{~cm}^{-3}$, the line ratio in Fig. III.C-4 indicates that the $\mathrm{T}_{\mathrm{e}}$ is $\sim 3 \mathrm{keV} .^{7}$

In terms of novel diagnostics measurement techniques have been developed that employ line shapes, ${ }^{8}$ inner shell absorption techniques, ${ }^{9}$ quasi-steady-state models, ${ }^{10}$ as well as $x$-ray laser probes. ${ }^{11}$ Some these will be discussed in later sections.

\section{D) Radiative Transfer}

One of the unique capabilities of the high-energy lasers is that one can reach sufficient energy density, albeit for short times, to permit the study of radiation transfer as a topic in its own right. Since the transport of radiation plays an integral part in stellar interiors, as well as in ICF implosions, this has become an area of some interest. There are clearly two generic regimes. In the first regime, when the transfer of radiation occurs in material that satisfies the conditions for LTE, the transfer is studied as a flow of radiation through the material. In these experiments one is interested in measuring the 
flow characteristics as these are represented by quantities such as the Rosseland Opacities and described by the radiation diffusion approximation.

In the second regime one is dealing with plasmas that do not satisfy the conditions for LTE and the demands become somewhat more complex. Whereas in LTE we can use the Saha-Boltzmann equation or some related statistical method to obtain populations, in non-LTE cases we require atomic models of some sophistication that demand rates for all the processes of importance. Further, the radiation diffusion equation is no longer useful and one must make recourse to the solution of the detailed radiative transfer equation that, in general, must be solved self-consistently with the rate equations.

\section{1) LTE Radiation Flow}

Currently there is a series of experiments to study the transfer of heat by $\mathrm{x}$-rays through targets of varying types. The basic experimental geometry is shown in Fig. III.D.1-1a. X-rays from a Nova hohlraum are incident on one end of a millimeterscale tube. In Fig. III.D.1-1b the various targets used in the radiation flow experiments

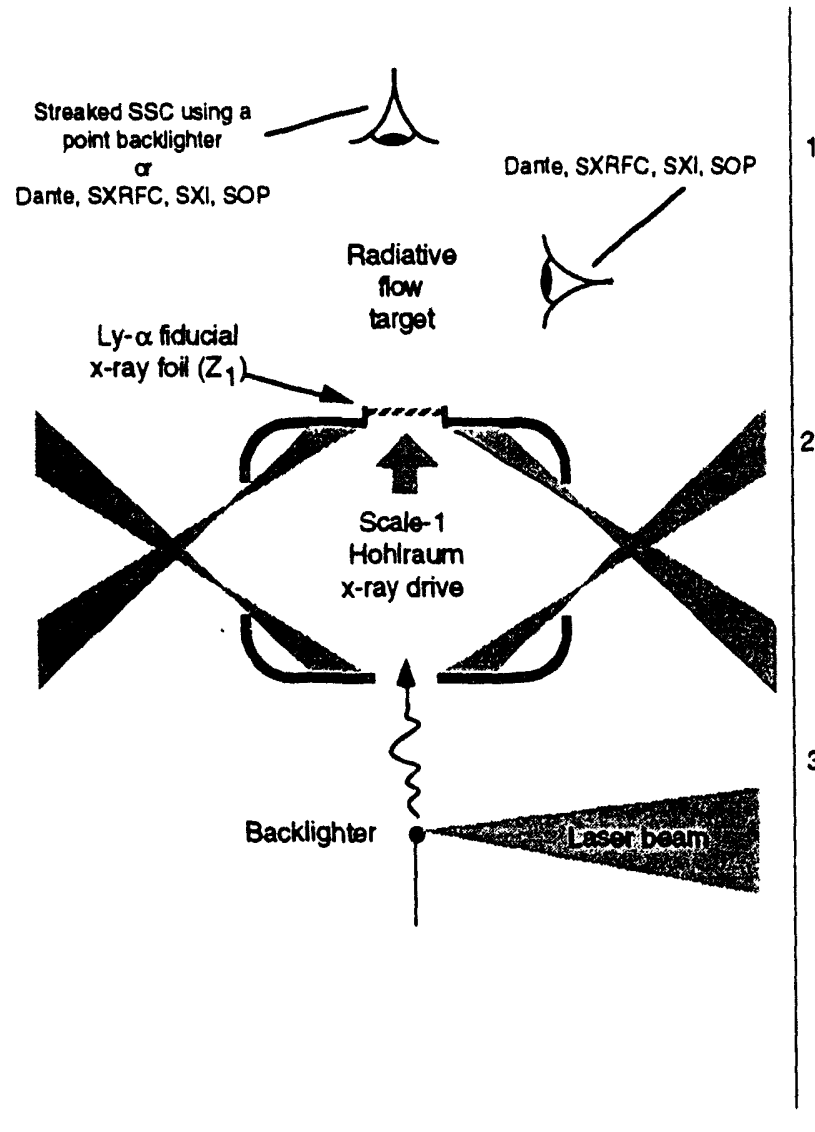

a) Experimental schematic

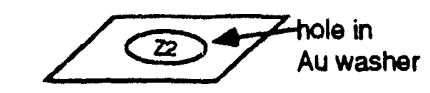

1) Vacuum between $Z_{1}$ and $Z_{2}$

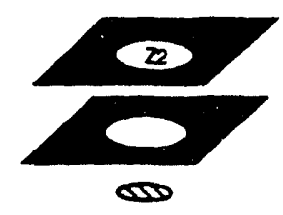

2) Occluding Au obstructions

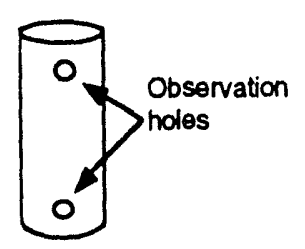

3) Empty or foam-filled tube

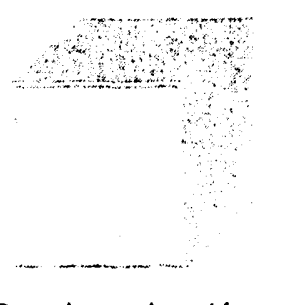

4) Doped or undoped foam

b) Radiative flow targets

III.D.1-1) Schematic of the radiative flow experiment. The numerous possible experimental packages are shown separately. The instrument names can be located in Section IV. 
are shown. Since the hohlraums represent a feature of these, and many other, experiments, the hohlraum is shown in Fig. III.A.-1 and -2.

The $x$-ray drive for these experiments was created by focusing eight of the ten laser beams at Nova into a gold hohlraum. The other two Nova beams were used for creating the backlighter illumination. Behind the radiation flow target is a tiny fiber of samarium that is heated by laser light to produce a broad-band $x$-ray backlighting spectrum at photon energies from around 1 to $2 \mathrm{keV}$. On the other end of the tube is an $x$-ray crystal coupled to an $\mathrm{x}$-ray streak camera.

The tube contains a series of thin fiducial foils that register the transfer of heat by allowing one to observe the absorption spectra of $\mathrm{Ka}$ transitions in the foils. That is, as the fiducial foils heat they go through several different ionization stages. Each ion stage absorbs different frequencies from the $x$-ray backlighting spectrum. The streak camera record is able to give the ionization history of each of the fiducial foils in the tube. From this, the time history of the temperature of each foil can be inferred.12

Fig. III.D.1-2 presents an example of the streak camera data record for the flow through a tube. Both 1D burn-through foils and more complicated 2D geometries are being studied. The initial $x$-ray driving flux has been well characterized in terms of its time-, angle-, and frequency-dependence. Heat-transfer experiments are also being performed using a 2D x-ray framing camera to study the longitudinal distribution of heat in both unobstructed and baffled straight cylindrical geometries.

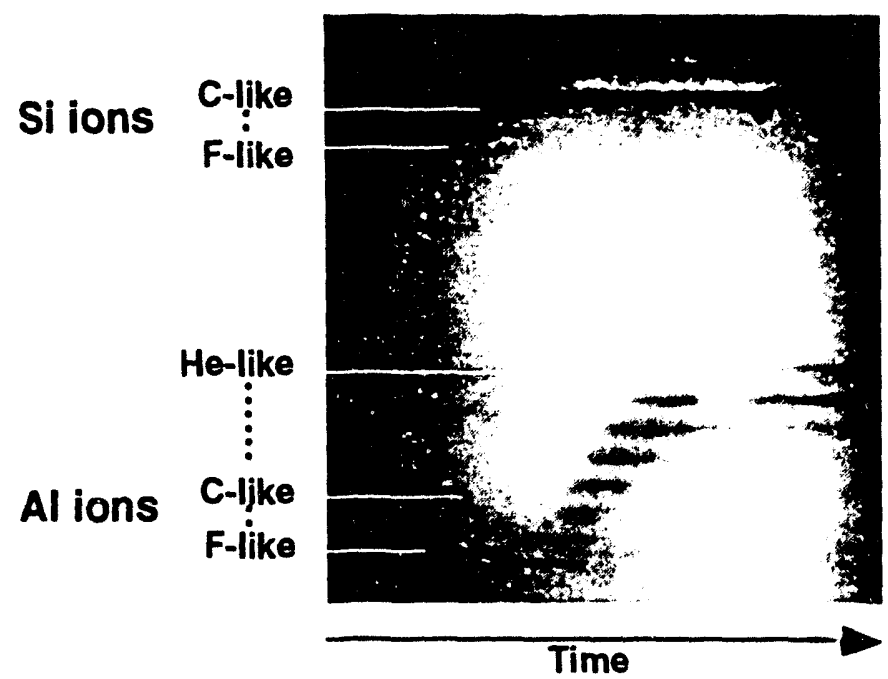

III.D.1-2) A streak camera record of the backlight passing through a radiation flow package. Time runs vertically and the spectrum is horizontal. At early time the $\mathrm{Si} \mathrm{K} \alpha$ lines are seen, and as the radiation flows up the tube the image shows the $\mathrm{Al} \mathrm{K} \alpha$ lines rise up at late time. 
These initial experiments have also been extended using smaller hohlraums that create hotter radiation sources capable of driving longer and more complex 2D geometries. The radiative drive from these hotter sources have also been well-characterized.

\section{2) Non-LTE Radiative Transfer}

The interest in the generation of $x$-ray lasers led to a series of relatively recent advances in theory and experiment that have brought us to a position where detailed radiation hydrodynamics simulations are available and detailed kinetics model can be produced, with some effort. (It is important to note the central role of the astrophysicist in the development of these simulation capabilities. On the one hand astrophysicists were producing the methodology for doing non-LTE radiation transfer "correctly," but on the other hand, by far the largest part of the motivation for the experiments shown here was derived from interest in laboratory plasma sources.)

Thus, there are simulation codes that now provide a radiative-hydrodynamics capability for the analysis of non-LTE experiments. A description of the basics of these codes is not necessary here, but the fact remains that these codes exist and have been developed at great expense. The rigorous testing of the codes has essentially been confined to benchmark numerical problems where analytic solutions exist; however, no rigorous tests against controlled high-energy-density plasma experiments has been performed.

The first goal of the effort on non-LTE radiative transfer will be to develop a method to compare the results of radiative hydrodynamics simulations that goes as far as possible towards creating an environment in which detailed comparisons can be performed. That is, we will provide the initial conditions more accurately than anything previously attempted and then provide the results of the experiment. The hope is to provide the results in the form of absolute intensities so that the details of the simulations can be tested for the first time.

More specifically, there are three areas in which it is felt simulation capabilities should be tested. First, the accuracy of the atomic kinetics models will be measured insofar as the spectral information we will provide will be of high enough quality to check model details.

Second, the suitability of the kinetics models in the simulation can be tested. The ability to obtain experimental information on the evolution of the radiatively driven system will allow one to ascertain if the kinetics model is sufficient to simulate the plasma dynamics.

Third, and most difficult, the level of information that will be available may be sufficient to constrain the radiation transfer algorithms in these time-dependent multilevel systems. Note that the testing of the radiative transfer would require the generation of spectra that contain line transitions formed in a radiatively dominated regime. This is a very difficult regime to attain in the laboratory for the following reasons: A simple rule of thumb for radiation to dominate the collisions in the line formation 
process is that the $\varepsilon$ parameter, defined as the ratio of the collision decay to the radiative decay of a transition, be small compared to unity, and the optical depth of the transition be large. The $\varepsilon$ can be evaluated as

$$
\varepsilon \approx 10^{-4} \mathrm{Ne} \lambda^{3}
$$

where $\lambda$ is the transition wavelength in $\mathrm{cm}$ and $\mathrm{N}_{\mathrm{e}}$ is the electron density in $\mathrm{cm}^{-3}$. Here we assume the Gaunt factor is 0.2 and the plasma temperature is $100 \mathrm{eV}$. Next, the optical depth of a Doppler transition, $\tau$, can be approxim ated by assuming that the oscillator strength is $\approx 0.5$, the temperature is $\approx 100 \mathrm{eV}$ and the atomic number is $\approx 20$. Then

$$
\tau \approx 6.3 \times 10^{-10} \mathrm{~N}_{\mathrm{g}} \lambda l
$$

Here lis the plasma column length in $\mathrm{cm}$ and $\mathrm{N}_{\mathrm{g}}$ is the ground state number density. If we desire the line formation to work in a regime where the source function reaches the blackbody limit, so that the medium is effectively thick, one can relate the $\varepsilon$ to the $\tau$ by,

$$
S_{\max } / B \approx \varepsilon \tau \sqrt{\ln (\tau)} \text { (for Doppler line profiles) }
$$

Analysis indicates that, for example, a column length of $10 \mu \mathrm{m}$ of plasma with an electron density of $10^{20} \mathrm{~cm}^{-3}$ and $10^{19} \mathrm{~cm}^{-3}$ ground state ions will yield for $100-\AA$ radiation an $\varepsilon \approx 0.01, \tau \approx 64.0$, and a maximum source function near the blackbody limit. These conditions are difficult to attain, but are possible in the type of experiments that are currently being performed. The main object of this work, the sfore, would be to develop the techniques to perform these measurements. Detailed studies of parameter regimes where $\varepsilon$ is much smaller and/or $\tau$ is much larger are important to the verification of the radiation transfer schemes; however, the goal of the present study is to create an experimental environment in which detailed studies can eventually be performed. But, first things first.

The progress so far on these experiments is as follows. First, an experimental configuration is now being tested. The considerations that led to this are:

- The use of radiation enclosures, i.e., hohlraums, is not necessary for the production of non-LTE experiments. The enclosure provides nothing of interest to the experimental design, and in fact complicates the measurement process to the point of rendering the experimental plan all but unattainable.

- The use of direct drive experiments, although of great interest to the community of experimentalists as a fertile source of ideas and developments, produces a plasma that is heated by mechanisms not relevant to the testing of the non-LTE radiative hydrodynamics simulations. 
- The dimensionality of the experimental system must be reduced to the minimum so that we can provide as nearly as possible an ideal non-LTE system. This is achieved by using a low- $Z$ tamping layer to contain the material of interest.

- Since the temperatures one can reach are well below the kilovolt range, the spectral measurements should be made in the sub-kilovolt range. This will require an effort to develop XUV instruments.

Given these considerations the experiment can be defined, and a schematic of the experiment as it is being fielded is shown in Fig. III.D.2-1. The experimental plan is now going forward on three levels. First, we have developed a full understanding of the radiation heating source. The source, which arises from a Au burn-through foil, has been measured in time, spectrum and angle (for more information see the section on Absorption and Radiation Source). ${ }^{13}$

Second, we have developed the methods to perform the absorption spectroscopy of the heated samples. Figure III.D.2-2 shows an example of the spectrum from an untamped BN target. The figure shows a comparison of the theoretical absorption spectrum and experiment for this case. The BN sample is untamped; consequently the effects due to the vacuum/matter interface will be present.

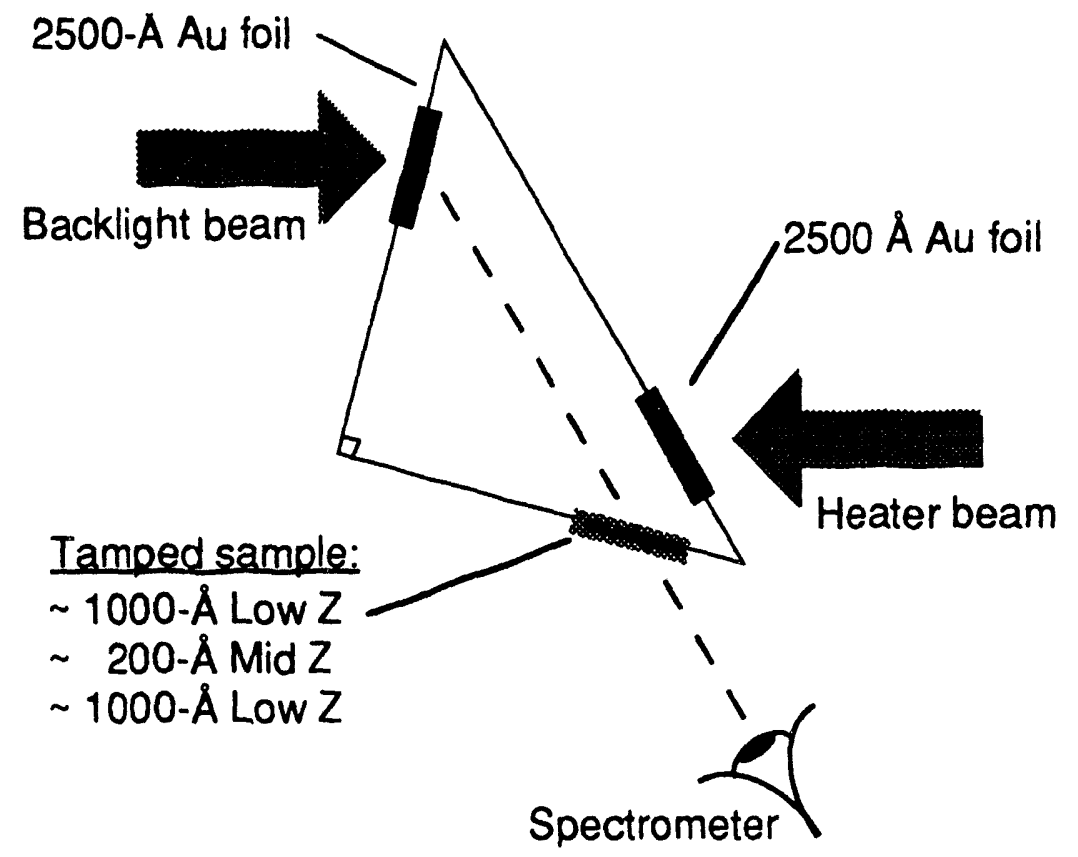

III.D.2-1) Schematic of setup chosen to study non-LTE phenomena. Heating is achieved by using the flux from the rear side of a Au burn-through foil. The sample in this case is tamped, and the backlight can be delayed in time for the heating. 


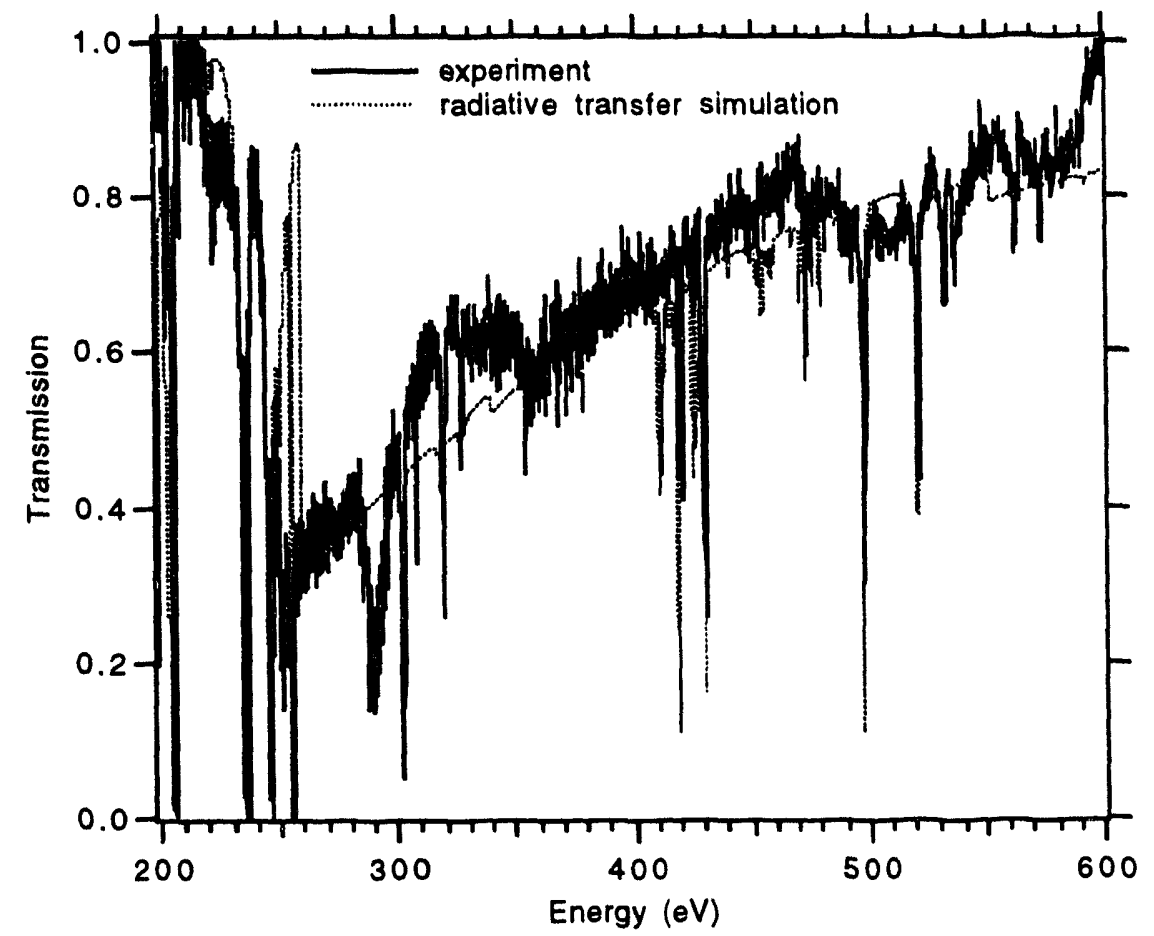

III.D.2-2) Spectrum from the absorption of untamped BN. The experiment is the solid line; two predictions of the spectra are also shown. The dashed line is the prediction of the non-LTE radiative transfer code Altair. Note that this sample should not come too far out of LTE, and comparisons indicating this have been performed.

The tamped sample spectroscopy and the relevant characterization of the material with small gradients is the third step. In Fig. III.D.2-3 we see the first attempts at measuring a Stark-broadened absorption line profile to ascertain $\mathrm{N}_{\mathrm{e}}$ as a function of time. The density can be determined as a function of time by using a long duration backlight (i.e., absorption source) with an XUV streak camera. Then the time-dependent width of the absorption line yields a time-dependent density.

The development of in situ temperature diagnostics and further density diagnostics is being evaluated.

\section{E) X-ray Lasers}

There are three distinct paths of research for $x$-ray laser studies. First, there is research into the details of the mechanisms that have made the first viable laboratory $x$-ray laser possible. Second, there is an effort to use the currently developed lasers as a source to study other phenomena. Third, there is the continuing work to develop the $\mathbf{x}$-ray laser as a light source for future applications. Although these three avenues are not completely distinct it is simpler to present it in this way. The first two will be discussed here, the third will be discussed in the section on Absorption and Radiation Sources. 


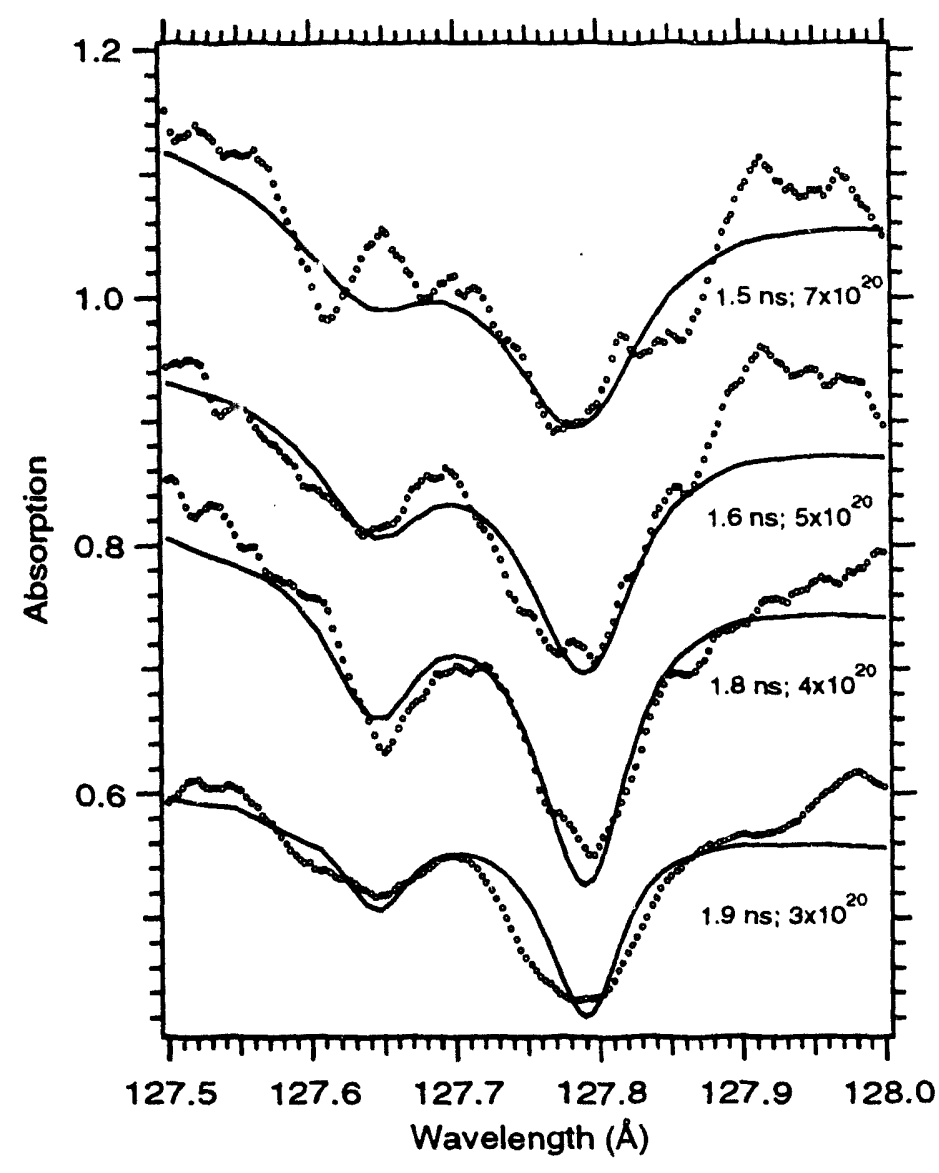

III.D.2-3) The experimental results for the absorption of a Au backlight through a CH-tamped $\mathrm{CF}_{2}$ sample. The measurement shows ii-like fluorine measured along with the theoretical prediction for the line shape. The densities inferred from this are shown in the figure.

\section{1) Studying $X$-ray Lasers}

Since the first demonstration of soft $x$-ray lasing using the collisional excitation mechanism in Ne-like Se, many other Ne-like ions, ranging from $\mathrm{Cu}, \mathrm{Z}=29$ to $\mathrm{Ag}$, $Z=47$, have been made to lase. ${ }^{14}$ However, attempts to produce lower $Z$ Ne-like $x$-ray lasers had been unsuccessful. In the effort to develop a table-top $x$-ray laser that would require smaller high-energy laser drivers than Nova and could be used for applications such as biological imaging, nonlinear optics, holography, etc., a prepulse technique has been developed. This technique has been used successfully to produce lasing in many lower $\mathrm{Z}$ Ne-like ions such as $\mathrm{Ti}, \mathrm{Z}=22 ; \mathrm{Cr}, \mathrm{Z}=24 ; \mathrm{Fe}, \mathrm{Z}=26$; and $\mathrm{N}, \mathrm{Z}=28 .{ }^{15} \mathrm{The}$ use of this prepulse technique has opened up a new class of Ne-like $x$-ray lasers for investigation.

In studying the Ne-like lasers it was noticed that the ions with odd $Z$ do not lase as well as those with even $Z$. Since elements with odd $Z$ have a nuclear spin and a nuclear moment while those with even $Z$ tend to have no nuclear spin, one possible explanation for this anomalous behavior is that hyperfine splitting is playing an important role in the gain of the Ne-like laser lines for elements with odd Z. Using a high-resolution grazing incidence grating spectrometer we measured the line shape of the $\mathrm{J}=0$ to 1 , $3 \mathrm{p}-3 \mathrm{~s}$ transition in Ne-like $\mathrm{Nb}$ and $\mathrm{Zr}$ and observed a $28-\mathrm{mA}$ splitting between the two 
largest hyperfine components in the $\mathrm{Nb}, \mathrm{Z}=41$, line at $145.9 \AA$, in good agreement with theory. As predicted, no splitting was observed in $\mathrm{Zr}, \mathrm{Z}=40.16$ This $131-\mathrm{cm}^{-1}$ splitting is the largest hyperfine splitting ever observed and is also the shortest wavelength transition $(145.9 \AA$ ) and most highly ionized plasma ( 31 electrons) in which the hyperfine effect has been directly observed, with the exception of some very recent accelerator experiments in hydrogen-like bismuth done at GSI Darmstadt.

Further studies have been performed on the use of low-density foams as possible $x$-ray laser targets. Currently, $x$-ray laser experiments use either thin foils or thick slab targets at solid density, which are illuminated by Nova, heat, and expand to reach the low densities required for lasing. This hydrodynamic expansion introduces large electron density gradients in the plasma; these result in large refraction effects as the x-rays propagate down the laser axis.

If targets could be fabricated from uniform low-density materials at the density required for lasing (i.e., $<3 \mathrm{mg} / \mathrm{cm}^{2}$ ), then the beam propagation and ultimately the laser coherence could be improved. Successful observations have been made of lasing in $\mathrm{Zr}$ aerogel foam at $90 \mathrm{mg} / \mathrm{cm}^{3}$. At lower density Mo-doped agar foam at $3 \mathrm{mg} / \mathrm{cm}^{3}$ and Se-doped agar at $8 \mathrm{mg} / \mathrm{cm}^{3}$ have been tried without success. However, the aerogel foams have much smaller cell sizes (500 $\AA$ compared with 1-2 $\mu \mathrm{m}$ for agar) and look very promising if the density can be lowered.

Very recent experiments are using a traveling-wave geometry to produce shortpulse $x$-ray lasers for use in imaging experiments on ICF targets. The traveling wave geometry enables us to drive the x-ray laser targets with short pulses, $\sim 100 \mathrm{ps,} \mathrm{while}$ still using a long enough target, $\sim 3 \mathrm{~cm}$ or greater, to produce strong laser output. We have recently observed a hundred-fold contrast in Ne-like Ge x-ray lasers observed with and against the direction of the traveling wave. For more details see the section on Absorption and Radiation Sources.

\section{2) Development of $X$-ray Lasers As Probes}

Since the collisionally pumped soft $x$-ray lasers now operate over a wavelength range extending from 35 to $300 \AA$, and these sources have high peak brightness, one can now start to use them for $x$-ray imaging and plasma interferometry. The attraction of these light sources is extraordinary brightness. The brightness of these $x$-ray lasers was estimated to be equivalent of a Planckian of temperature of $40 \mathrm{keV} .17$

Since the collisionally pumped soft $x$-ray lasers now operate over a wavelength range extending from 35 to $300 \AA$, and these sources have high peak brightness, one can now start to use them for $x$-ray imaging and plasma interferometry. The attraction of these light sources is extraor inary brightness. The brightness of these $x$-ray lasers was estimated to be equivalent of a Planckian of temperature of $40 \mathrm{keV}$. Applications of the $x$-ray laser probe to long-scalelength plasmas using Moiré deflectometry and soft x-ray imaging can be demonstrated. The progress in the development of short-pulse $x$-ray lasers using a double-pulse irradiation technique that incorporates a traveling wave pump will be shown in the section on Absorption and Radiation Sources. 
The first of the $x$-ray laser probe applications is shown in Fig. III.E.2-1. Here a series of experiments has begun to characterize drive uniformity of thin-foil targets irradiated with laser beams that are smoothed with random phase plates. In this experiment $10-\mu \mathrm{m} \mathrm{CH}$ foils overcoated with $3 \mu \mathrm{m}$ of $\mathrm{Al}$ are irradiated at $1014 \mathrm{~W} / \mathrm{cm}^{2}$ with a 1 -nssquare, $0.53-\mu \mathrm{m}$-wavelength beam from the Nova laser. The focal spot distribution is effectively a flat-top distribution over a $700-\mu \mathrm{m}$ diameter region. The accelerated foil is backlit with $a \sim 150$-ps $x$-ray laser pulse from an exploded yttrium $x$-ray laser target. The $x$-ray laser operates at $155 \AA$ and has an output energy of $\sim 7 \mathrm{~mJ}$.

In Fig. III.E.2-2 we show the image as recorded on an $x$-ray CCD detector. The spatial resolution is estimated to be better than $2 \mu \mathrm{m}$. The $5-6 \mu \mathrm{m}$ filament structure observed on the rear surface is likely due to the speckle pattern produced by the random phase plates. This result clearly shows the merits of using $x$-ray lasers to image highdensity plasmas. Although direct imaging using an $x$-ray laser does not require several of the attributes of the $x$-ray laser (specifically, small source size, narrow divergence and narrow bandwidth) the high brightness allows one to neglect self-emission. The short duration of the x-ray laser is useful, however, in that it allows us to use an x-ray CCD as the detector, which has better spatial resolution $(27-\mu \mathrm{m}$ pixel), and higher dynamic range $(>1000)$, than existing gated microchannel plate systems.

Using a similar experimental setup, Moiré deflectometry experiments have been performed to measure the electron density profile in a laser irradiated $\mathrm{CH}$ plasma. The only difference between the imaging and Moire experiments is the addition of two

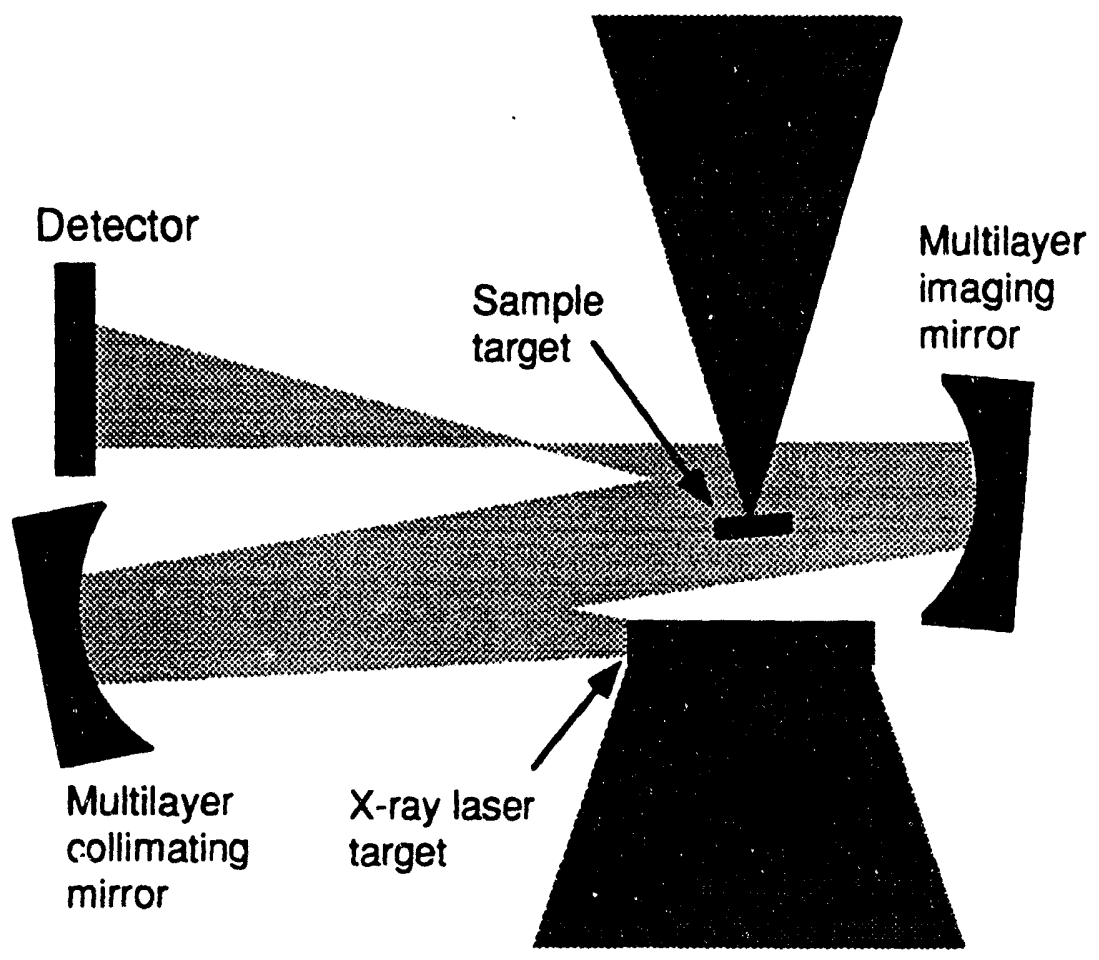

III.E.2-1) Experimental setup for plasma imaging using the $x$-ray laser as a backlight. 


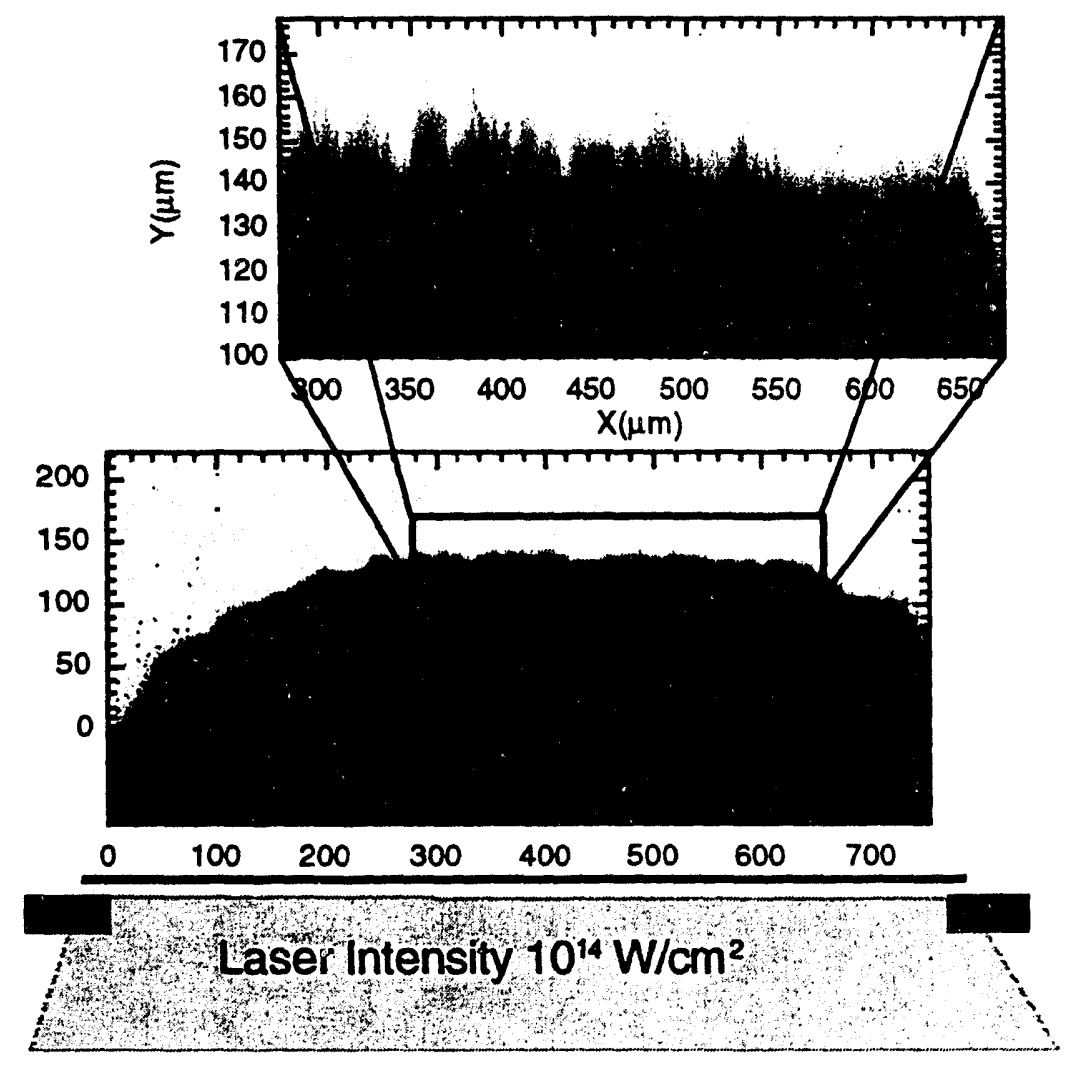

III.E.2-2) X-ray laser backlit image of accelerated foil. Foil is $10 \mu \mathrm{m}$ $\mathrm{CH} / 3 \mu \mathrm{m} \mathrm{Al}$ and irradiated, with beam smoothed with random phase plates. Top picture is central region of foil, and shows nonuniformities at the rear surface with dimensions characteristic of the speckle pattern.

Ronchi rulings directly in front of the detector. The rulings have a period of $10 \mu \mathrm{m}$ and a relative rotation of 4 degrees to give a fringe spacing of $142 \mu \mathrm{m}$ in the image plane-i.e., $14.2 \mu \mathrm{m}$ in the object plane. The rulings were separated by $19.35 \mathrm{~mm}$ to give a sensitivity of $5.2 \mathrm{Mrad} /$ fringe-shift at the object plane.

Figure III.E.2-3 shows the measured deflectogram obtained of a $\mathrm{CH}$ target irradiated at $2.5 \times 10^{13} \mathrm{~W} / \mathrm{cm}^{2}$. The laser pulse was a 1-ns square pulse at a wavelength of $0.53 \mu \mathrm{m}$ and focused to a 3-mm dia. spot. The image shows the expected unperturbed Moiré pattern far away from the surface, but shows clear deflections closer to the irradiated side of the target. Analysis of the fringe pattern indicates peak electron densities of $2 \times 10^{21} \mathrm{~cm}^{-3}$ near the surface. This corresponds to a peak line-integrated density of $6 \times 10^{20} \mathrm{~cm}^{-2}$, a factor of 30 higher than is accessible using conventional UV interferometry at $\lambda \sim 2650 \AA$.

The second application employs the $x$-ray laser in an imaging microscope. In Fig. III.E.2-4. a schematic of the $x$-ray imaging microscope is shown. X-rays from a tantalum nickel-like collisionally pumped $x$-ray laser operating at $44.83 \AA$ are collected and focused onto a specimen. The $x$-ray laser was generated by irradiating a $3.5-\mathrm{cm}$ long, 2000- $\AA$ thick plastic foil coated with $900 \AA$ of tantalum with two cylindrically focused laser beams operating at a wavelength of $0.53 \mu \mathrm{m} .{ }^{18}$ The heated foil explodes to 


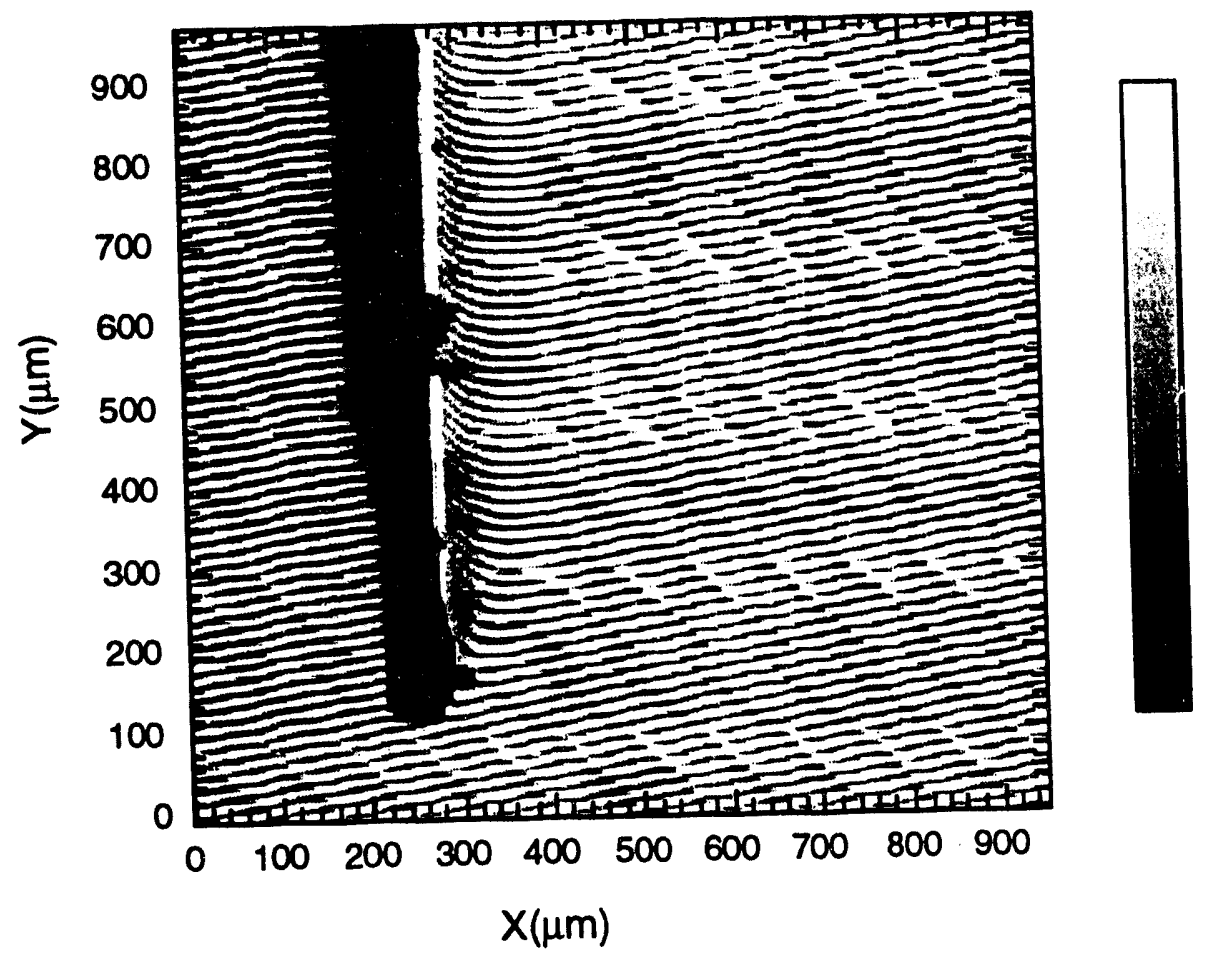

III.E.2-3) Moiré deflectogram of laser-irradiated CH target. Laser beam is incident from the right.

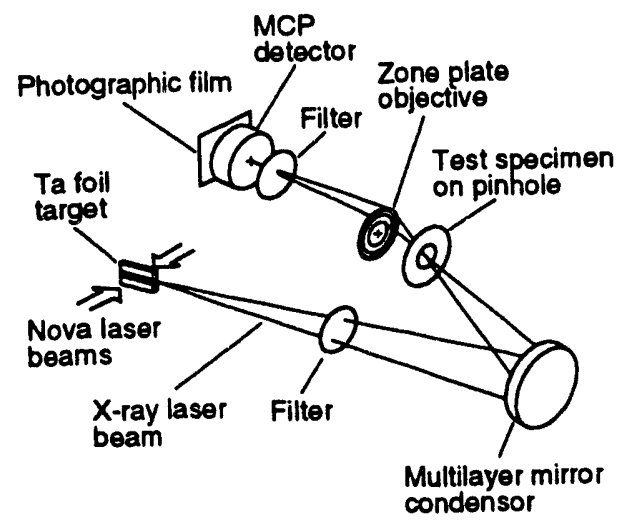

III.E.2-4) Schematic drawing of LLNL x-ray microscope, showing main components.

form a high-temperature plasma with low density gradients. For this experiment, two optical beams from the Nova laser were used to generate a combined intensity on target of $3.0 \times 10^{14} \mathrm{~W} / \mathrm{cm}^{2}$ for a duration of $500 \mathrm{ps}$. The $\mathrm{x}$-ray laser pulse, $200 \mathrm{ps}$ FWHM pulse, is characteristic of exploding foil amplifiers, and the $x$-ray laser originates from a $\sim 100-\mu \mathrm{m}$ diameter gain region at the center of the plasma and has a beam divergence of $10 \mathrm{Mrad}$ FWHM. The output energy is $\sim 10 \mu \mathrm{J}$, which gives a brightness of $10^{21}$ photons $/\left(\mathrm{s}-\mathrm{Mrad}^{2}-\mathrm{mm}^{2}-0.01 \% \mathrm{BW}\right){ }^{19}$ 
Figure III.E.2-5 shows an image of a resolution test pattern consisting of radial gold bars which taper down at the center to $\sim 350 \AA$. The gold bars are 1000- $\AA$ thick and are on a $1000-\AA$ silicon nitride substrate. Features near the diffraction limited resolution of $\sim 550 \AA$ are clearly observed. The nonuniform illumination pattern is due to the finite source size of the $x$-ray laser, which is demagnified onto the test pattern by the multilayer mirror collecting optic. The effective source size is estimated to be $50 \mu \mathrm{m}$ by $80 \mu \mathrm{m}$. The grainy nature of the image is due to the microchannel plate detector and the low signal levels.

Using this $\mathrm{x}$-ray microscope, images have been made of three rat sperm nuclei that have undergone different preparation techniques. The goal was to investigate the advantages of gold labeling and to access the effects of the various preparation techniques.

The nuclei were first prepared by treating sperm isolated from rat epididymides with a disulfide reducing agent and detergent to dissolve the tails, acrosome, and nuclear membranes, exposing the DNA-protamine complex that comprises sperm chromatin.

A droplet containing the amembraneous nuclei was deposited onto a silicon nitride window with dimensions $300 \times 300 \mu \mathrm{m}$ by $1000 \AA$ thick, and the liquid containing unbound nuclei was removed after $30 \mathrm{~s}$.

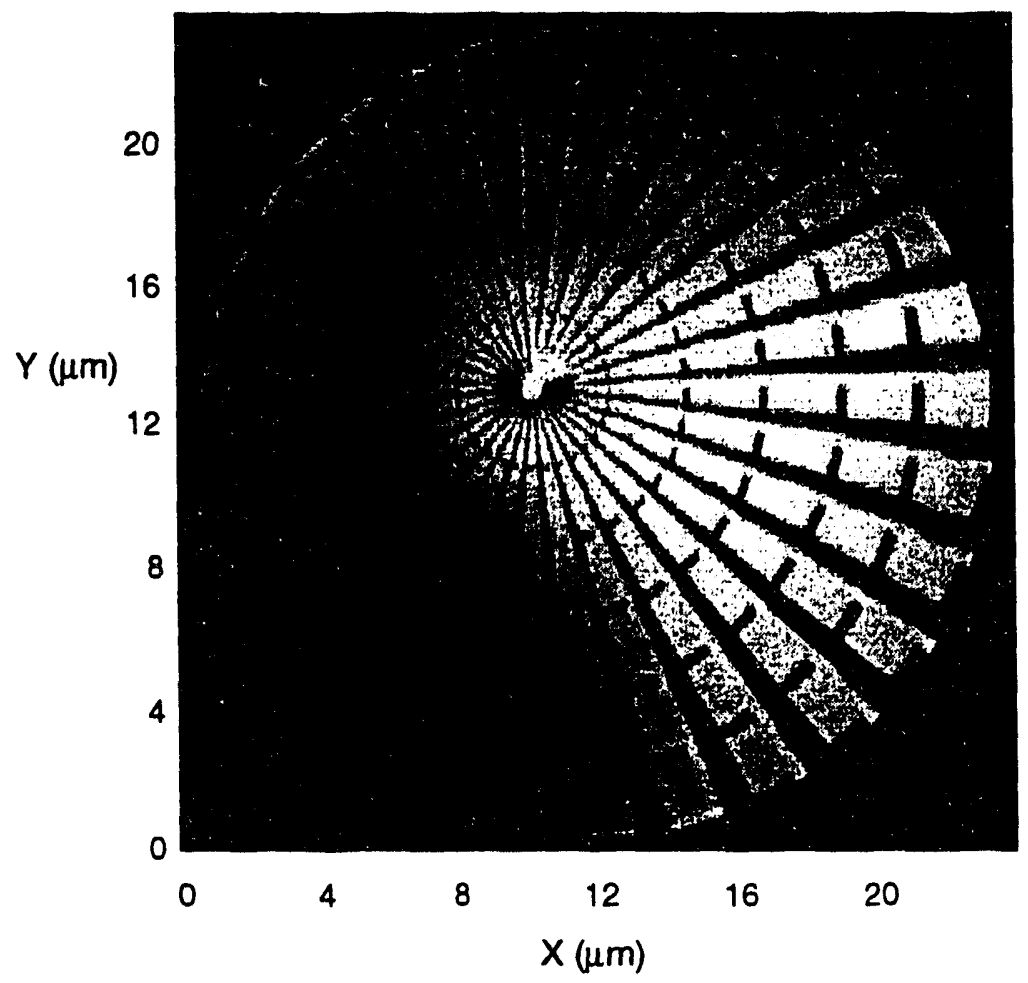

III.E.2-5) Image of resolution test pattern obtained with $x$-ray laser microscope. 
In Fig. III.E.2-6a images are shown of unstained rat sperm nuclei and images "stained" with mouse antiprotamine 1 and antiprotamine 2 antibodies and goat antimouse antibodies tagged with $400-\AA$ diameter gold. The images show distinct differences. The stained images show high concentrations of gold along the edge of the sperm and some evidence of individual $400-\AA$ gold particles on the surface. The clumping of the gold labels is particularly evident in the image of the nuclei stained with the antiprotamine 1 antibody (Fig. III.E.2-6b). The frame structures observed in the antiprotamine 2 stained nuclei (Fig. III.E.2-6b) are a consequence of the preparation technique that unmasked internal sites for antibody binding. These images clearly show the value of gold labeling to enhance contrast, but also show the need for avoiding unnecessary preparation techniques that can lead to artifacts.

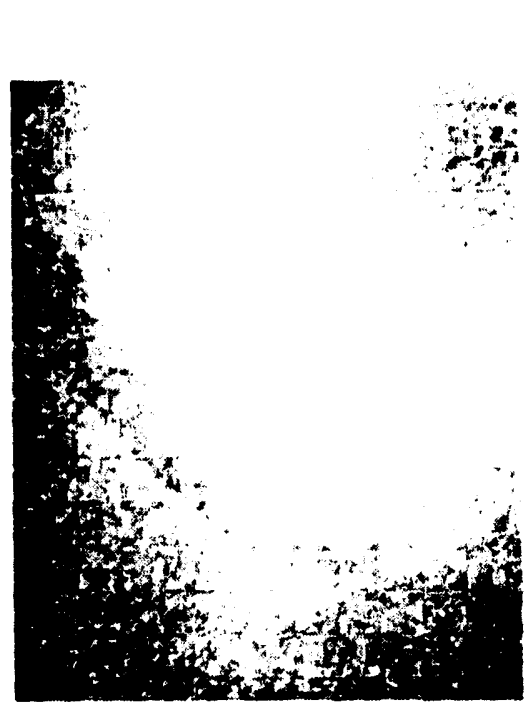

a)

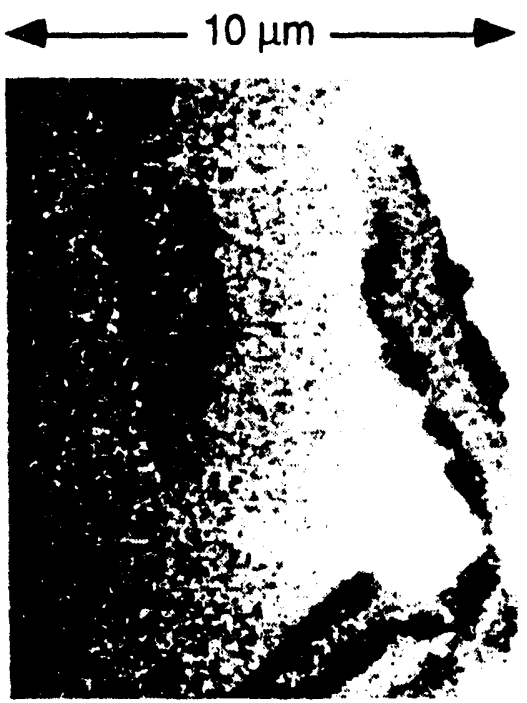

b)

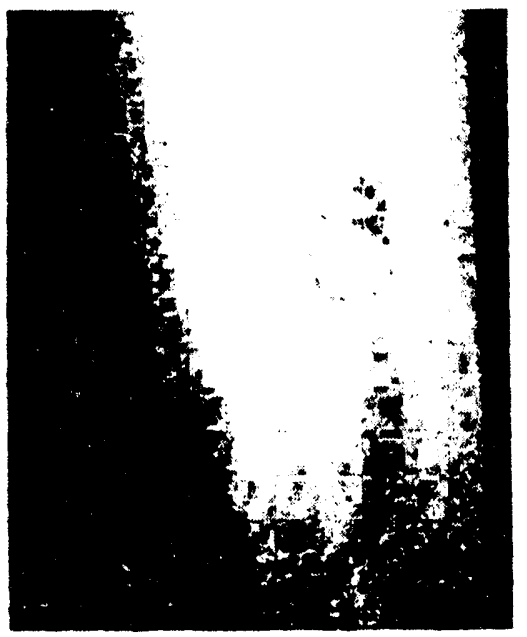

c)

III.E.2-6) X-ray microscope images of rat sperm nuclei a) with no gold labeling, b) stained with antiprotamine 1 and gold labeled, and c) stained with antiprotamine 2 and gold labeled.

\section{F) Material Properties}

\section{1) Equation of State}

Material pressures of millions of atmospheres (megabars or Mbar) to hundreds of millions of atmospheres are common in astrophysical objects, and are observed or predicted in the laboratory. Direct illumination of metals by a Nova-class terawatt laser can produce pressures of $100 \mathrm{Mbar}$ or greater. Gigabar (Gbar) pressures are predicted in spherically compressed capsules typical of inertial confinement fusion targets and 
radiatively driven samples. Because the equation of state (EOS) largely describes how a material reacts to pressure, the thermodynamics and hydrodynamics of these systems cannot be predicted without a knowledge of the EOS for this regime.

Although the EOS of matter in the limiting case of extremely high pressure is expected to be described by a Thomas-Fermi model, the regime of applicability and approach to this limit are not known; EOS data is sparse at pressures above 3-4 Mbar because of the difficulty in producing high-pressure conditions while simultaneously measuring the relevant parameters. The ultimate goal is to use a material of known EOS as an EOS standard material and to obtain EOS data on materials of interest.

In this type of experiment the shock velocity $u_{s}$ and initial density $\rho_{0}$ are measured in each of two materials effectively adjacent to one another. Shock impedance is the product $\left(\rho_{0} u_{s}\right)$. If the EOS of material I is known, then a Hugoniot point (i.e., the locus of final states obtained from passage of shocks of varying strengths through a material) of material II can be derived from the measured shock impedances.

At present there is no material whose EOS is sufficiently accurate to be used as a standard at the pressures of interest. The standards would be qualified by a series of experiments that use paired materials, both of which have equations of state currently under theoretical development in the pressure range of interest. The goal would be to provide accurate experimental data to test theory over a wide range of pressures, densities, and temperatures. The materials to be qualified as standards could be, for example, aluminum and copper, because the theory is under development and because of the relative ease of target fabrication.

To perform this type of primary data experiment in the pressure range of 10-50 Mbar (i.e., 1-5 TPa) requires, for direct illumination, intensities up to $\sim 3 \times 10^{15}$ $\mathrm{W} / \mathrm{cm}^{2}$, while for $\mathrm{x}$-ray drive, a temperature range of about $110-190 \mathrm{eV}$ will be required. To perform much higher pressure experiments would require a substantially larger laser system, for example the NIF. This is a difficult regime, due not only to the accessible pressure regions, but also to the rigid constraints one must observe with respect to the pre-heat, shock velocities measurements, and shock planarity.

There is exploration and development into three techniques that will allow us to obtain EOS data in the Mbar and near-Gbar regimes. All three use high-energy lasers as the driving source to produce intense shocks in materials. Indeed, laser deposition is the only way to presently produce these conditions in the laboratory. As discussed above, the shock brings the material into a high pressure state, albeit a transient one; measurements can then be made on the shocked matter to obtain EOS data points. A primary objective of this work is to produce spatially large, planar shocks that are easy to diagnose and interpret. The shock must be spatially large enough so that the data are unambiguous: very intense shocks can be generated by focusing a laser to a very small spot, but then side motion and potential development of plasma instabilities require multidimensional modeling. 


\section{a) Directly Driven High-Pressure Shocks}

The long history of attempts to produce very strong shocks in materials by direct irradiation with lasers has produced conditions inferred in targets of from 2 Mbar to $100 \mathrm{Mbar}$, but these had problems with two-dimensional effects or extremely uneven laser spatial profiles, which can lead to laser-induced local hot spots and instabilities.

To ameliorate the laser nonuniformities, the laser spot has been smoothed with the use of a random phase plate, a filter with a random pattern of small holes that produces a far field pattern that is even in intensity, within $10 \%$, except on scales smaller than about $10 \mu \mathrm{m}$. In addition, steering wedges have also been used, which steer different parts of the beam in the near field to different spots in the focal plane to produce a more flat-topped distribution.

Employing phase plates and steering wedges on a 1-ns beam of Nova provides an intensity of $3 \times 10^{14} \mathrm{~W} / \mathrm{cm}^{2}$ of $2 \omega_{0}$ light in a focal spot of more than $1 \mathrm{~mm}$ in diameter with a uniformity of $\pm 10 \%$ over the spot diameter. The beam was focused on a $25-\mu \mathrm{m}$ thick aluminum disk with the rear side of the disk (i.e., the side away from the beam) imaged with a UV streak camera. The result was an extremely uniform shock breaking out of the rear of the target.

A schematic of the setup and a streak image is shown in Fig. III.F.1.a-1. Figure III.F.1.a-2 shows that the shock, after traversing the 25- $\mu \mathrm{m}$ thickness of $\mathrm{Al}$ in approximately 650 ps, varies in breakout time across the entire diameter by less than 20 ps, implying that the shock was planar across the disk by to better than $1^{\circ} .20$ Pressures inferred in the shocked aluminum, using stepped targets and wedges, were 20-30 Mbar.

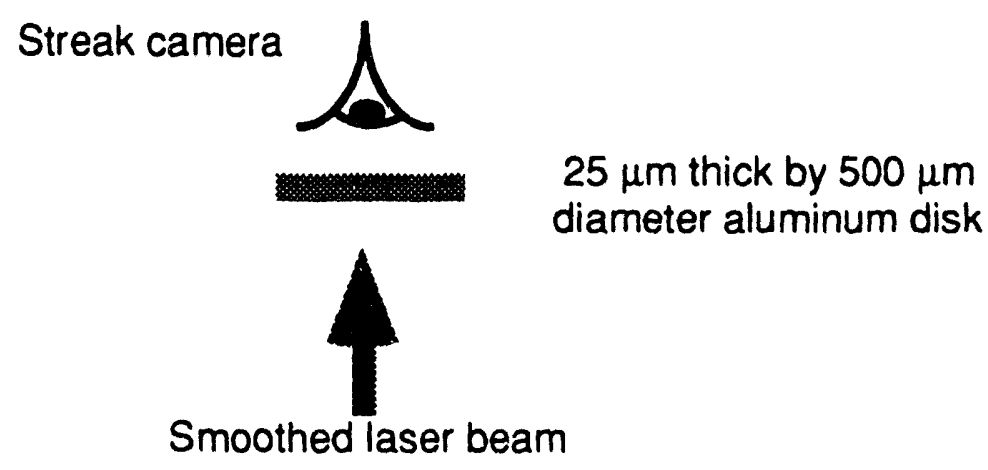

III.F.1.a-1) Direct laser-driven shock experiment. The schematic shows a spatially smoothed beam of Nova irradiating an Al disk, $25 \mu \mathrm{m}$ thick by $500 \mu \mathrm{m}$ in diameter. The schematic also shows the position of the streaked UV imager. 


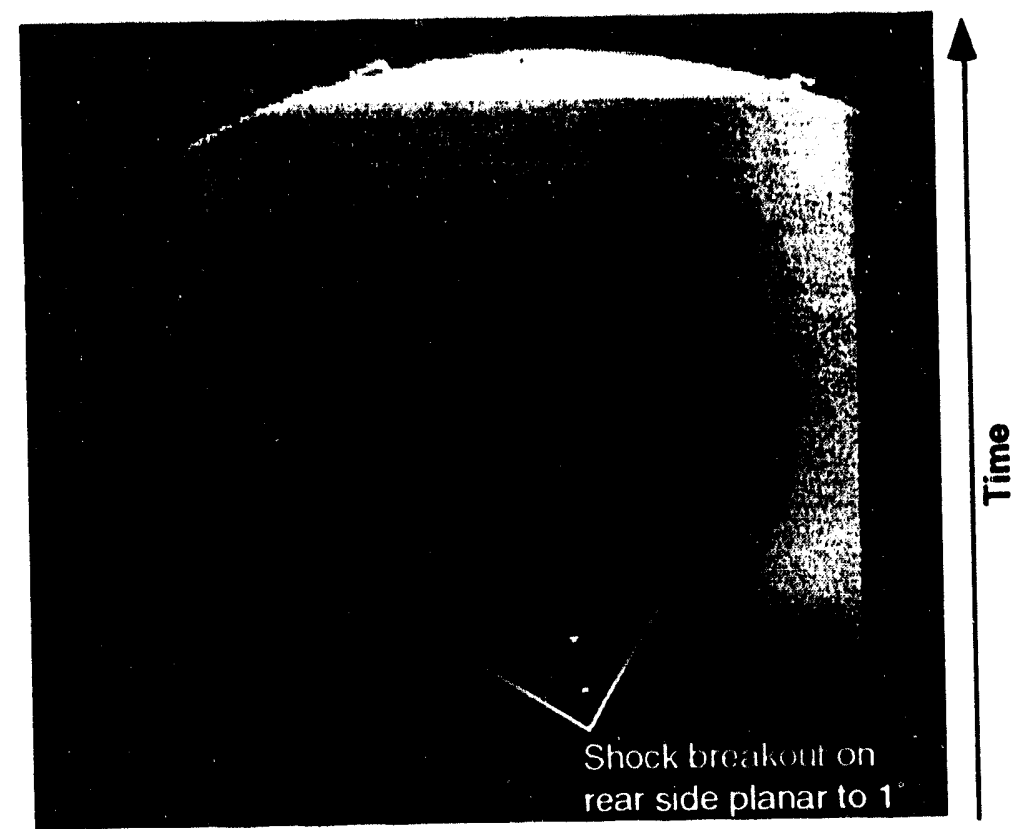

III.F.1.a-2) Direct laser-driven shock experiment image from the diagnostic, showing an extremely planar shock emerging from the rear side of the target.

\section{b) Indirectly Driven Colliding Foil Experiments}

To reach a regime of much higher pressure without sacrificing spot size, and thus one-dimensionality, a very different technique has been employed, a variation and miniaturization of the well-known flyer-plate technique. ${ }^{21}$ In this method, the flyer, that is a foil in the present case, stores kinetic energy from the driver over an acceleration time and delivers it much more rapidly as thermal energy in collision with another foil. In addition, the flyer acts as a preheat shield so that the target remains on a lower adiabat than if it were exposed to the driver. These attributes make it possible to achieve much higher pressures using a flyer-impact configuration than using a directly driven configuration.

In the experiments, an x-ray drive was produced by focusing the ten beams of the Nova laser into a millimeter-scale cylindrical gold hohlraum and utilizing the radiation escaping from a hole in the cylinder. The experimental arrangement is illustrated in Fig. III.F.1.b-1. The hohlraum $x$-rays ablated a $50-\mu \mathrm{m}$ layer of polystyrene to which was attached a 3-mm-thick gold foil. This flyer foil accelerated through a 50-um void region and, near the end of the laser pulse, collided with a stationary gold targt $i$ foil composed of two thicknesses, $2 \mu \mathrm{m}$ and $6 \mu \mathrm{m}$, launching a compression wave into the target foil. The shock on the rear side of the target foil was imaged with an optical streak camera.

The experimental packages were more than $0.5 \mathrm{~mm}$ in diameter and the cylindrical target assembly was mounted across a hole in the wall of the hohlraum with the ablator 

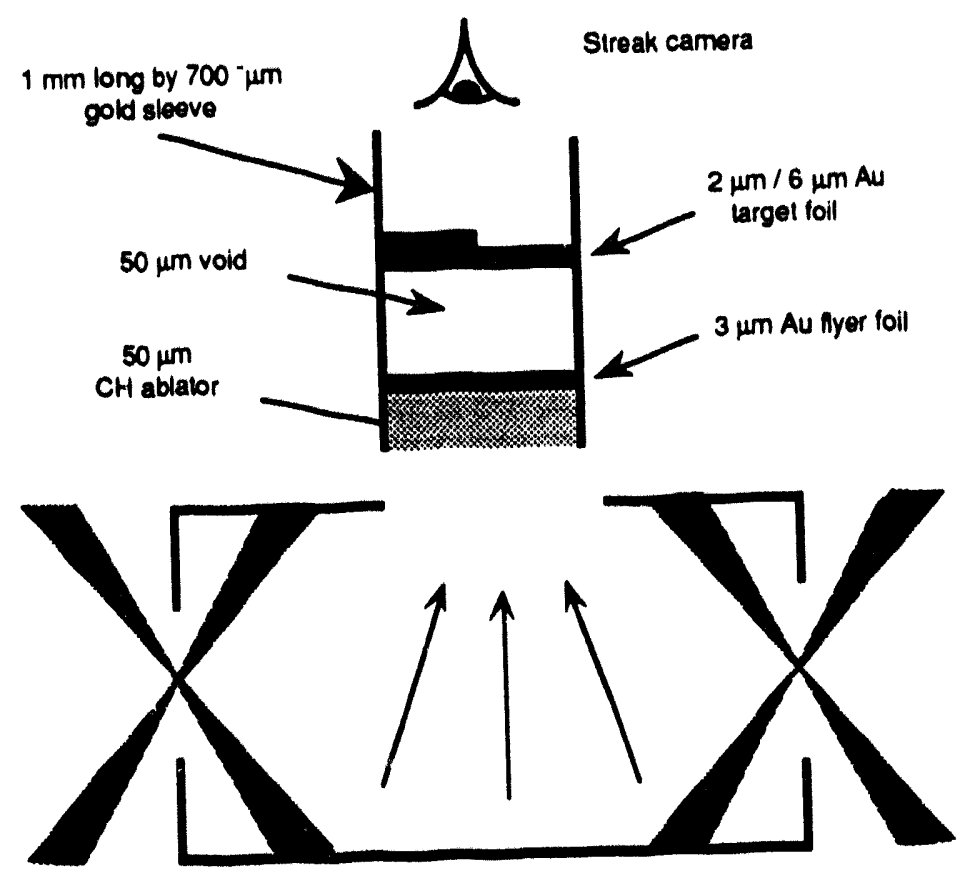

III.F.1.b-1) Radiation-driven shock experiment. The diagram shows the colliding foil arrangement (not to scale). The 1 -mm-long by $700-\mu \mathrm{m}$-diameter experimental package is shown facing the hohlraum $x$-ray drive. Components of the package are a 50-mm CH ablator to which is attached 3-mm Au fyer foil, a $50-\mathrm{mm}$ void, and a two-thickness Au target foil, all held in a gold sleeve.

facing the hohlraum interior. The approximately $100-\mu \mathrm{m}$ long target assembly was placed at the bottom of a 1-mm gold sleeve so that the assembly was completely shielded from unfocused, unconverted laser light. Additional shielding prevented the diagnostic from viewing heated areas of the sleeve and the hohlraum.

A typical streak camera image in Fig. III.F.1.b-2 shows shock breakout at two times corresponding to the two thicknesses; the time interval between the breakout times measures the shock speed in the target if the shock speed is constant. Here, an interval of $57 \pm 5$ ps between breakout on the two thicknesses is measured, corresponding to an average shock velocity of $70 \pm 6 \mathrm{~km} / \mathrm{sec}$. From the SESAME equation-of-state tables, this shock speed corresponds to a density of $90 \mathrm{~g} / \mathrm{cm}^{3}$ and a pressure of $0.74 \mathrm{Gbar}$ in the gold target, by far the highest inferred pressure obtained in the laboratory.

Any slight spatial imbalance in the drive or any unpredicted edge effects, due, for example, to interactions between the flyer foil and the sleeve, could cause the flyer to tilt or curve, which would drive a non-planar shock into the target. However, because of the relatively large diameter of the foils, any non-planarity in shock breakout could be observed. In addition, the step in the target was at the center of the large foil, where the effects of edge-induced nonuniformities would be minimized. 


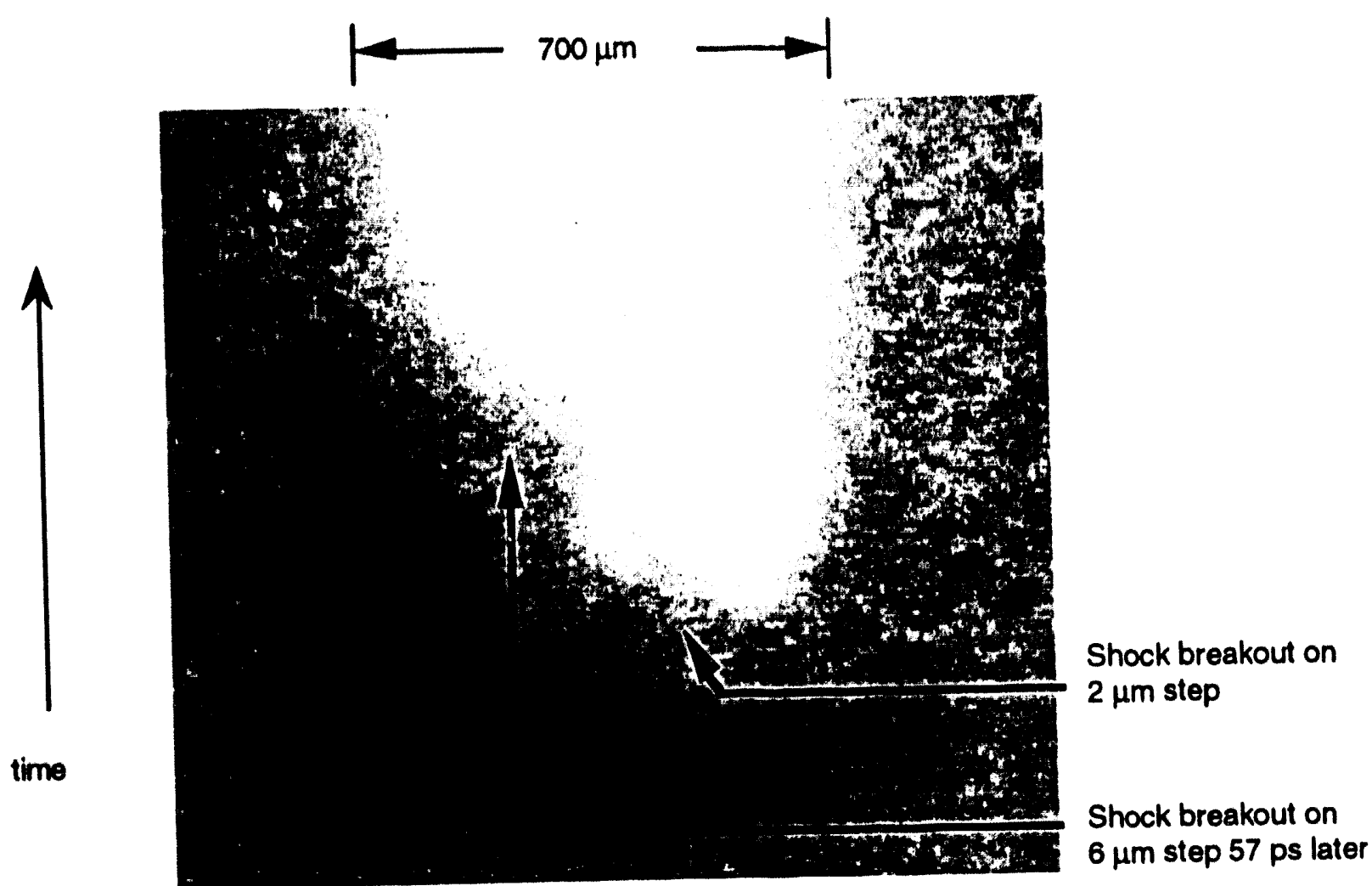

III. .1.b-2) Data image showing shock breakout on the rear surface of a two-step target foil at two distinct times corresponding to the two thicknesses of the target. This time interval provides the shock travel time across the $4-\mu \mathrm{m}$ step difference. The interval is about $57 \mathrm{ps}$, indicating a shock speed of $70 \mathrm{~km} / \mathrm{sec}$.

If the target foil were preheated by high-energy $x$-rays from the hohlraum before the flyer-target collision, the measurement would be compromised. To test this possibility, the $x$-ray drive was altered in one experiment so that the overall drive intensity was identical to the that in the other experiments, but the intensity of high-energy $x$-rays, those $\geq 2.5 \mathrm{keV}$, was reduced by more than a factor of five. The result was well within the uncertainties of the other experiments, so the results are not affected by preheat.

A straightforward extension of this technique can be made to obtain EOS data for this regime. If the flyer foil can be shielded so that the flyer does not significantly heat or decompress, EOS data points can be found by measuring the speed of the flyer foil. Since it has been demonstrated that the target foils do stay intact, this can be accomplished by modifying the target foil as shown in Fig. III.F.1.b-3.

In addition to the shock speed measured as above, the flyer speed can be simultaneously obtained by recording shock breakouts from two identical foils placed at different distances along the flyer path. One experiment was performed with a three-step $\mathrm{Au}$ target foil. Although the condition of the flyer foil was unknown, so that an EOS data point could not be evaluated, observed shock breakout from the three steps indicates the viability of the approach. To proceed with EOS measurements, the condition of the 


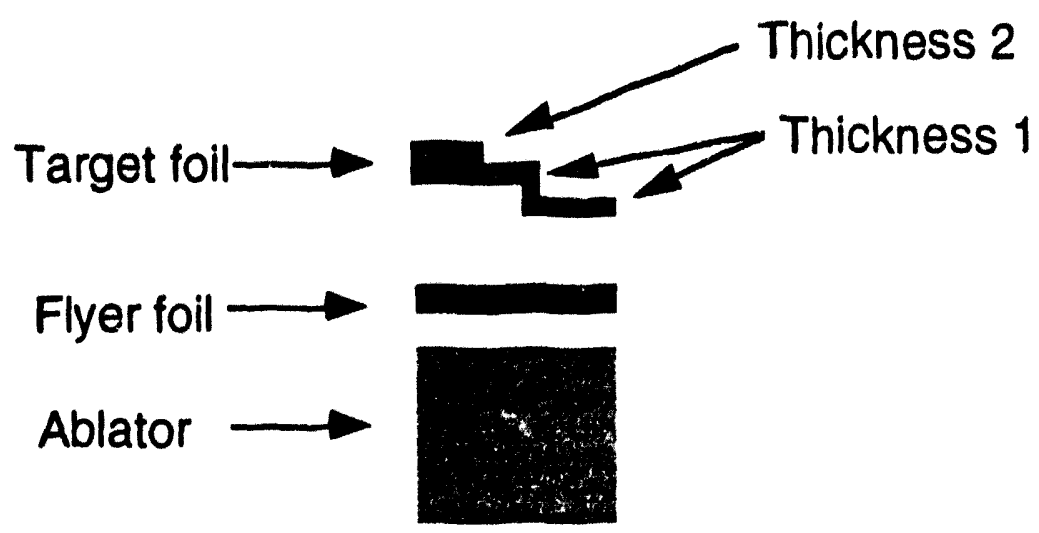

III.F.1.b-3) A target foil configuration that will allow the simultaneous measurement of shock speed and flyer speed. From the latter, the material speed behind the shock can be inferred. These two measurements will allow the calculation of an EOS primary data point in the Gbar regime.

flyer foil must be known; indeed the accuracy of the result may depend on the flyer remaining solid. The development of a high-magnification $x$-ray laser radiograph technique to determine the conditions of accelerated foils has been undertaken and this is described in the section on X-ray Laser Applications.

\section{c) Indirectly Driven Shock Experiments on Polystyrene}

Plastics are very different materials than metals, but they are just as pervasive in high-energy laser experiments as major constituents of diverse targets. Since plastics, unlike metals, are largely transparent to high-energy $x$-rays, $x$-rays can be used to backlight relatively thick samples of $\mathrm{CH}$ and provide information on the sample as a function of time. In particular, the shock front in a sample of $\mathrm{CH}$ can be seen on a streak camera by simultaneously imaging the transmission of an $\mathrm{x}$-ray backlighter through the shocked and unshocked material; the transmission through the denser, shocked $\mathrm{CH}$ is significantly less than through normal density $\mathrm{CH}$, that is, the transmission is about $70 \%$ less for a compression of 4 in a $0.7-\mathrm{mm}$ thick sample using a $7-\mathrm{keV}$ backlighter energy. If, at the same time, motion of the material behind the shock can also be imaged, a data point in the EOS is obtained.

An experimental arrangement, adapted from an experiment to measure material mix at interfaces ${ }^{22}$ which accomplishes this, is depicted in Fig. III.F.1.c-1. The material motion behind the shock is viewed as the difference in transmission of the backlighter through shocked, doped $\mathrm{CH}$ and shocked, undoped $\mathrm{CH}$; this is the interface shown in the figure. The drive, as $\mathbf{x}$-rays, is provided by a hole in the side of a gold hohlraum.

Here only eight beams of Nova are used for the drive, saving two beams for the backlighter. Code simulations predict a pressure of $\leq 40 \mathrm{Mbar}$ near the interface. Two experiments have been performed, both with $2 \% \mathrm{Br}$ doped into the first section, which 


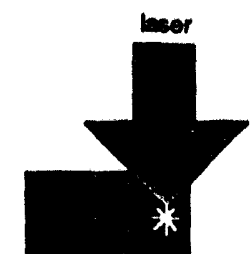

Area backlighter

\section{Shock front}

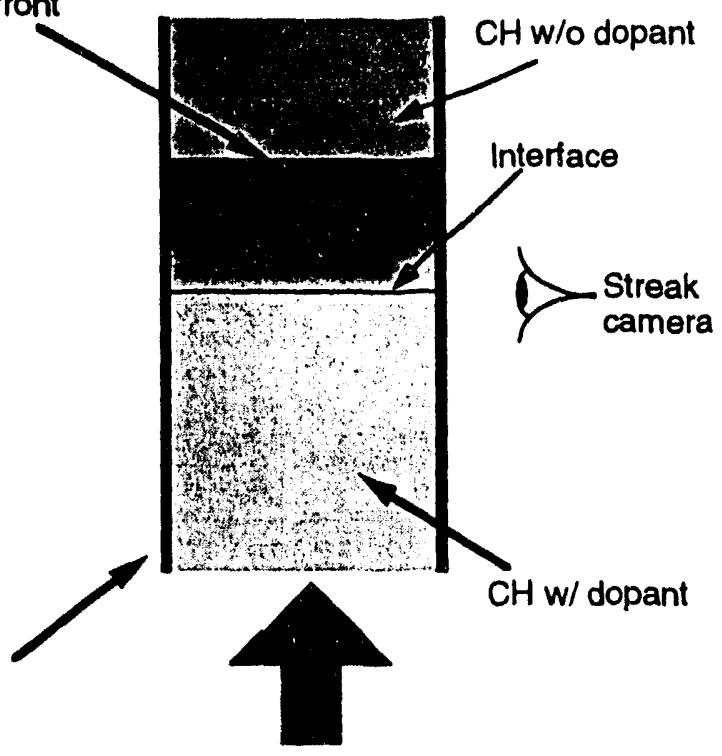

Hohlraum $x$-ray drive
III.F.1.c-1) Overview of the arrangement for radiationdriven shock experiments in transmissive media, showing relative positions of the package, mounted across a hohlraum, backlighter, and streak camera. Differences in transmission of the backlighter through the package will allow measurement of both the shock speed and the speed of the interface between doped and undoped plastic. This will be a direct measurement of the EOS of $\mathrm{CH}$ in the multi-Mbar regime.

was $300 \mu \mathrm{m}$ long. A 3-ns long backlight beam was initiated at about the time the shock was expected to pass through the interface. Although the interface was clearly visible on the streak records, the shock front was too weak to be seen. However, this technique will allow EOS data to be obtained on $\mathrm{CH}$ in the multi-Mbar regime.

\section{2) Opacity}

Knowledge of the $x$-ray opacities of hot matter is essential for the understanding of the plasma state and for radiative transport. The Nova laser has been used to obtain experimental, high-quality opacity measurements. This required the development not only of high-resolution spectroscopic techniques to measure the x-ray transmission of the opacity samples, but also required the development of techniques to accurately and simultaneously measure the temperature and density of the samples. Simply, to provide a benchmark of the ability to measure an LTE opacity, it is required that one perform measurements on relatively simple atomic species first, thus with some degree of faith in the opacity predictions the technique can be evaluated. This required exacting procedures that provide the temperature, density, and gradient scale lengths on a single experiment.

The x-ray absorption of the opacity samples was obtained by the method of point projection spectroscopy, which is illustrated in Fig. III.F.2-1. In this technique, eight of the laser beams at Nova are used to heat the opacity sample. Then, a point source of $x$-rays is produced by tightly focusing one of the remaining laser beams onto a small backlight target of high- $Z$ material. $X$-rays from the backlight pass through the opacity sample onto an $x$-ray diffraction crystal and are then recorded on $x$-ray film. Other 


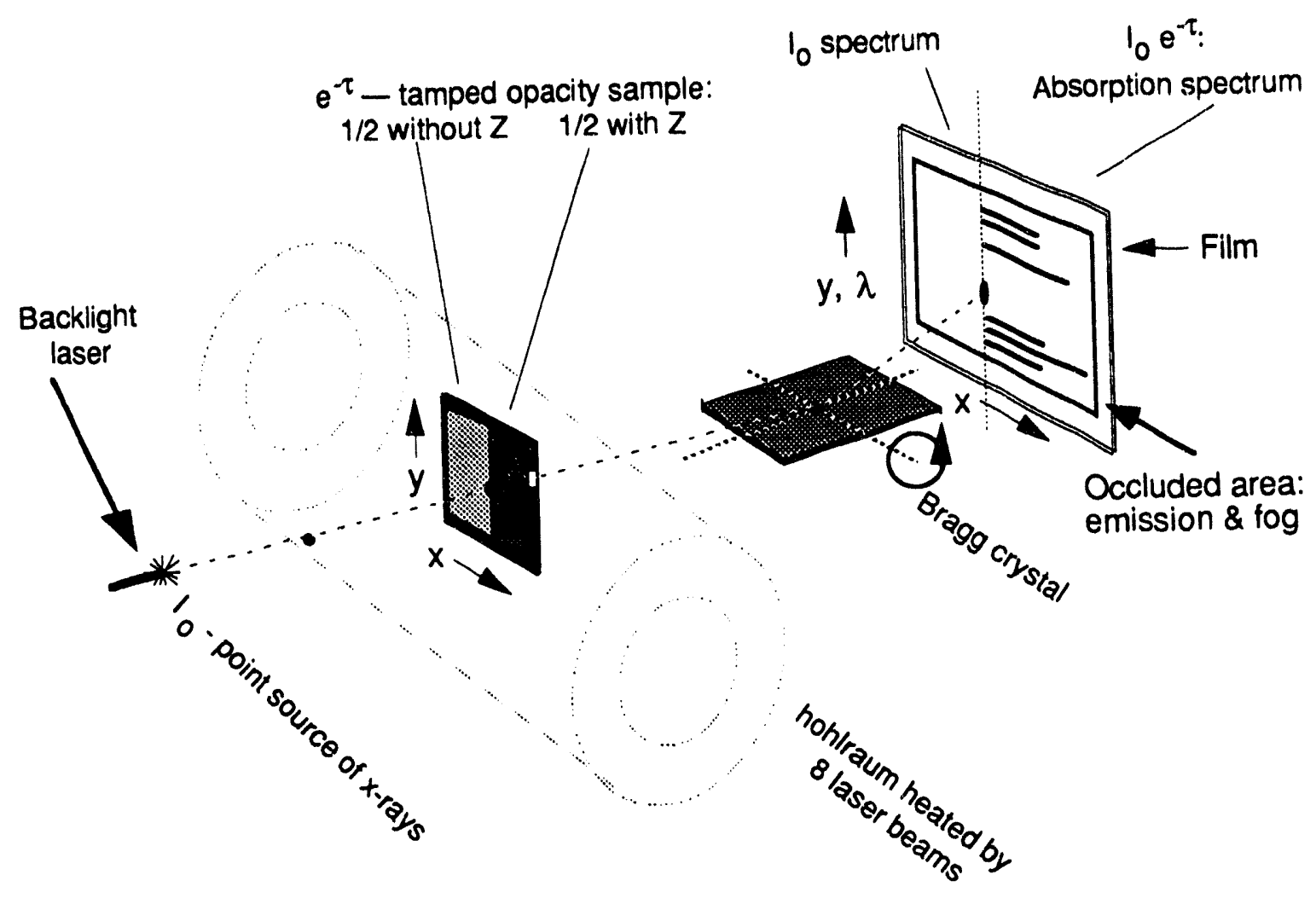

III.F.2-1) Schematic of the point projection spectroscopy for opacity measurements. The laser-produced backlight $x$-rays pass through the target and are imaged. Since the crystal disperses the spectrum, one obtains a spatially and spectrally resolved image. Temporal resolution is provided by backlight duration.

$x$-rays from the same point backlight bypass the sample, but are still diffracted from the crystal onto the film record. The ratio of the $x$-ray spectrum attenuated by the sample to the unattenuated $x$-ray spectrum provides the $x$-ray transmission spectrum of the sample. Proper collimation allows a highly quantitative analysis of the spectrum. Backgrounds from film chemical fog, sample emission, and crystal x-ray fluorescence can all be separately determined from the $x$-ray film record.

Careful attention was paid to the sample conditions. It was required that the sample be uniform throughout in both temperature and density. Uniformity of temperature was attained by heating the sample in a special hohlraum. The hohlraum was constructed so that no laser light impinged on the sample directly, or on first reflection; thus, the sample was heated only by $x$-rays. The hohlraum also kept a uniform temperature throughout the sample, and along with the relatively high density of the sample ensured that the sample was in local thermodynamic equilibrium, LTE.

Further, the samples were tamped by plastic so that as the sample expanded the density throughout the sample was constant. The thickness of the tamper was determined by hydrodynamic calculations and density uniformity was checked in the experiments. This experimental technique allowed the measurement of the Al opacity and laid the basis for using the $\mathrm{Al}$ opacity as an independent temperature diagnostic. 
In further experiments, the density of the samples was determined by imaging the samples. This was done by using a second point-projection spectrometer to image the expansion of the samples. This spectroscopic record also was used to determine that the samples had uniform density throughout the sample. The temperature of the sample was determined by mixing the sample with aluminum. A third point-projection spectrometer was used to measure the absorption of the aluminum $n=1$ to $n=2$ transitions. The relative intensities of the transitions from the different ion species gave the ion balance in the aluminum which (coupled to the density measurement) gave the temperature of the sample.

These techniques allowed the density to be measured to an accuracy of $\pm 20 \%$ and the temperature was measured to an accuracy of $\pm 5 \%$. With these accuracies it was possible to make quantitative comparison between the experimental results and the theoretical calculations of the opacity. ${ }^{23}$

In particular, one experiment measured the opacity of niobium. The niobium sample contained $14 \%$ Al by weight for the temperature measurement described above. Figure III.F.2-2 shows in a) the transmission of the aluminum and in b) the transmission of the niobium. The dotted lines overlaying the experimental data are the calculations. In general there is excellent agreement.
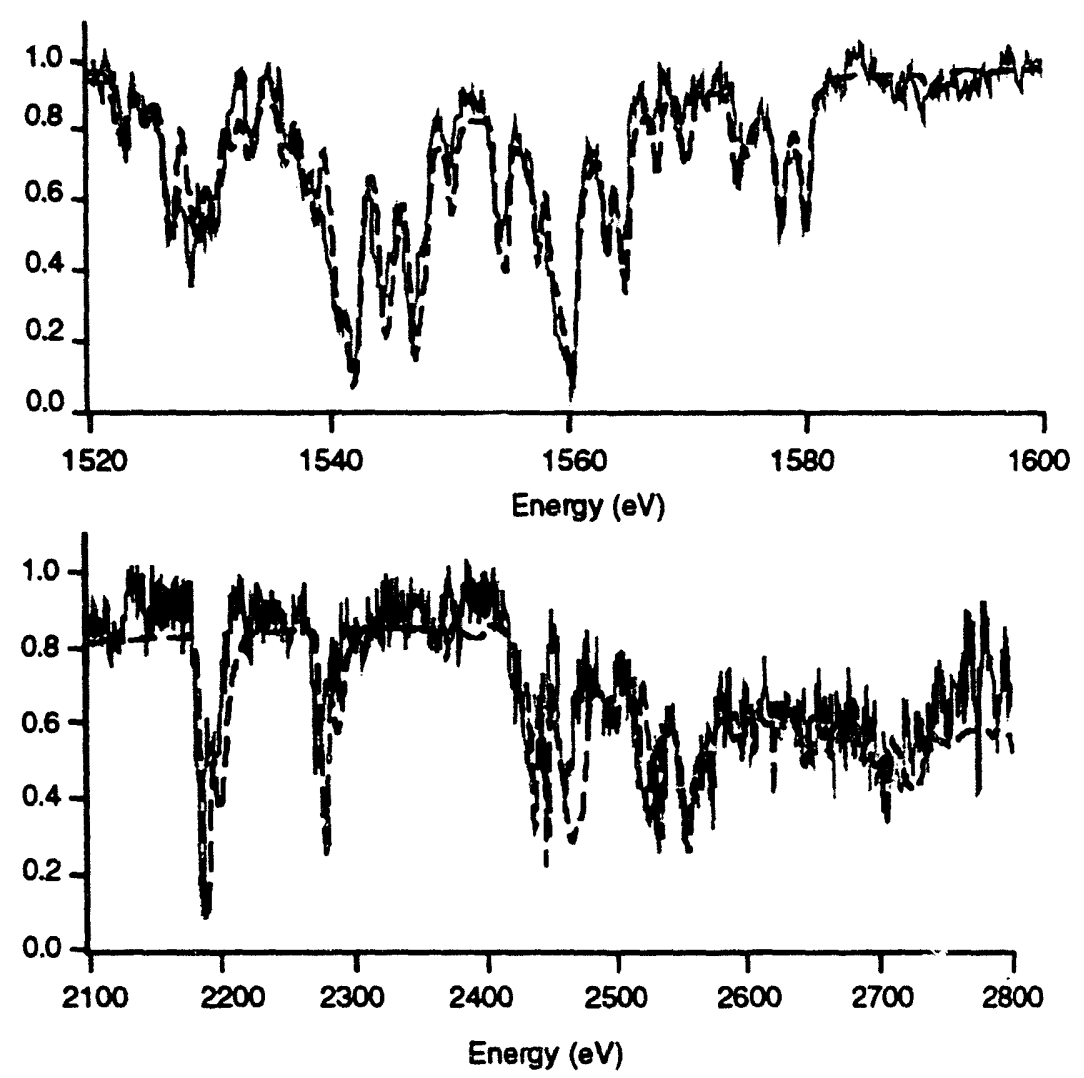

III. 2-2) Absorption of the AlNb sample. The experimental data are in the solid gray line and the OPAL LTE opacity prediction is the dashed line. a) The spectrum of the AI K $\alpha$ lines which have been previously verified to yield an accurate temperature measured on the same experiment as b) the $\mathrm{Nb}$ spectrum. 
This experimental result defines a milestone in that we have proven the ability to measure the opacity of the aluminum sample with sufficient accuracy that it can now serve as an in situ temperature diagnostic for the sample. The accuracy of the temperature, measured to be $48 \mathrm{eV}( \pm 2 \mathrm{eV})$, indicates an important advance on previous attempts to measure temperatures of high-energy-density matter.

In other experiments on opacity the astrophysically important $F e D n=0, n=3$ absorption feature, that causes a large increase in the opacity above older predictions, nas been confirmed. ${ }^{24}$ The work on iron has been further extended to measurement of the spectrum in sufficient detail to evaluate the Rosseland mean for comparison with theoretical predictions. ${ }^{25}$ Both of these studies on Fe opacity are motivated by astrophysical considerations and indicate that, in at least this one area, the experimental capability has become mature.

\section{3) Strength of Material}

Laser generation of multikilobar shocks has several applications to the study of high explosives, high-velocity impacts, and the alteration of the mechanical properties of certain alloys and ceramics. There have been several important advances in the field since the early 1970 s. Some of the most common methods of studying such shock waves include interferometric methods or the use of piezoelectric transducer gauges. However, these methods yield little information on the dynamics of the near-front face, where the shock wave is initiated.

It is possible to measure the shock strength, in terms of compression ratio, and the spatial density profile using an $\mathrm{x}$-ray diffraction technique for laser-induced shocks for pressures from $1 \mathrm{kbar}$ up to approximately $0.5 \mathrm{Mbar}$, where shock melting occurs, within various crystalline materials. The technique is ideally suited to observation of the dynamics of the front surface of laser-shocked materials. The technique can make a significant contribution to our understanding of shock launching, and such a direct measurement of the relevant parameters of the near-front surface can enable conclusive investigations of the response of novel materials. Extension of the technique to probing the rear-surface breakout of the shocked material is also possible.

When a crystal is subject to strain, its $\mathbf{x}$-ray diffraction properties are altered; most notably the $x$-ray diffraction peak of the crystal is broadened. The physical reasons for this are simply understood: a strained crystal essentially has a distribution of interatomic spacings at which the Bragg condition can be satisfied. The shape of the diffraction peak is therefore a function of the spatial strain profile, and this fact has been used to study strain profiles in crystals caused by dopants, and by laser annealing. The transient alteration of the diffraction peak has also been suggested as a means of obtaining subnanosecond $\mathrm{x}$-ray switching.

The experiment proceeds as follows (see Fig. II.F.3-1). A shock is launched into the crystal of interest. At some point during the shock launching a short (100-ps) pulse of $\mathbf{x}$-ray line radiation produced by a synchronous, but delayed, laser beam irradiating a 

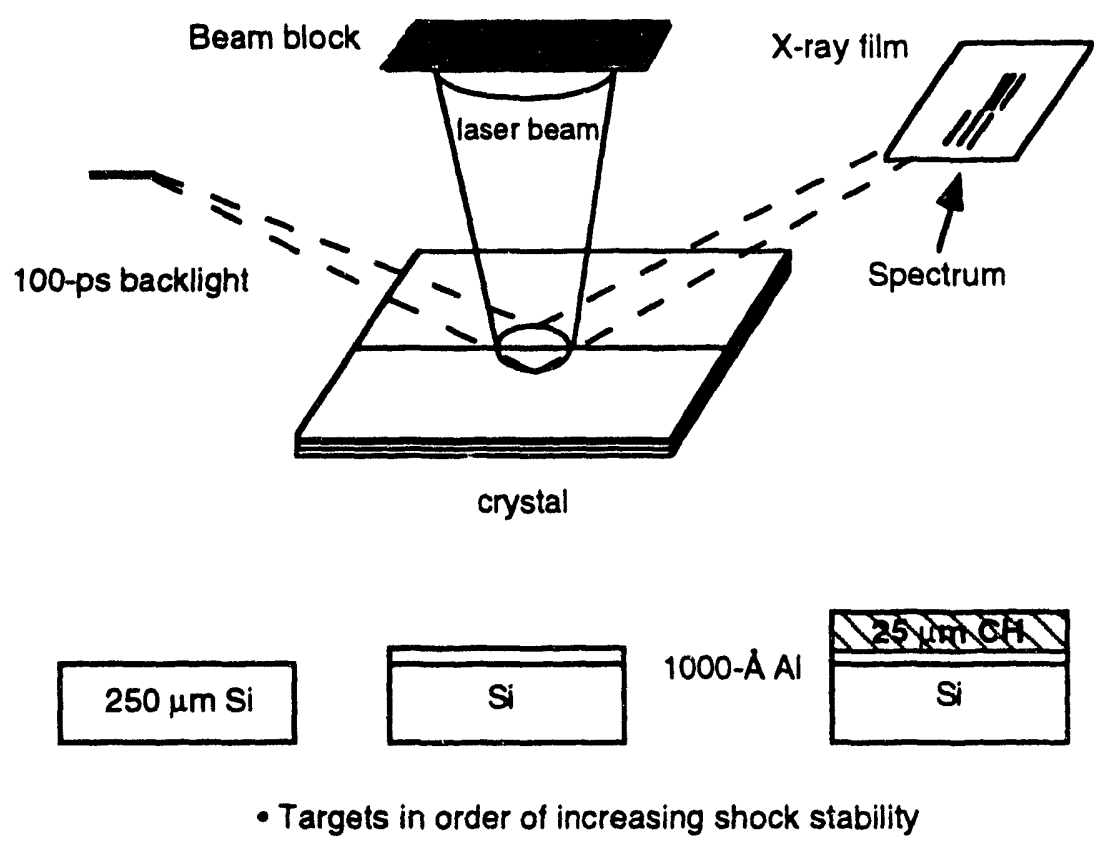

III.F.3-1) A schematic of the compression of a crystal using a laser-induced shock, and the probing of the shock structure using a monochromatic $x$-ray source. Since part of the crystal is shocked and part unperturbed there is a reference spectrum on the film record.

separate target, is Bragg diffracted off the front surface of the shock compressed crystal. The compression of the crystal changes the Bragg condition according to the formula

$$
\Delta \mathrm{d} / \mathrm{d}=-\operatorname{Cot} \theta \Delta \theta
$$

Thus, the line radiation is diffracted to a higher angle than that for the unperturbed crystal, and this angular shift directly indicates the change in the interatomic spacing within the shocked region. As there will be a density gradient at the crystal surface, there will be a range of angles at which diffraction takes place, in addition to the overall shift. Thus, information about the density gradient as well as peak density can be obtained. Indeed it is possible to extract a density profile from the data contained in the diffracted pulse.

The primary interest in this technique would be to find a method of studying the materials under extreme conditions. The present set of known experiments is restricted to simple structures with small heated areas. To develop larger perturbed areas and study the materials at greater depth would require a substantial effort in the development of larger lasers and $x$-ray sources for probing.

\section{a) simple compression studies}

First, silicon has been shocked and transient strains of up to $10 \%$ have been observed. The method of Bragg diffraction relies on the fact that the crystal is remaining "single," i.e., all the planes running parallel to the shock front are compressed 
uniaxially, and the periodicity of the crystal as a whole is maintained. ${ }^{26}$ In some ways it is quite surprising that such an ordered state exists so far above the Hugoniot Elastic Limit, which for silicon in the (111) direction corresponds to an elastic compression of about $2.6 \%$. Thus, one simple experiment is the investigation of the maximum observable compression for a series of different crystalline materials, and comparison of this with shock melting data obtained by more conventional means.

It is interesting to note the utility of high-energy lasers for the following two reasons. First, they provide for a relatively high shock strength within the material of interest, 1 Mbar. Although smaller lasers could attain local pressure of this magnitude, it could only do so over a spot size on the order of a square millimeter. A large spot is needed so that the shocked region of the crystal subtends a large range in angles to the $x$-ray source. Second, small lasers may not have sufficient backlight energy to produce hard enough $x$-rays. It has been found that at large compressions, such as the $10 \%$ compressions in silicon, the $\Delta \Theta$ becomes large for softer $x$-rays and the $x$-rays diffracted from the shocked portion of the crystal get smeared out on the $x$-ray filming, making diagnosis difficult. The change in Bragg angle for harder $x$-rays is not so great, facilitating diagnosis. For example, for the $10 \%$ compressions in silicon, the He-like Ti resonance line was an appropriate $x$-ray source. Much harder x-rays may be required to measure compressions close to shock melting (30-40\%) and for investigating crystals with shorter interatomic spacings, such as lithium fluoride and quartz.

\section{b) Plasticity and Adiabatic Shear Bands}

What the behavior is of a single crystal on the lattice level when it is shocked above the Hugoniot Elastic Limit and, for example, how long it takes to start to deform plastically, are questions that should also be experimentally ripe. 27

There is evidence that some materials, when shocked above the Hugoniot Elastic Limit, form small bands of micron or sub-micron dimension where the dislocation density is extremely high; such areas are known as adiabatic shear bands. Evidence for such localized yielding is mainly based on recovery experiments rather than in situ observation. It is of interest to attempt to observe these bands by short-pulse $x$-ray topography experiment, using a point source of laser-plasma-produced $x$-rays. Point sources of $5 \mu \mathrm{m}$ dimensions have routinely been produced using the laser and could be used to image shear bands. Although the source size is larger than the width of the bands, the bands can run several microns into the crystal, and imaging should be possible in the same way that dislocations of Angstrom size can be imaged using conventional $x$-ray topography.

It is also of interest to determine the shape of the unit cell as the crystal is compressed above the Hugoniot Elastic Limit. This can be performed by using simultaneous Bragg and Laue diffraction with thin crystals. Bragg diffraction takes place from the surface of the crystal, the surface being parallel to the shock front, and measures the elastic compression. At the same time observing the Laue diffraction through the crystal, interrogating the planes running perpendicular to the shock front, provides information about the plastic component of strain. 
Experiments have been performed using $50-\mu \mathrm{m}$-thick silicon (111) crystals, with Laue diffraction off a (220) plane through the crystal. Although both Bragg and Laue lines were observed when no shock was present, the intensity of the Laue line was not sufficient to enable diagnosis when the crystal was compressed. Improvements in crystal preparation and an increase in backlight energy should permit measurements of this type.

\section{c) Metastable Phases}

It is believed that many crystals, when shocked above the phase change point, proceed to a metastable phase before completion. One example is $\mathrm{KCl}$, which undergoes a phase change from $\mathrm{NaCl}$ structure to $\mathrm{CsCl}$ structure at a pressure of about $20 \mathrm{Kbar}$. Investigations of the multiple wave structure in $\mathrm{KCl}$ have been interpreted as transformation to a metastable phase within $2 \mathrm{~ns}$, with completion to the $\mathrm{CsCl}$ structure taking between 25 and 500 ns depending on crystal orientation.

Investigations of $\mathrm{KCl}$ have been performed, and it was found that the diffraction lines from the $\mathrm{NaCl}$ phase disappeared upon shocking. However, the exact strain at which the disappearance occurred was not determined. Time-resolved $\mathrm{x}$-ray diffraction from the shocked crystal could permit measurement of the strain at which diffraction disappeared, which is expected to be at the phase change point. Thus, one would simply "streak" the diffraction.

Disappearance of the diffraction record suggests that the new phase is polycrystalline, as one would expect in an order-disorder transition. Thus, the ability to obtain diffraction information from powders would be of importance.

\section{d) Phase Change Measurements}

The first, and only, in situ x-ray diffraction evidence for a shock-induced phase transition is that obtained from shocked boron nitride, which transforms to a wurtzite structure. It is a displacive transition without a change in unit cell volume or occupancy. Such transitions should take place on an inverse phonon time, i.e., a picosecond time scale. Obviously it would be interesting to repeat and extend these measurements with the improved time resolution and synchronization available with high power laser technology.

In addition to shocking $\mathrm{BN}$, it is interesting to investigate $\mathrm{TeO}_{2}$. This material undergoes a transition from tetragonal to orthorhombic at a pressure of $9 \mathrm{Kbar}$. This is interesting because the transition proceeds by an acoustic shear wave phonon traveling along (110) at an unusually slow sound speed of about $1 \mu \mathrm{m}$ per ns.

\section{e) Rear Surface Studies}

Dynamic tension can be produced within the body of a material by subjecting the front surface of a planar target to impulsive loading. Tensile strain is then induced when the duration of the pressure pulse, $\tau$, is less than $c d$, where $c$ is the shock velocity 
within the material, and $d$ its thickness. In this case two rarefaction waves cross; one is produced at the front surface as the pressure loading falls off, and the other is produced at the rear surface as the incident compression wave is reflected. For sufficiently high tensile stresses a section of the rear portion of the target separates, or "spalls," from the bulk.

At laser irradiances $>10^{9} \mathrm{~W} / \mathrm{cm}^{2}$, absorption of the laser light produces a highpressure plasma at the target surface. As this plasma expands away from the surface, momentum balance launches a compression wave into the bulk of the target. The temporal profile of the compression wave is similar to that of the laser pulse. Direct measurements of tensile elastic strain in silicon (111) wafers have been obtained at strain rates above $108 \mathrm{~s}^{-1}$. Tensile elastic strains of $3.4 \%$ have been directly observed by in situ picosecond $x$-ray diffraction. ${ }^{28}$ This may constitute a novel method of addressing the ideal strengths of materials. A schematic of the rear surface probe experiments is shown in Fig. III.F.3-2.

The experimental results are shown in Fig. III.F.3-3, where the x-ray streak camera record shows the entire history of the rear surface from unshocked, to the initiation of the breakout, through to the time when the crystal is in pure tension. From Fig. III.F.3-3 it can be seen that at early times, for the first $\sim 200$ ps, the $x$-rays are probing the crystal

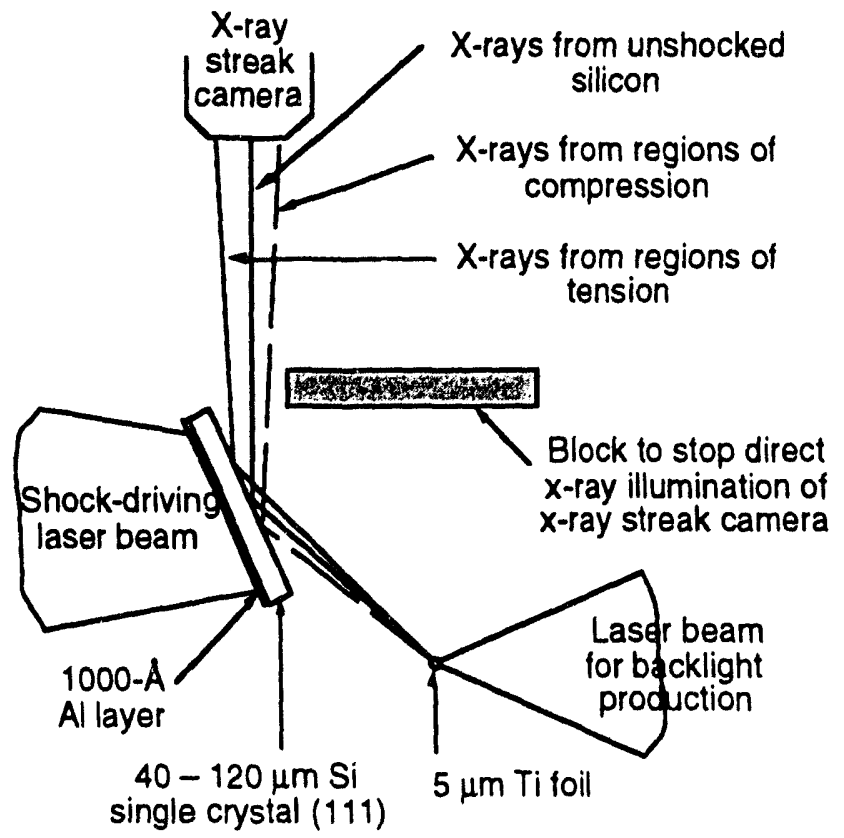

III.F.3-2) A schematic of the rear surface shock breakout experiment-the probing of the shock structure using a monochromatic $x$-ray source. Prior to the breakout, the probed rear surface will be in compression,during the breakout there will also be tension due to a rarefraction wave, and finally the rear surface will go into tension with no compression. 


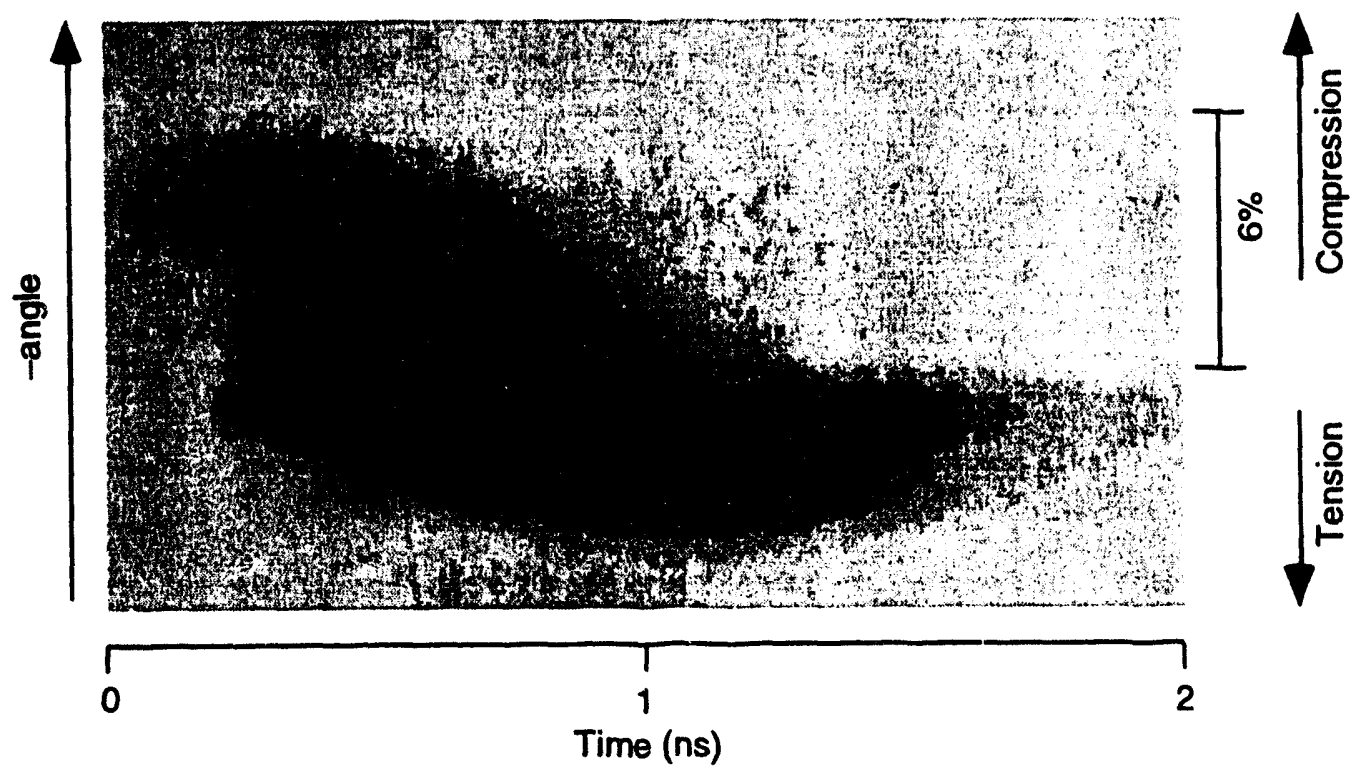

III.F.3-3) Data from the rear surface of a material that has a shock transiting. The streak camera record shows the compression phase at early time as the shock nears the rear surface, and both the compression and tension after the shock breaks out.

in a state of compression, the angular shift in diffraction corresponding to a maximum compression of $6.2 \pm 0.2 \%$. Later the $x$-rays are being diffracted from both regions of compression and regions of tension, and finally, at late times the $x$-rays are being diffracted from a region of pure tension, with a maximum observed tension of $3.4 \pm 0.2 \%$.

These three regimes correspond to the times when the reflected wave has not yet collided with the rarefaction wave due to release of pressure at the front surface; the time during collision of the rarefaction waves; and the time when the reflected wave has passed the front surface rarefaction wave by at least the probe depth of the $x$-rays.

The spectra from a shocked crystal are compared with those from an unshocked crystal in Fig. III.F.3-4. Thus, within the target the strain has changed from $6.2 \% \mathrm{com}$ pression to $3.4 \%$ tension within $\sim 600 \mathrm{ps}$, i.e., at a strain rate of $1.6 \times 10^{8} \mathrm{~s}^{-1}$. The maximum observed tensile strain $3.4 \%$ corresponds to a uniaxial tensile stress of approximately $70 \mathrm{kbar}$, comparable to the largest fracture stresses observed in static measurements.

Finally, it is of interest to point out that with the use of a powder diffraction camera and a bright source (very bright indeed), one should be able to extend all these studies to non-crystalline materials. This would open the way for a new source of studies of the details responses of a much wider range of materials. 


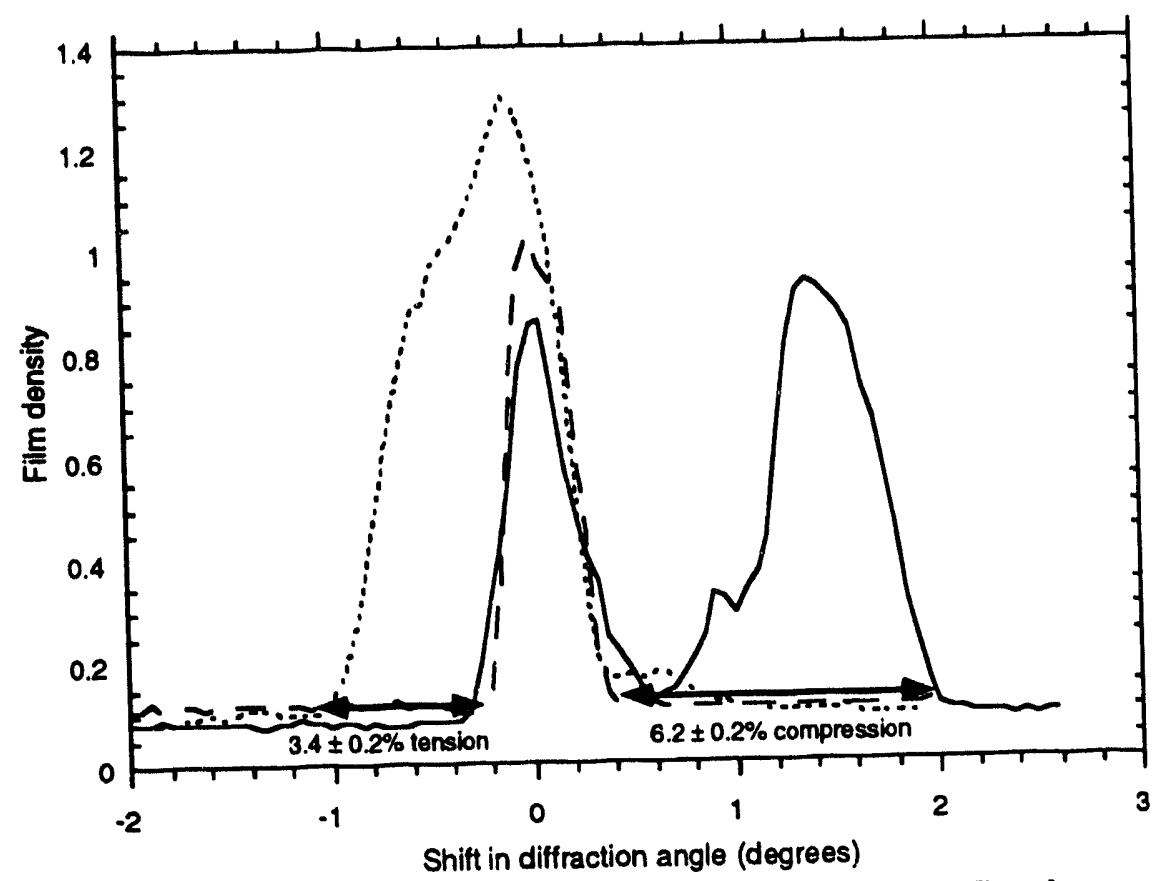

III.F.3-4) Traces showing the spectra from the probed shocked rear surface. The three generic periods are shown - the pure unperturbed crystal in a dashed line; the period of shock breakout, when there is tension and compression, in a solid line; and the pure tension late time phase in the dotted line.

\section{G) Hydrodynamics}

The interest in performing hydrodynamic experiments in the past can be divided into two broad areas. First, there is the interest in stable hydrodynamics, the study of pressures obtainable, shock characteristics, and other aspects of the hydrodynamics problem as these affect various aspects of, for example, ICF, and how these experiments permit testing of hydrodynamics simulation capabilities.

Secondly, there is a keen interest in unstable hydrodynamic flow. This interest includes understanding flow in the linear growth regimes, through the non-linear regimes to turbulent flow. The instabilities that have been tackled include RayleighTaylor, Richtmeyer-Meskov, and Kelvin-Helmholtz. These experiments have attacked the unstable flow problem in a high-energy-density matter, anci that in itself may be a unique aspect of the work.

In addition, the dimensionality of the flow studied can be changed in the experiments. In preliminary experiments, such as those illustrated below, the desire is for simplicity, but this is a goal and is not always achieved. On the other hand, there are experiments in converging spherical geometry, required for ICF, as contrasted with more idealized planar geometry experiments performed for basic information. Examples of both stable and unstable hydrodynamics in spherical and planar geometry are shown below. 


\section{1) Stable Hydrodynamics}

\section{a) Planar Geometry}

A series of experiments has been performed to investigate the hydrodynamic motion of plasmas. The goal is to design an experiment where the hydrodynamics could be rigorously investigated without other complicating factors. In particular the desire is to avoid any problems arising from laser-plasma interactions or strength-of-material complications. Therefore, it is considered essential to deposit the initial energy in a welldefined manner. These considerations led to the use of a short pulse of penetrating $x$-rays to provide the initial heating for the samples.

The x-rays were produced by focusing one or more of the beams of Nova onto a thin gold foil approximately $2000 \AA$ thick. The laser pulse length was 1-ns long. It was found that about $10 \%$ of incident laser light was converted into gold $M$-band $x$-rays with a photon energy between 2 and $3 \mathrm{keV}$ (see the section on Absorption and Radiation Sources for more information on the $x$-ray sources).

To filter out $\mathrm{x}$-rays of lower photon energy, a $50-\mu \mathrm{m}$-thick plastic foil was placed at a distance of $200 \mu \mathrm{m}$ from the gold foil between the Au x-ray converter and the sample to be heated. At a distance of $1000 \mu \mathrm{m}$ away from the gold foil we placed a 3- $\mu \mathrm{m}$ aluminum foil. This foil absorbed approximately half of the incident gold M-band radiation.

The heating spectrum has been accurately characterized using the $x$-ray filtered diodes instrument at Nova. This is a ten-channel detector that uses absolutely calibrated filtered $x$-ray diodes. X-ray crystal spectrometers were also used to further spectrally characterize the radiation. With this arrangement an aluminum foil was heated to a few $\mathrm{eV}$. At these temperatures the $\mathrm{x}$-ray absorption coefficierts of the aluminum are very well-known. Thus, the energy deposition in the aluminum is well characterized. The schematic layout is shown in Fig. III.G.1.a-1.

The expansion of the aluminum foil was observed by using a time-gated $x$-ray imager. A 2000- $\mu \mathrm{m}$ tantalum foil was placed perpendicular to the direction of the motion at a distance of $\sim 1 \mathrm{~cm}$ from the aluminum foil, thus allowing observation across the foil. The Ta was illuminated by one of the Nova laser beams that had been smoothed by a random phase plate, and this produced a uniform $x$-ray backlighter source. A filter between the backlighter and the aluminum foil minimized heating of the sample foil due to the backlighter. Imaging was accomplished by using a 10- $\mu \mathrm{m}$ pinhole array and a gated microchannel plate $x$-ray detector with a total spatial resolution of $15 \mu \mathrm{m}$. On each experiment four images can be taken, with a temporal resolution of better than $100 \mathrm{ps}$. The images are typically taken at 1-ns intervals.

An example of the reduced data for one such image is shown in Fig. III.G.1.a-2. At approximately $5 \mathrm{~ns}$ the aluminum foil and the plastic foil begin to collide, which is the limiting time for this configuration. When the results of these experiments were compared with calculations, reasonable agreement was obtained, as was to be expected for such a simple geometry. One such comparison is shown in the accompanying figure. 


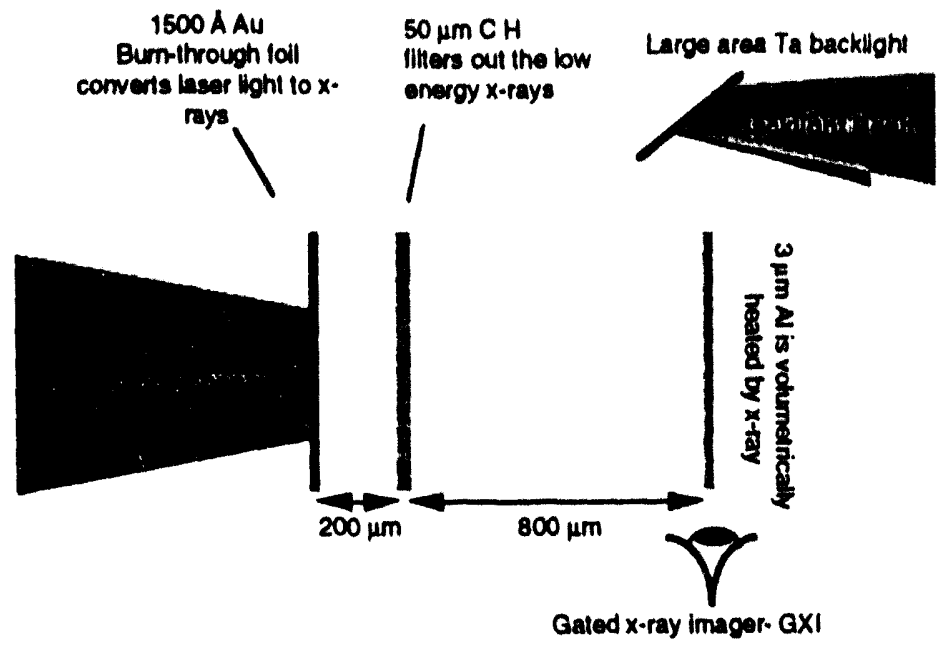

II.G.1.a-1) Schematic of experiments to provide a test bed for hydrodynamic simulations. The Al sample is uniformly heated by the X-rays created from a 5-beam illuminated Au burnthrough foil. The CH filter ensures that there is no radiation below the critical density for solid matter. The measurements are made using a multiple-framed $x$-ray imaging camera looking at the transmission of the large area backlight passing the target.

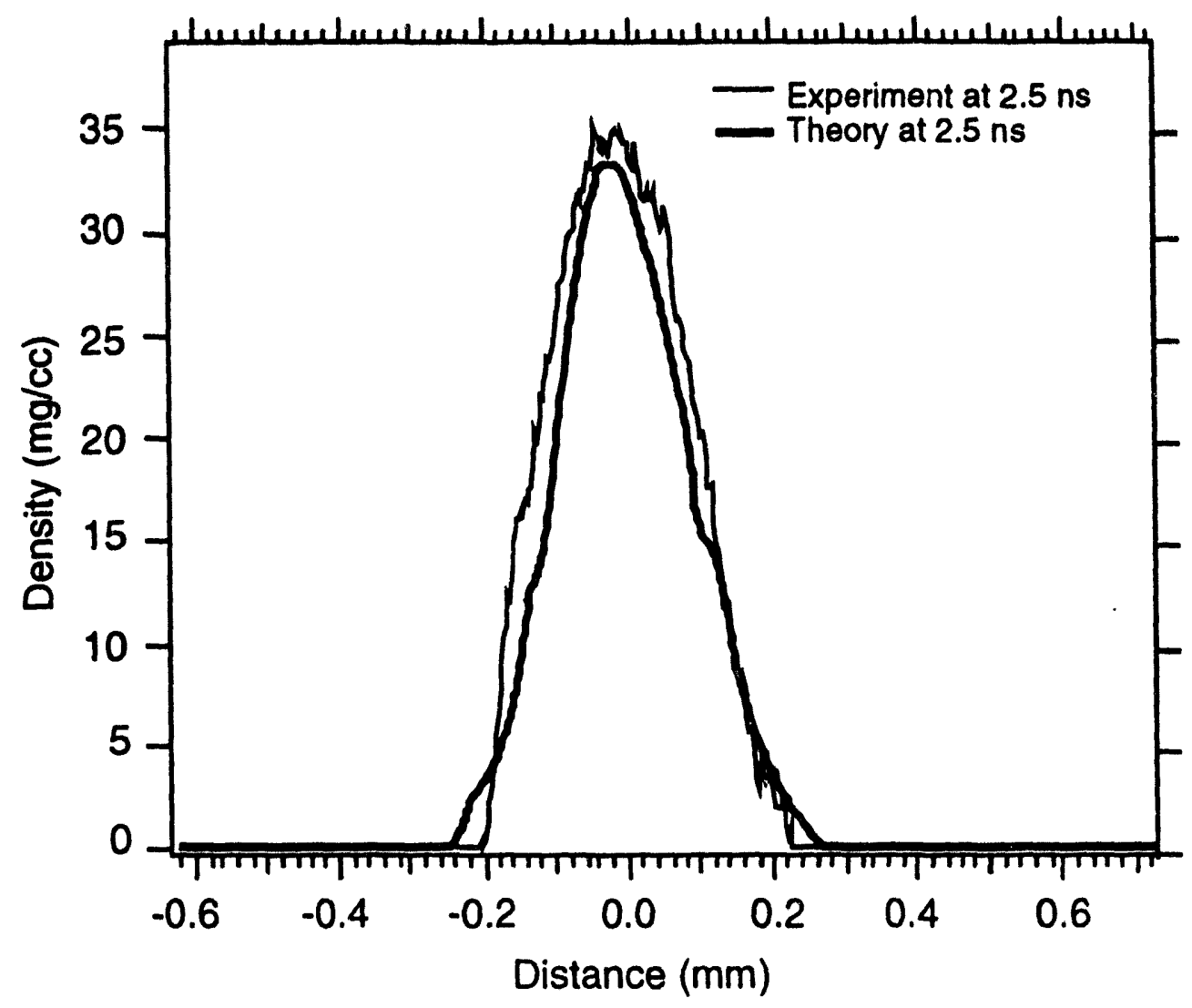

III.G.1.a-2) An example of the data obtained from the experimental test bed for hydrodynamic simulations. The theoretical prediction of the movement and expansion of the foil would be critical tests of the EOS and opacity of the hydrodynamic simulation. The agreement here is reasonable and would suggest more complicated experimental configurations could be tried. 
With these initial conditions verified, one can proceed to more complicated geometries, such as stepped targets, to provide further tests of the hydrodynamics.

\section{b) Spherical Geometry}

For the spherical geometry case, implosion imaging of ICF capsules, or microspheres, will be presented. A typical implosion sequence is viewed with a $5.3 \times$ magnification spanning $\Delta t=1.4$ to $1.95 \mathrm{~ns}$. The instrument used to make the measurement is a gated pinhole camera denoted WAX.

The active element of this multi-frame x-ray pinhole camera consists of a microchannel plate (MCP) overcoated with an S-shaped gold microstrip ${ }^{29}$ (see Fig. III.G.1.B-1). The MCP gain is switched on and off by a 170-ps FWHM 800-V pulse that travels along the serpentine strip. The WAX has been characterized in situ with short $x$-ray bursts produced by irradiating gold disk targets with tightly-focused, 100-J, 20-ps, $2 \omega_{0}$ Nova laser pulscis. The measured temporal resolution is $80 \pm 5 \mathrm{ps}$ FWHM, the voltage pulse propagation speed is $0.55 \mathrm{c}$, and the transverse sensitivity variations are $\leq 10 \%$.

The target consisted of a $470-\mu \mathrm{m}$ diameter polystyrene shell overcoated with a $55-\mu \mathrm{m}$ plastic ablator and filled with $50 \mathrm{~atm}$ deuterium, $0.1 \mathrm{~atm} \mathrm{Ar}$, and $0.02 \mathrm{~atm} \mathrm{Xe}$. This fill, as is indicated below, is used to provide a spectroscopic indicator of the implosion core composition. A 1-ns square, 17-kJ laser drive in a Au hohlraum was used. The interframe spacing is $45 \mathrm{ps}$, or $70 \mathrm{ps}$ between frames separated by a bend. The camera is filtered to pass emission only above $3.5 \mathrm{keV}$, at the Ar free-bound and Xe bound-bound photon emission energies. The capsule, which converges to $50 \mu \mathrm{m}$ diameter, already

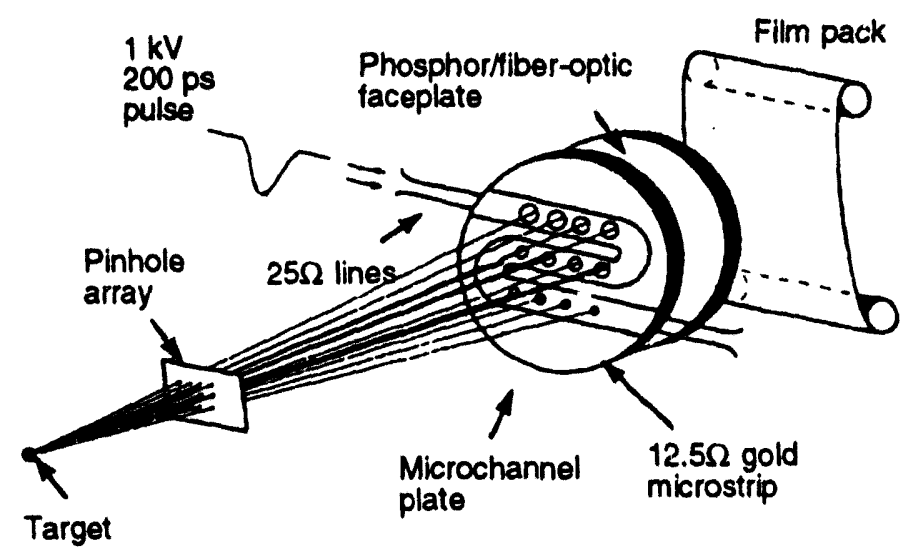

II.G.1.b-1) 12-frame X-ray gated imager, called the WAX. The instrument is capable of taking sequential images on a serpentine Au strip coated onto an MCP to make it sensitive to $x$-rays. Since a voltage is required to accelerate the electron produced to the rear of the MCP, one has a simple means of obtaining temporal resolution by the choice of voltage pulse shapes. 
appears in emission in frame 1 of Fig. III.G.1.b-2, $\Delta t=1.4 \mathrm{~ns}$, peaks in emission in the 5 th frame, $\Delta t=1.6 \mathrm{~ns}$, and is disassembling by frame $11, \Delta t=1.9 \mathrm{~ns}$. Residual diffuse $\mathrm{Au}$ $M$-band emission from the cooling hohlraum plasma is also observed through the $50 \%$ laser entrance hole, decreasing in intensity with delay, as would be expected after the end of the laser pulse.

Quantitative results are presented in Fig. III.G.1.b-3a and $-3 b$ for a similar shot with a higher drive of $29 \mathrm{~kJ}$. Time $=0 \mathrm{ps,}$ which is defined as peak emission time, occurs $0.3 \mathrm{~ns}$ after the laser pulse is switched off.

Figure III.G.1.b-3a shows the evolution of the FWHM of the emitting regions in two orthogonal planes. The $10 \% \mathrm{~m}=2$ asymmetry observed may be due to low- $\mathrm{Z}$ patches near the hohlraum equator creating a few percent lower flux at the target equator.

In Fig. III.G.1.b-3b, the evolution of the core central emission and hohlraum plasma emission are plotted. After deconvolving the instrument time response, the FWHM of the $x$-ray emission from the implosion is measured at $150 \mathrm{ps}$, in agreement with higher temporal resolution streaked spectral measurements.

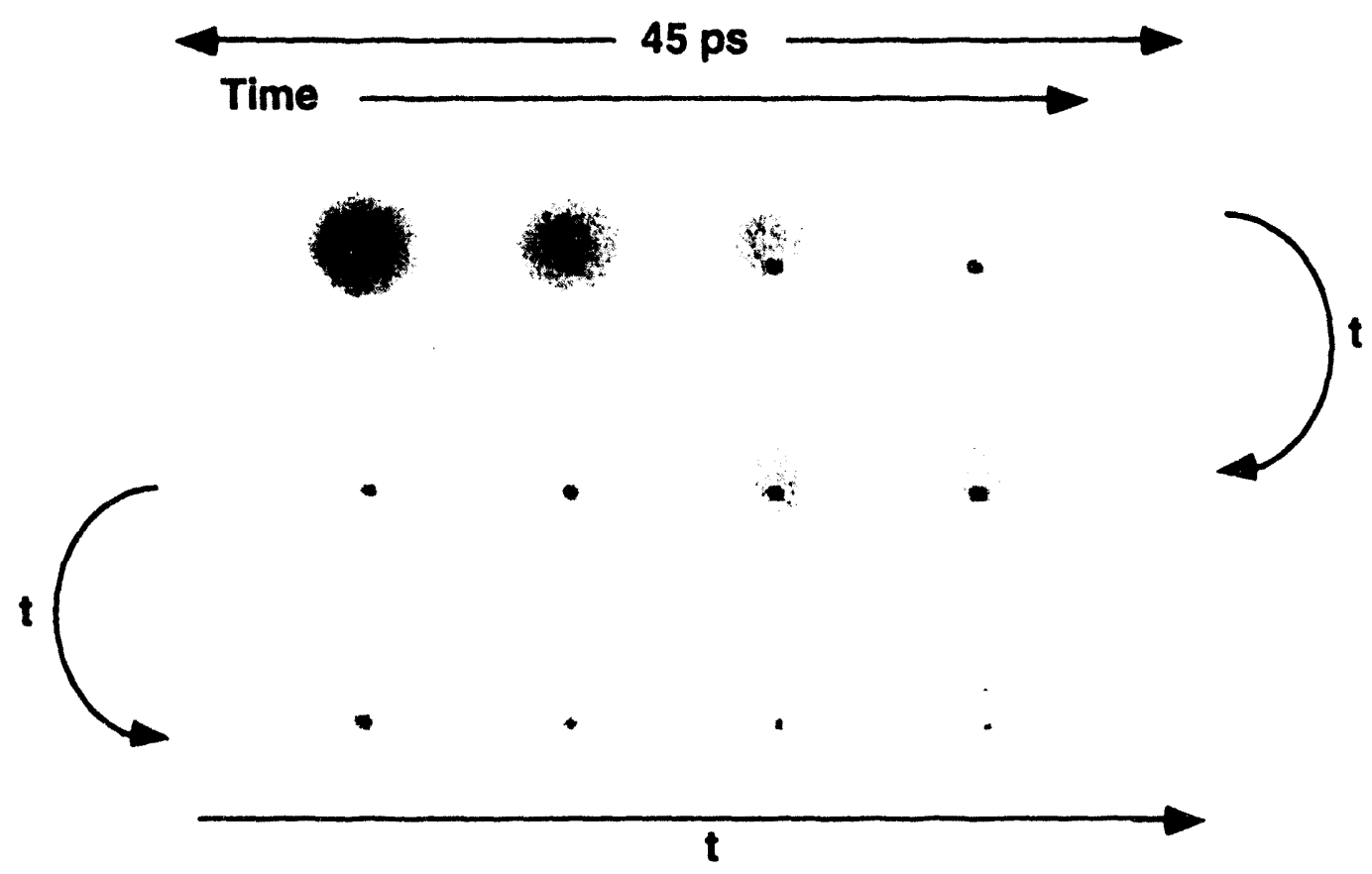

III.G.1.b-2) Typical implosion sequence 1-ns square, using the WAX diagnostic. The experiment had a $17-\mathrm{kJ}$ drive in scale-1 Au hohlraum. 

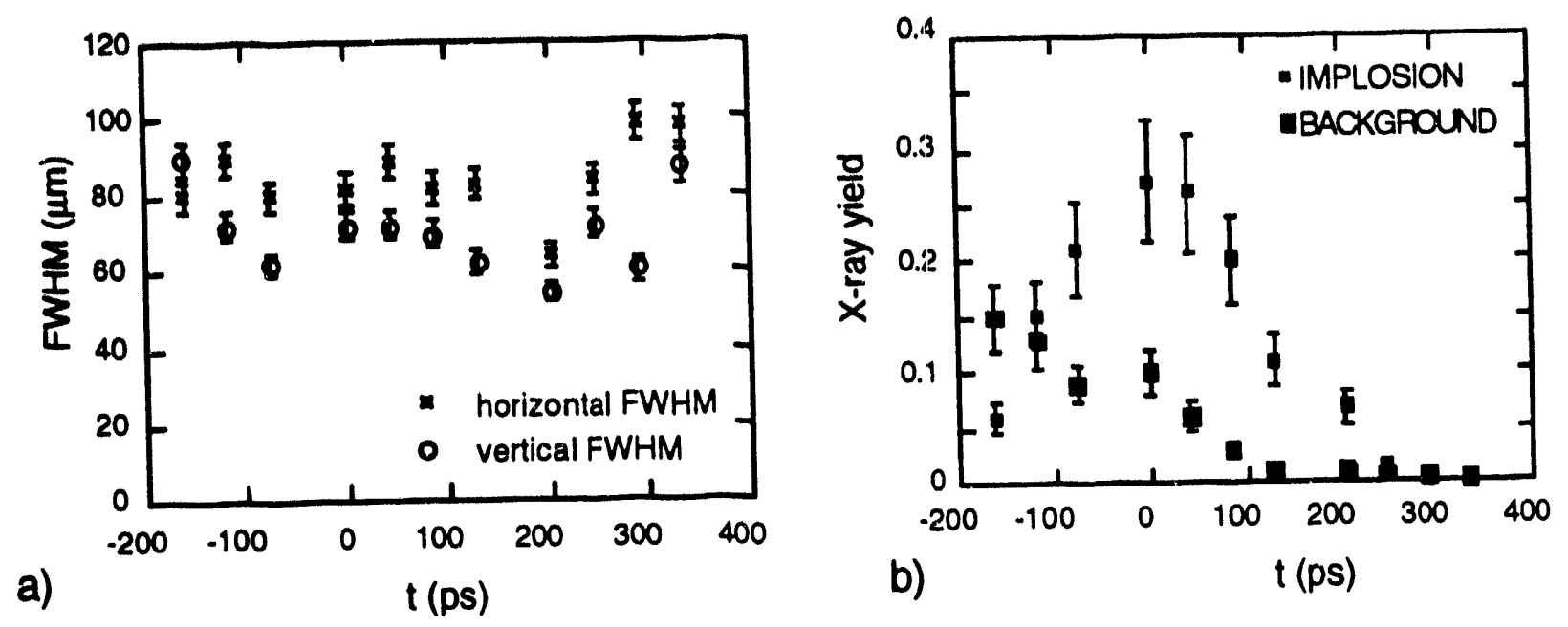

II.G.1.b-3) Time history of an implosion. a) Evolution of spatial FWHM of x-ray emission.

b) Evolution of target and hohlraum emission intensity. This experiment uses a 1-ns square, 29.kJ drive in scale-1 hohlraum.

\section{2) Unstable Hydrodynamics}

\section{a) Planar Geometry}

There are numerous possible areas of investigation for the study of planar instabilities in high-energy-density matter. The two that are discussed here should only be insidered a start on two aspects of the problem and not the defining experiments. In the first case we discuss the growth of an initially imposed perturbation under the influence of a continuous acceleration (i.e., the Rayleigh-Taylor case), while in the second case we treat an interface that has random perturbations and which is shocked (i.e., the Richtmeyer-Meshkov case).

First, an experiment is shown that is intended to study the large-growth RayleighTaylor instability using a radiation source as the driver. ${ }^{30}$ See Fig. III.G.2.a-1 for a schematic of the experiment. In these experiments, planar foils of fluorosilicone are accelerated by $x$-ray ablation. The foil trajectory is measured using edge-on radiography to check the bulk movement of the ablating sample.

In separate experiments, which employed face-on radiography, contrast in the optical depth is measured as a function of time, from which the evolution of the $50-\mu \mathrm{m}$ wavelength initially impressed sinusoidal perturbations can be deduced. The measurements of the growth of the perturbation were made using both an $x$-ray streak camera, which shows the continuous one-dimensional growth of the perturbation during the experiments, and a two-dimensional image with a frame time of $100 \mathrm{ps}$, showing the full foil used to ensure the planarity of the foil. 


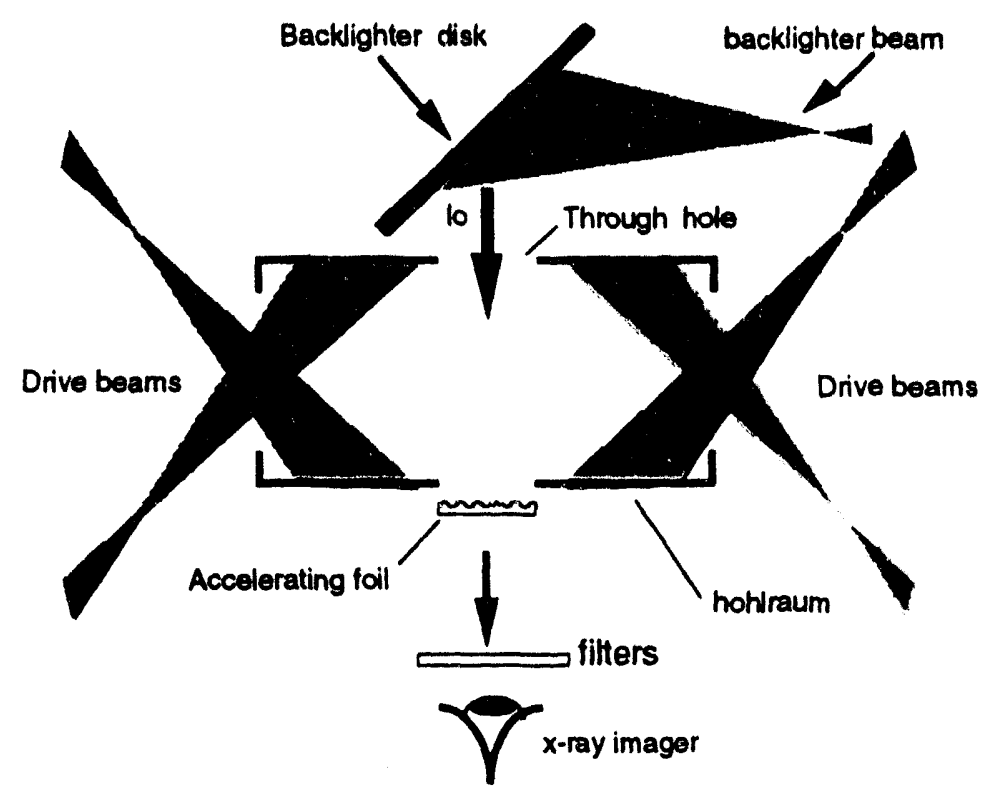

III.G.2.a-1) Schematic of the experimental setup, showing the face-on radiography of a target that has been impressed with a sinusoidal pattern.

In Fig. III.G.2.a-2a, one sees the streak camera image, with time in the vertical and position across the foil in the horizontal. The image shows that the optical depth modulation of the accelerated foil and indicates that the foil becomes, overall, increasingly bright due to thinning. In turn, the thinning is due to the formation of the "bubble-andspike" shape characteristic of the nonlinear Rayleigh-Taylor regime. Figure III.G.2.a-2b shows a two-dimensional image of the foil, taken at $2.6 \mathrm{~ns}$, for a frame duration of $100 \mathrm{ps}$. This data is essential to ensure that there is no transverse distortion of the foil.

The quantitative data is obtained by taking intensity traces at different times transverse to the grooved structure. In Fig. III.G.2.a-3 we show a sample of such a series of traces. Note that the curves, which represent different times in the evolution, are offset for ease of comparison. At early times the contrast is small and still sinusoidal, indicating that the Rayleigh-Taylor instability is in the linear regime. Late in time, the contrast is larger and distinctly non-sinusoidal, exhibiting the bubble-and-spike shape characteristic of the non-linear Rayleigh-Taylor regime. The rapid flattening of the modulations in the top two curves results from the burn-through when the bubbles break out of the back side of the foil. At this point, the spikes are still being ablated away, but no longer can be replenished by matter flowing down from the bubbles.

These experiments have been extended from the single mode example shown here, to multiple mode experiments, ${ }^{31}$ and to buried interfaces with imposed mode structures. ${ }^{32}$ The limiting case of a random set of perturbations at a buried interface requires a somewhat different technique. 

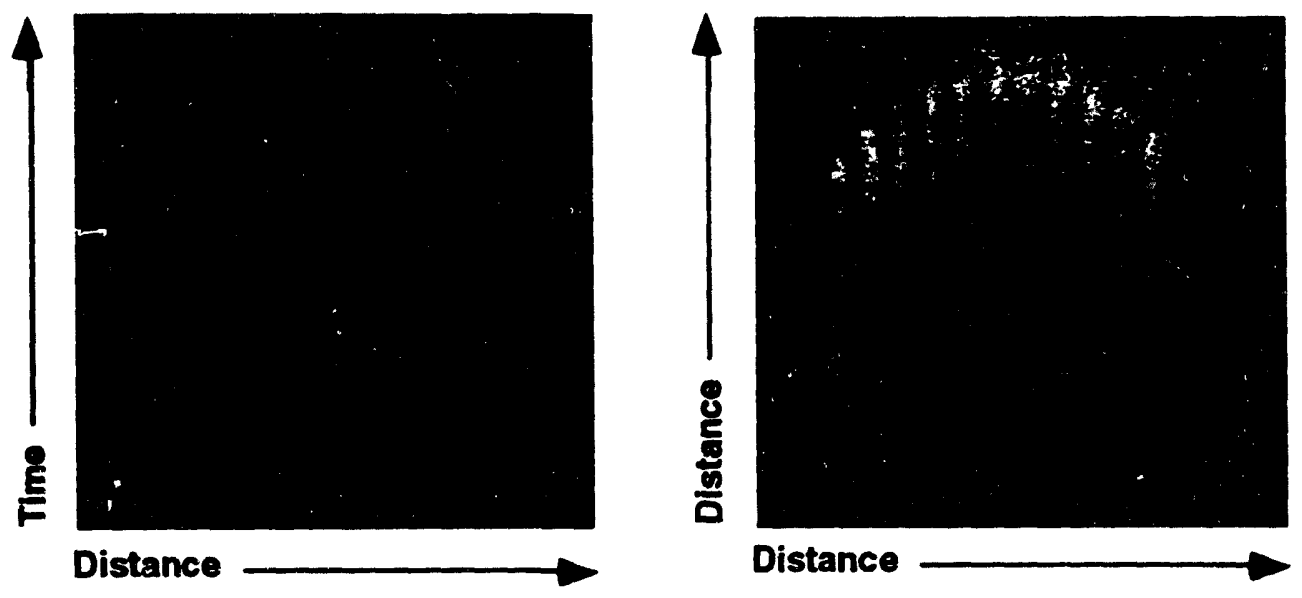

II.G.2.a-2) The face-on image of the backlight source absorbed by the foil with initial perturbation. a) Time increases in the vertical direction and position across the foil in the horizontal. The end of the bright spot occurs when the backlight ceases to radiate effectively. b) The faceon two-dimensional image of the foil indicating little, if any, distortion of the foil.

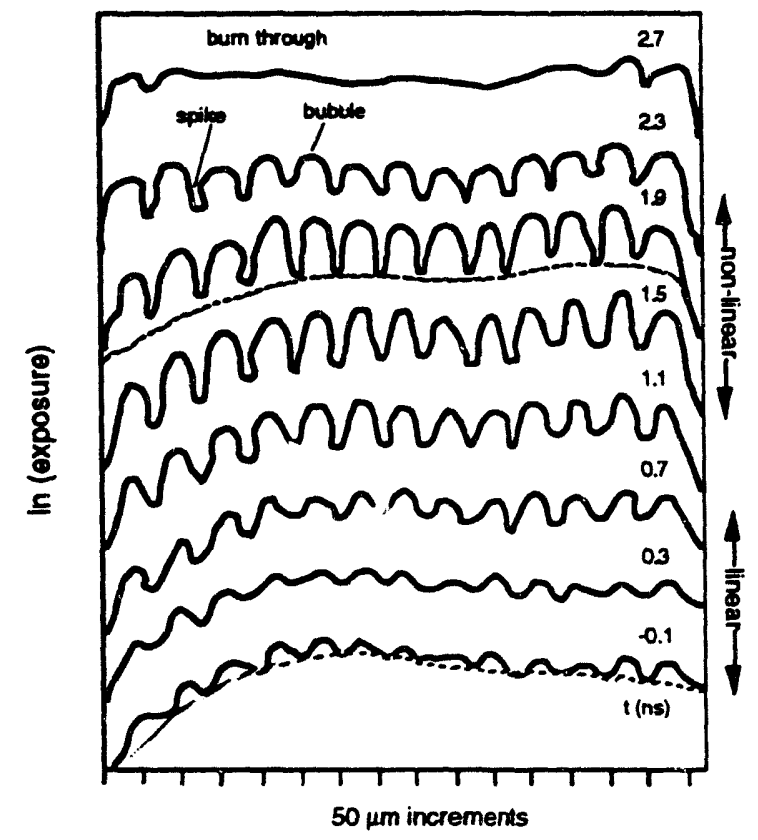

III.G.2.a-3) The traces for the accelerated foil with an initial perturbation of $50 \mu \mathrm{m}$ wavelength and $1.9 \mu \mathrm{m}$ amplitude sinusoidally imposed on the surface. The curves are offset in the vertical direction to allow simple comparison. The backlight intensity variation across the foil and as a function of time is noted by the dashed line at the initial time and later in the pulse. 
The second case we discuss under planar unstable flow concerns an imbedded interface with random perturbation and an implosive shock acceleration. Here the technique of point projection spectroscopy is used. A schematic of the experiment is illustrated in Fig. II.G.2.a-4a. A planar target with an ablator section and a foil section (shown in Fig. III.G.2.a-4a) is radiatively accelerated normal to its surface. The drive was produced by focusing 6-10 kJ of $0.35-\mu \mathrm{m}$ light in $1 \mathrm{~ns}$ from eight of the beams of Nova into a hohlraum. A planar shock of $\sim 60 \mathrm{Mbar}$ pressure with a rise time of less than $50 \mathrm{ps}$ propagated through the layered target, accelerating it to about half of its final velocity and driving an instability between the ablator and foil materials.

After a suitable delay the target was backlit by a flash of $x$-rays produced by focusing another beam, with $200 \mathrm{~J}$ of $0.35-\mu \mathrm{m}$ light in a pulse length of 100 to $200 \mathrm{ps,} \mathrm{onto} \mathrm{a}$ $22-\mathrm{mm}$ coated fiber. The fibers were coated with 1- $\mu \mathrm{m}$ thick cosputtered mixtures of $\mathrm{Au}$, $\mathrm{Bi}$, and $\mathrm{Pt}$, chosen to give bands of $\mathrm{x}$-ray emission in the 2-3-keV region. As this flash came from a small source, it was used to project a shadow of the target in motion onto $\mathbf{x}$-ray film after Bragg reflection from a potassium acid pthallate crystal. Since the crystal reflects only when Bragg's law is satisfied, the shadow has spectral as well as spatial resolution in one direction, the $x$ direction in Fig. III.G.2.a-4b. Spatial resolution is also obtained in the $z$ direction, normal to the disk, so that the $\mathrm{z}$ distance moved by various materials in the package could be measured.

The materials in the target are chosen to have photoabsorption edges in the wavelength range of the shadow of the package, e.g., the sulfur $\mathrm{K}$ and the Mo $\mathrm{L}_{\text {III }}$ and $\mathrm{L}_{\text {II }}$ shown in Fig. II.G.2.a-4c. The measured change in absorption across the edge and the known increase in opacity allow the areal density of the material to be evaluated. Mix of the target materials is detectable by the overlap, if any, of the photoabsorption edges schematically illustrated in Fig. III.G.2.a-4c for the no-mix, where there is minimal density mismatch at the interface, and for the mixed cases, where there is a density mismatch.

A serendipitous feature of this technique is that high opacity absorption lines form close to the cold edges as material is heated and ionized, acting as a sensitive, albeit qualitative, indicator of the position of the materials.

The accelerated target consisted of a 200- $\mu_{12}$ diameter, $\sim 1.5-\mu \mathrm{m}$ thick disk of Mo accelerated by a $60-\mu \mathrm{m}$ thick, lower density ablator of sulfinated plastic. Manufacturing resulted in a finish on the $\mathrm{Mo} /$ plastic interface of typically $0.1 \mu \mathrm{m} \mathrm{rms}$, with random wavelengths of 1-5 $\mu \mathrm{m}$. The high initial density mismatch in this experiment, measured by the Atwood number, was $\sim 0.8$ at the ablator-foil interface, derived from the density mismatch between the Mo, $10.4 \mathrm{~g} / \mathrm{cc}$, and the ablator plastic, $1.36 \mathrm{~g} / \mathrm{cc}$.

Most of the detailed information about the target is obtained from the absorption spectra of the materials in the target. Figure III.G.2.a-5 is an example of an image in which the target was backlit $7 \mathrm{~ns}$ from the start of the 1-ns drive. The image shows absorption as a function of wavelength and position in the horizontal $(x)$ direction, and as a function of position only in the vertical ( $z$ ) direction. The target image captured in flight can clearly be seen to have moved upward, away from the edge of its support. A 


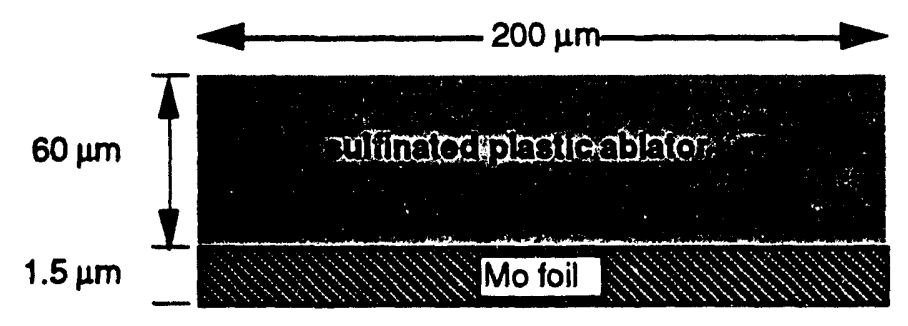

a) Target for studying mixing interfaces
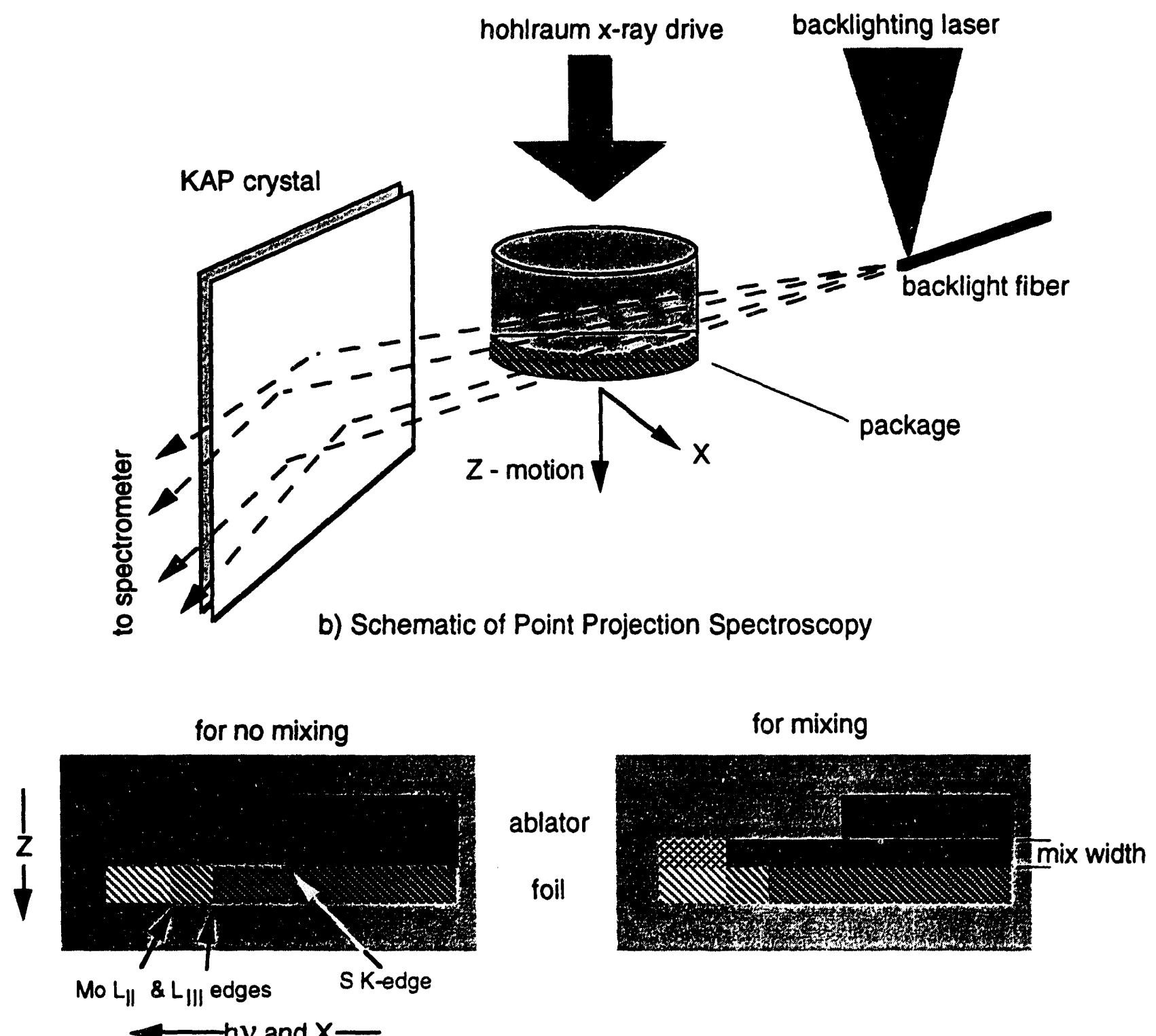

c) Spectrometer Images

III.G.2.a-4) A schematic of the point projection spectroscopy technique adapted for study of the mixing of interfaces. 


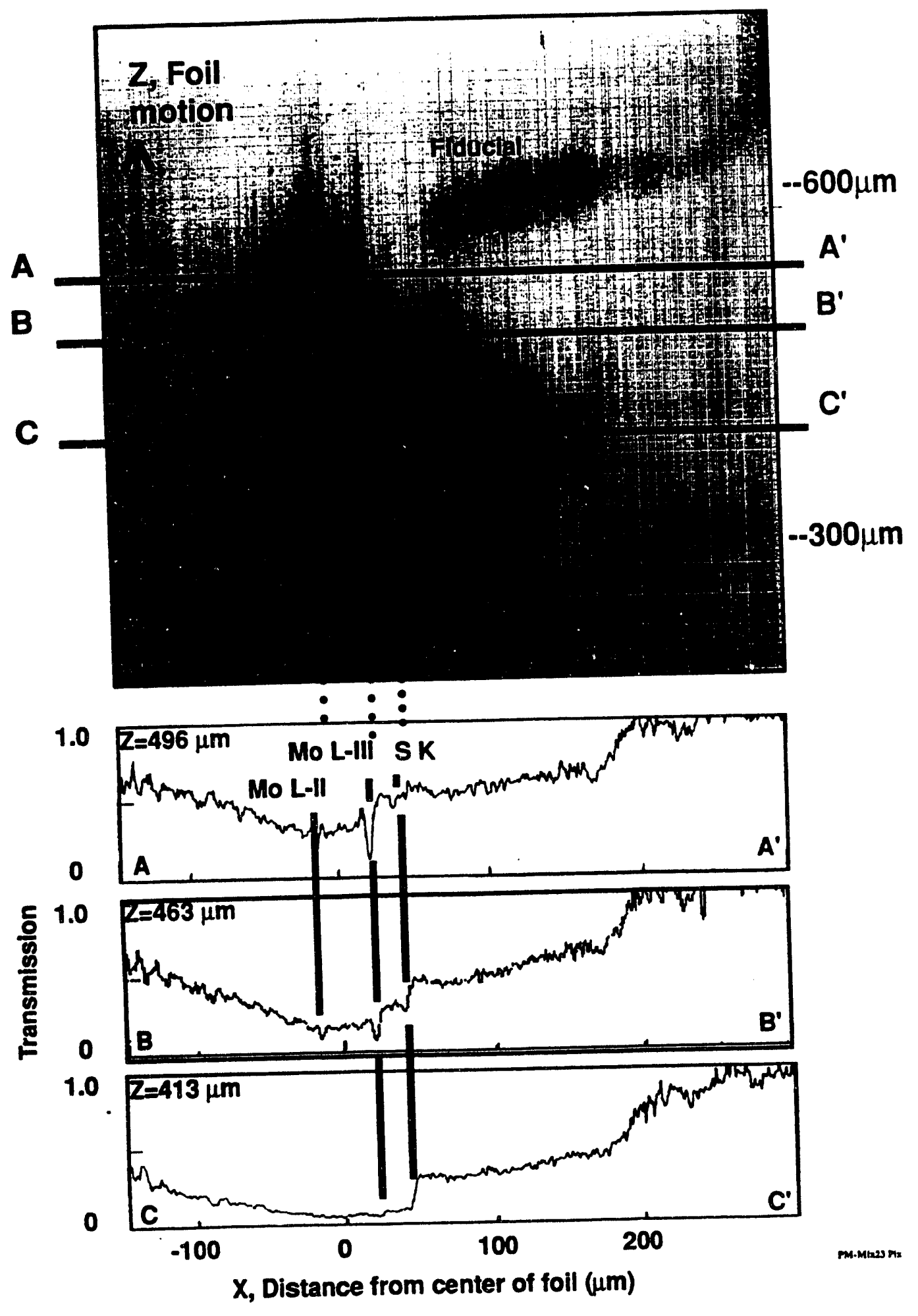

III.G.2.a-5) A radiograph of a sulfinated plastic ablator driving a Mo foil taken at $7 \mathrm{~ns}$ with a backlight pulse length of $150 \mathrm{ps}$, showing traces at three positions. Zero on the $\mathrm{z}$ vertical axis is the rear surface of the package at $t=0$, and on the $x$ axis zero is the original center of the foil. On the image the sulfur $K$ edge, the Mo LIII lines, and the edge of the package can be clearly seen. 
spatial fiducial made of $25-\mu \mathrm{m}$ wire provides a reference for the position of the package when the radiograph is taken. It als : demonstrates the $20-\mu \mathrm{m}$ source size which, when combined with the $10-\mu \mathrm{m}$ motional blurring, gives a limiting spatial resolution of $\sim 30 \mu \mathrm{m}$.

The traces at $z=413 \mu \mathrm{m}$ in Fig. III.G.2.a-5 show a clear sulfur K edge and a weaker Mo $L_{\text {III }}$ edge. The middle trace at $z=463 \mu \mathrm{m}$ shows both sulfur $K$ and Mo $L_{\text {III }}$ edges,

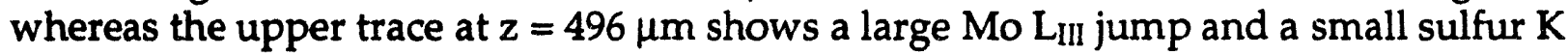
jump. These three traces demonstrate that the foil and the ablator are intermixed over a substantial spatial region.

The axial distribution of areal density of the materials of the package comes from the $\mathrm{K}$ and $\mathrm{L}_{\mathrm{III}}$ edge jump measurements exemplified in Fig. III.G.2.a-5. If $\mathrm{T}_{\mathrm{b}(\mathrm{a})}$ is the spectral transmission below (above) the edge then the areal density of the material along the viewing direction is given by

$$
\int_{\rho(l) d l}=\ln \left(T_{b} / T_{a}\right) / \Delta(\mu / \rho)
$$

where $\Delta(\mu / \rho)$ is the change in the mass absorption coefficient across a photo-absorption edge (2220-217 $\mathrm{cm}^{2} / \mathrm{g}$ and $1990-545 \mathrm{~cm}^{2} / \mathrm{g}$ for the sulfur $\mathrm{K}$ and Mo LIII edges, respectively) and $l$ is the viewing length.

From Fig. III.G.2.a-5, the diameter of the package at the observation time is $\sim 360 \mu \mathrm{m}$, which is larger than the initial 200- $\mu \mathrm{m}$ diameter because of decompression. Because the sulfur $\mathrm{K}$ and Mo LIII edges are close to the center of the package in Fig. III.G.2.a-5, this gives an upper limit on 1 of $360 \mu \mathrm{m}$.

These edge measurements of the axial distributions of areal density of $\mathrm{S}$ and Mo are shown in Fig. III.G.2.a-6a. The limitation on the lowest density measurable is $\sim 0.03 \mathrm{mg} /$ $\mathrm{cm}^{2}$ for the penetration of the sulfinated plastic into the Mo, and arises from errors in
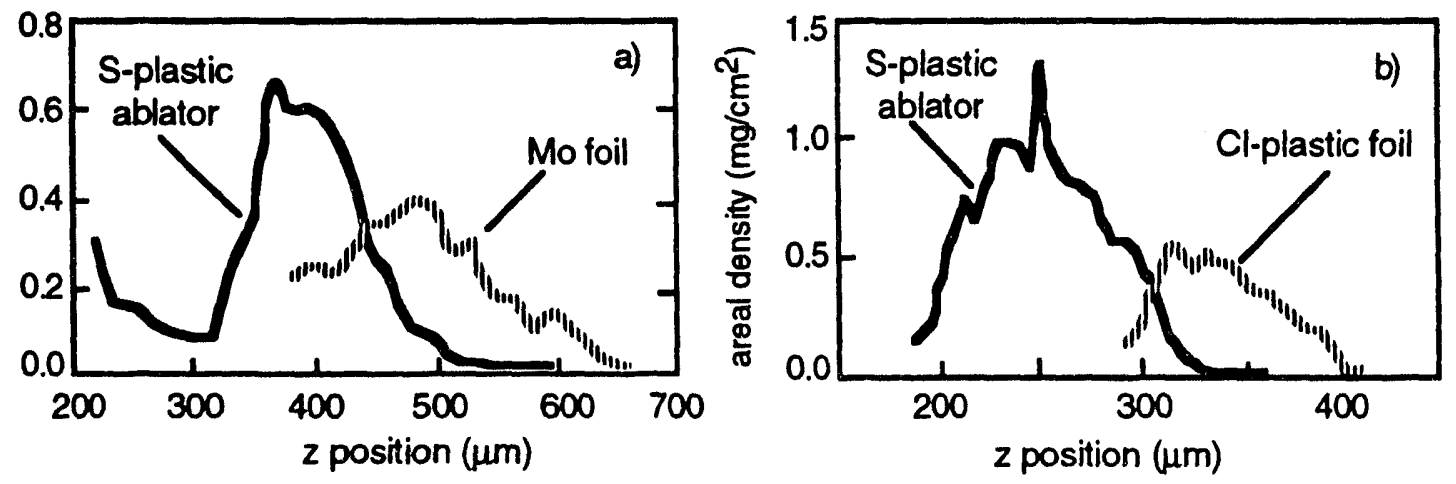

II.G.2.a-6) Axial distribution of mass areal densities for a) the high-mix case of Fig. III.G.2.a-5 and $b$ ) the null-mix shot measured at the package centers. The mass areal densities of the $S$ in the plastic, the $\mathrm{Mo}$, and the $\mathrm{Cl}$ in the plastic are plotted. 
the background, as the overall transmission is high $(\sim 0.7)$. For the Mo penetration into the sulfinated plastic, the lower limit on detectability is $0.2 \mathrm{mg} / \mathrm{cm}^{2}$ arising from the low overall transmission, $\sim 0.05$. Clearly an overlap region of $\sim 150 \mu \mathrm{m}$ exists that is much larger than the system resolution.

One potential problem in interpretation would be the loss of planarity, due either to bowing or tilting of the target. However, this apparent mixing of the ablator and the high-density foil is not due to bowing, as can be inferred from Fig. III.G.2.a-5, nor is it due to tilting, as another view at $44^{\circ}$ is used to check target orientation. Other effects, for example the ablator blowing by the Mo foil, could be producing the apparent mix, so to minimize this possibility control experiments were performed in which little mix should occur.

The target for these null shots had the same ablator as the high-mix shots, of density 1.36 , but a $15-\mu \mathrm{m}$ chlorinated plastic foil, polychlorostyrene of density 1.23 , replaced the Mo. The initial density mismatch is very small, having an Atwood number -0.05 , so little mixing is expected. The mixing of the foil and ablator in Fig. III.G.2.a-6b is less than $40 \mu \mathrm{m}$, which is approximately the experimental resolution.

\section{b) Spherical Geometry}

The most obvious method to study spherical unstable flow is to attempt to make the usually stable implosion unstable in a controllable way. To do this the use of perturbed microspheres "was perfected. A capsule shown in Fig. III.G.2.b-1 was a "bumpy" ball equipped with micron-size perturbations imposed on the outer plastic ablator surface, with a chlorine-doped pusher (i.e., the material at the interface with the gas core) and argon-doped 50-atm deuterium gas.

Fig. III.G.2.b-2 shows a single $12.5 \times$ magnification, $12-\mu \mathrm{m}$ resolution image of an implosion, using the bumpy ball, taken 0.2 ns after peak emission, taken by the WAX instrument discussed in Section III.G.1.b. Note that in the figure the positions of the

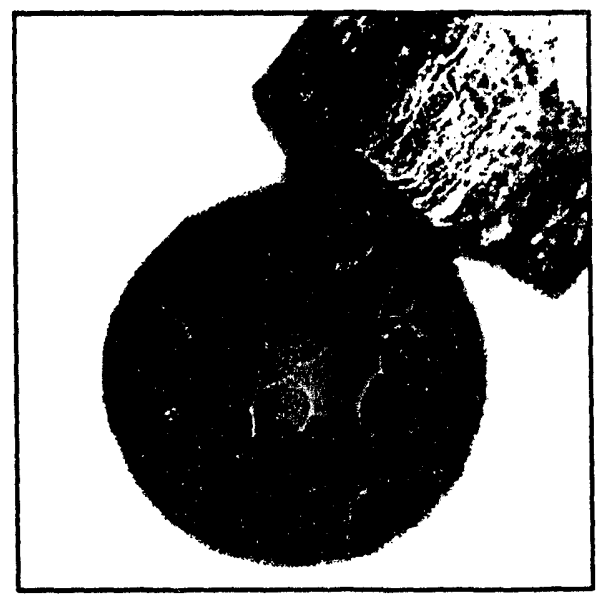

III.G.2.b-1) The machined "bumpy" ball. The bumps are random and are placed on the surface of the microsphere by laser irradiation. Note that the cylindrical object to the upper right side is there to hold the microsphere for processing. 


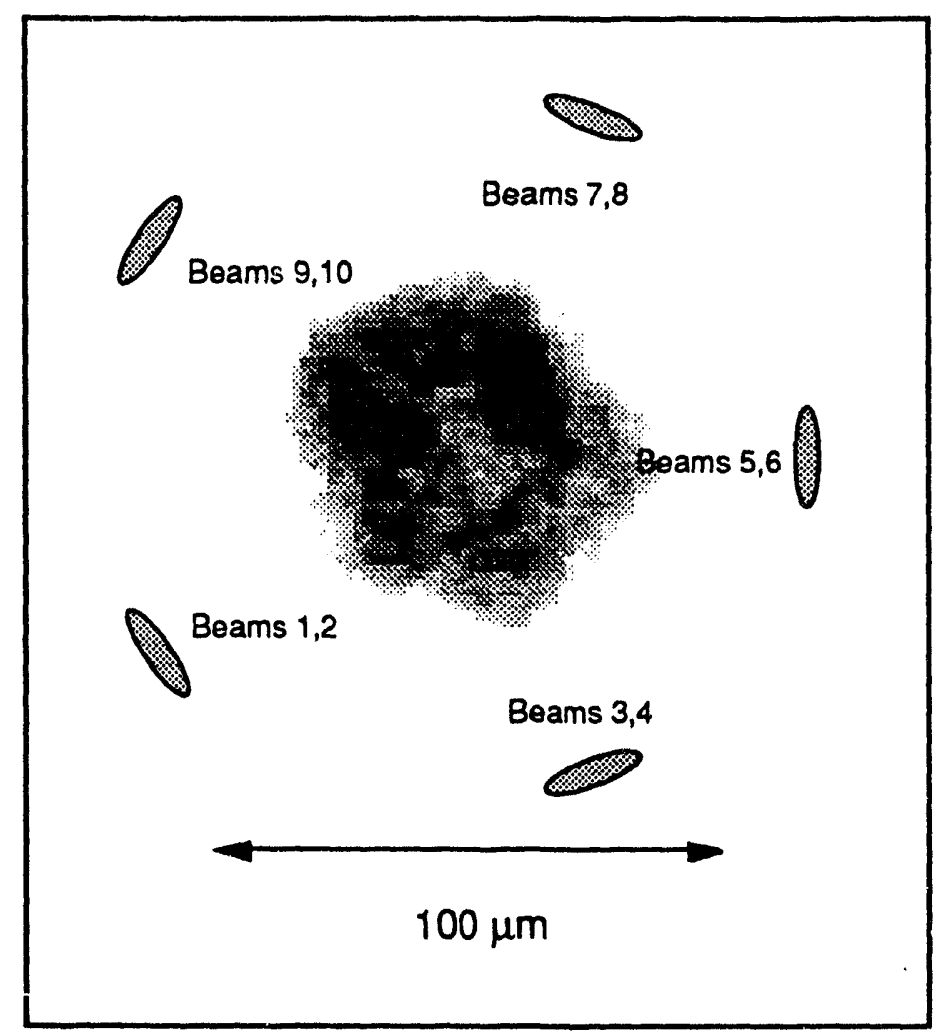

III.G.2.b-2) Expanded view of an implosion image taken using the WAX, showing an $\mathrm{m}=\mathbf{5}$ asymmetry and limb brightening.

beams on the hohlraum wall relative to the image are shown. The experiment used 1-ns square, $17-\mathrm{kJ}$ laser drive in a $2550-\mu \mathrm{m}$-long Au hohlraum. A concave pentagonal shape, the $m=5$ mode, is clearly observed. The nodes, corresponding to regions of lower flux, occur between laser spots. The $m=5$ symmetry is observed on almost all 1-ns square implosions.

In addition, Fig. III.G.2.b-2 shows evidence of higher emission from the edges of the capsule than from the center, commonly defined as limb brightening. A measured polar-averaged radial trace in Fig. III.G.2.b-3 shows the analyzed data that suggests that the evolution of the pusher-gas interface can serve as a measure of the degree of mix of the gas and the pusher, and might be diagnosed by time-resolved limb imaging.

As a further monitor of the mixing of the interface between the solid-density pusher and the gas fill in an implosion, the doping of the pusher with a high- $\mathrm{Z}$ material can both provide information on the pusher conditions and act as a diagnostic for mixing into the gas region. ${ }^{33}$ Using the same type of bumpy ball as shown in Fig. III.G.2.b-1, observations of the emission from various dopants included into the innermost region of the pusher have been made. Fig. III.G.2.b-4 shows $x$-ray streak camera data from both smooth and bumpy microspheres with dopant in the pusher that is $1 \% \mathrm{Cl}$ by number. The microsphere with the bumpy surface has significantly brighter $\mathrm{Cl}$ emission and weaker Ar emission than the microsphere with the smooth surface. This is an indication that some of the material has been mixed into the hot gas. 


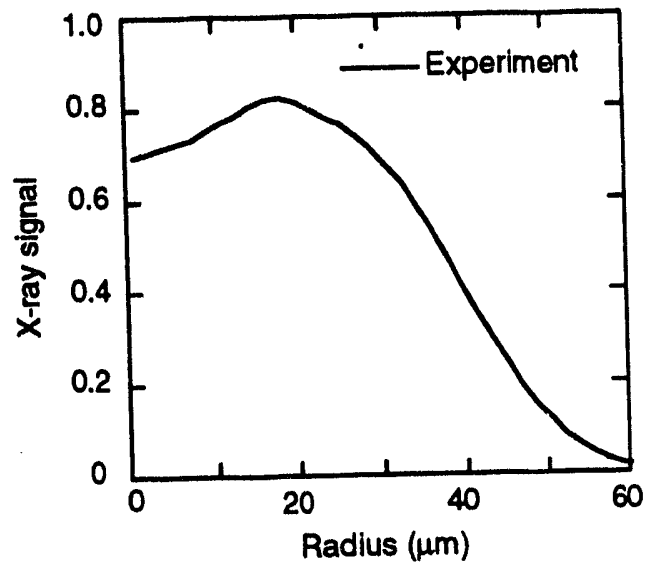

III.G.2.b-3) Measured trace quantifying the limb brightening. The comparison is only qualitative since absolute timing is not known and the target surface roughness is not fully characterized.

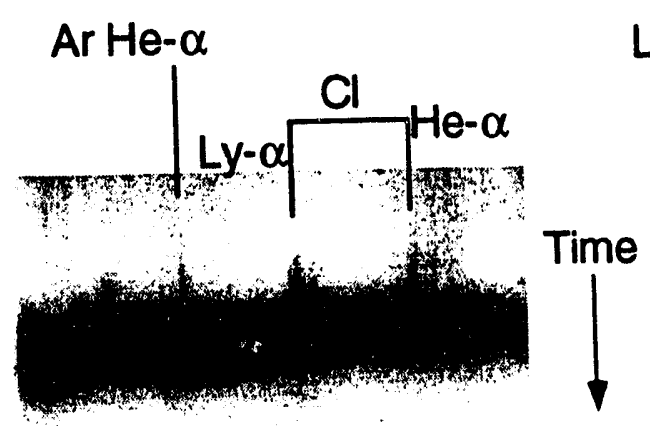

Bumpy

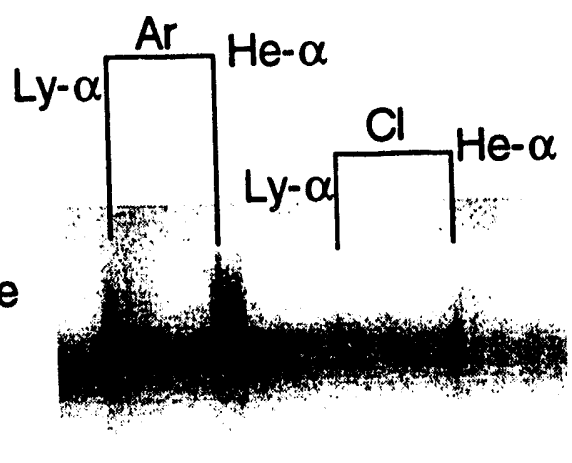

Smooth

III.G.2.b-4) Streak camera records of spectra from microsphere radiatively driven in a hohlraum. The $\mathrm{Cl}$ is doped into the pusher and the $\mathrm{Ar}$ is mixed into the gas core. The left hand spectrum shows the bumpy surface experiment and the right hand side shows the smooth surfaced experiments.

Further extensions of the technique for inferring mix into gas cores permits higher- $Z$ K-shell emitters to be used, as these spectra are simpler to interpret. Advances in target fabrication have expanded the choices of possible pusher dopant to include several metals. Figure III.G.2.b-5 shows the results of experiments with and without bumpy surface finishes. In the figure the microsphere with imposed bumps is shown to have a substantial He-like Fe $1 s^{2}-1$ s $2 p$ emission feature, while the smooth-surfaced microsphere shows no evidence of the Fe emission. Finally, there have been further improvements in the technique by using complex spectral features, for example UTAs, ${ }^{34}$ which, however, awaits theoretical further development. 


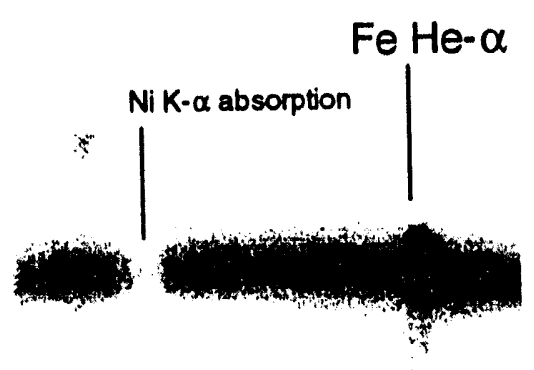

Bumpy

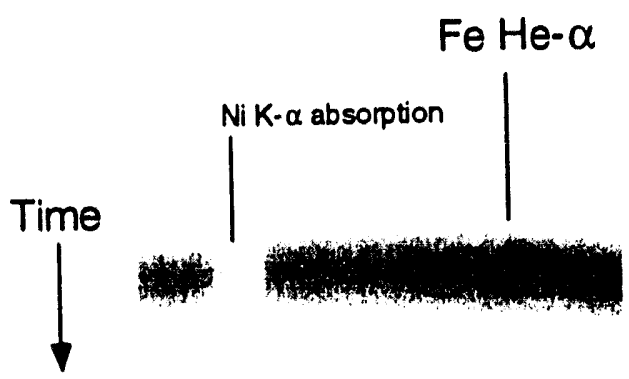

Smooth

III.G.2.b-5) Streak camera records of spectra from a microsphere radiatively driven in a hohlraum. The Fe is doped into the pusher and the Ar is mixed into the gas core. The left-hand spectrum shows the bumpy-surface experiment, and the right-hand side shows the smoothsurfaced experiments.

\section{H) Absorption and Radiation Sources}

The development of light-source capabilities will be broken down into three generic areas. First, there is the effort to use the high-energy laser to make spectrally continuous or quasi-continuous sources for use as absorption sources and as heating sources for the application of radiatively heating matter. Second is the work on the conversion of the high-energy laser into narrow-band radiation sources. Here the effort is usually quantified by the conversion efficiency into a single-line transition or into a small, closely grouped set of lines. Finally, there is the effort to further develop the $x$-ray laser, which work, of course, overlaps with the applications of x-ray lasers.

\section{1) Spectrally Continuous Sources}

There are two broad categories of quasi-continuous sources that one can identify. First there is the radiation source generated by a hohlraum and used to heat a sample either inside the hohlraum, as in the case of an implosion microsphere or an opacity sample, or abutted to it, as with the radiation flow experiments or the hydrodynamic mixing interface experiments. With this we group the Au burn-through foils used to heat the non-LTE tamped samples. Second, we have the effort to develop backlights that are quasi-continuous.

In the first, category the work done on the Au will be used to illustrate the cogent features. These burn-through foils have been studied in detail and provide a good sense of what to expect from very similar processes that occur in a hohlraum, noting, of course, that in a hohlraum one is using the front surface emission and an enclosure that will increase the energy in the radiation field. 


\section{a) Conversion Efficiency of Au Burn-Through Foils}

The conversion efficiency from visible laser light to $x$-rays can be roughly quoted as being in the region of $\geq 40 \%$ of the laser energy on target, noting that many papers use a conversion efficiency of $\mathrm{x}$-ray energy to absorbed laser energy. We choose the former because it gives a better idea of the x-rays available from a particular laser.

In Fig. III.H.1.a-1 we note the conversion efficiency from the front and rear side of gold, $A u$, foils of varying thicknesses from $500 \AA$ to $10,000 \AA$, for the two intensities of 1012 and $1013 \mathrm{~W} / \mathrm{cm}^{2} .35$ The figure shows the conversion efficiency observing from the front as the data points rising with increased thickness, and the rear as data points falling with increasing foil thickness. The limiting case of a solid target is approximated by a $10,000-\AA$ foil, where the conversion efficiency transmitted in $x$-rays is very small, while the front side conversion rises to $\sim 50 \%$ or more. Note that these data have been obtained both with filtered $\mathrm{x}$-ray diode arrays and with spectrometers, and both data sets are shown.

The figure indicates two important points. First, the conversion efficiency is not strongly dependent on intensity above $1012 \mathrm{~W} / \mathrm{cm}^{2}$, and second, the thinnest foils convert less. The reason for this is obvious when one looks into the transmission of laser

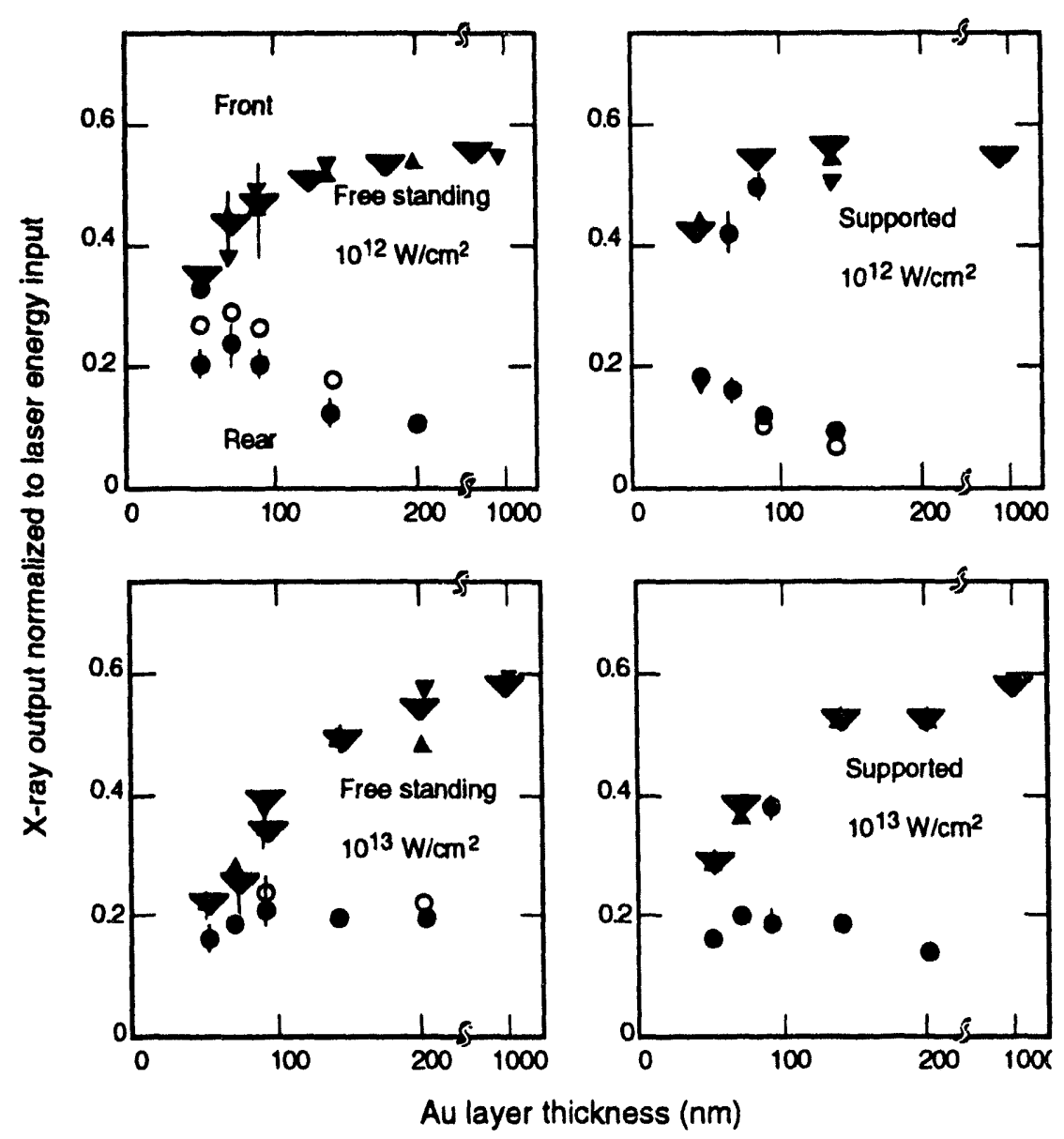

III.H.1.a-1) Total x-ray emission measured as conversion efriciency vs target thickness, from the front and rear surfaces of free-standing and supported Au targets. The irradiation is $10^{12}$ and $1013 \mathrm{~W} / \mathrm{cm}^{2}$. The front surface always has a larger conversion efficiency than the rear. Note that the method of support and the intensity do not affect the results. 
energy through the foil. This is presented in Fig. III.H.1.a-2, where the ratio of the transmitted-to-incident laser energy is shown. Note that in the figure the higher intensity shows more burn-through, but that the fraction is less than or equal to 0.01 for $1500-\AA$ foils. The reason for this burn-through is due to inhomogeneities-hot spots or intensity fluctuations-in the beam profile. These hot spots can be smoothed out and, indeed, have been in more recent experiments.

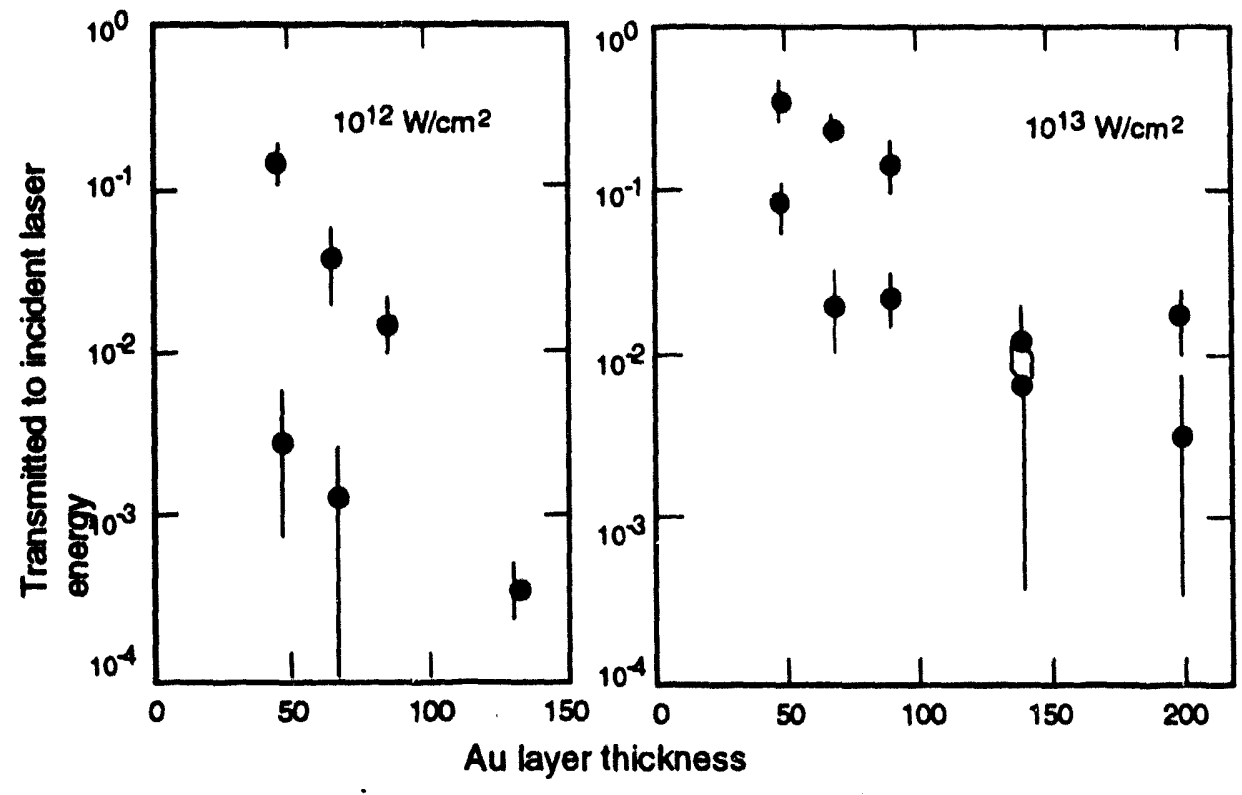

III.H.1.a-2) The measurement of the transmitted laser as a fraction of the incident laser energy vs target thickness for two different intensities. This is from the same experimental setup used to obtain the data in Fig. III.H.1.a-1.

b) Spectral Character of Au Burn-Through Foils

The spectral character of the emission found from such a Au target is shown in Fig. III.H.1.b-1. ${ }^{36}$ Here the time-dependent spectrum is shown vs energy. The spectrum was taken from the rear side of a Au foil that was $1000 \AA$ thick, mounted on a $5000-\AA$ layer of plastic, $\mathrm{CH}$. The front and back spectra of a target of this thickness will be very similar, with no major differences in the spectral character. The location of the two "bumps" in the spectrum correspond to the $\mathrm{O}$ and $\mathrm{N}$ bands, at $\sim 300 \mathrm{eV}$ and $\sim 800 \mathrm{eV}$ respectively.

There is also an M-band contribution which does not show up on soft x-ray instruments because it spans the energies between $2000 \mathrm{eV}$ and $3600 \mathrm{eV}$. In Fig. III.H.1.b-2 the M-band contribution is shown for a Au disk irradiated at $2 \times 1014 \mathrm{~W} / \mathrm{cm}^{2} .{ }^{37}$ The units are unique to the ICF program at LLNL and can be easily converted. The line drawn on the figure indicates the spectral region over which the M-band contribution is defined. 

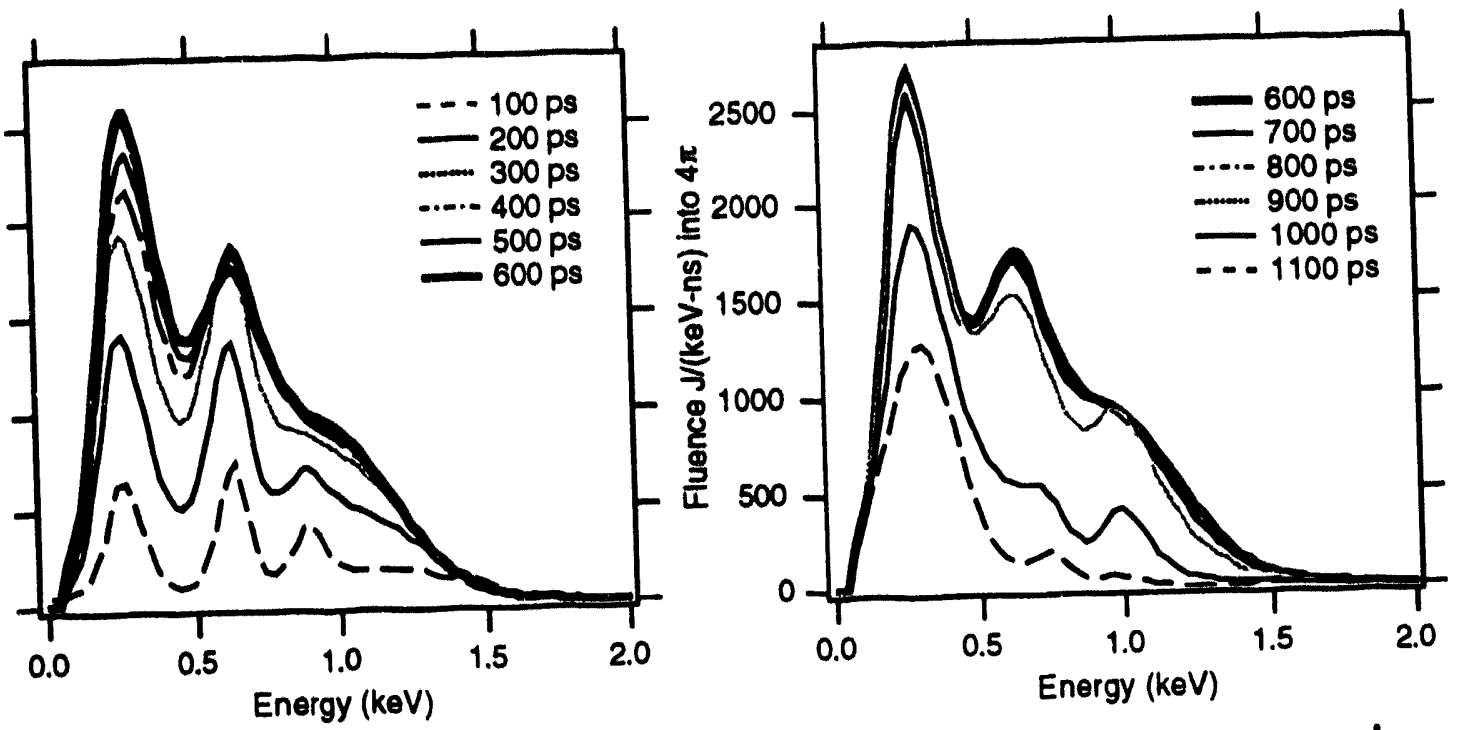

II.H.1.b-1) The time history of the spectrum vs energy in $\mathrm{eV}$ from the rear side of a 1000- $\AA$ gold foil supported on a 5000- $\AA$ CH layer irradiated at $2 \times 10^{14} \mathrm{~W} / \mathrm{cm}^{2}$. The results are measured by a 12-channel filtered $x$-ray diode which covers the range from $80 \mathrm{eV}$ through $2000 \mathrm{eV}$.

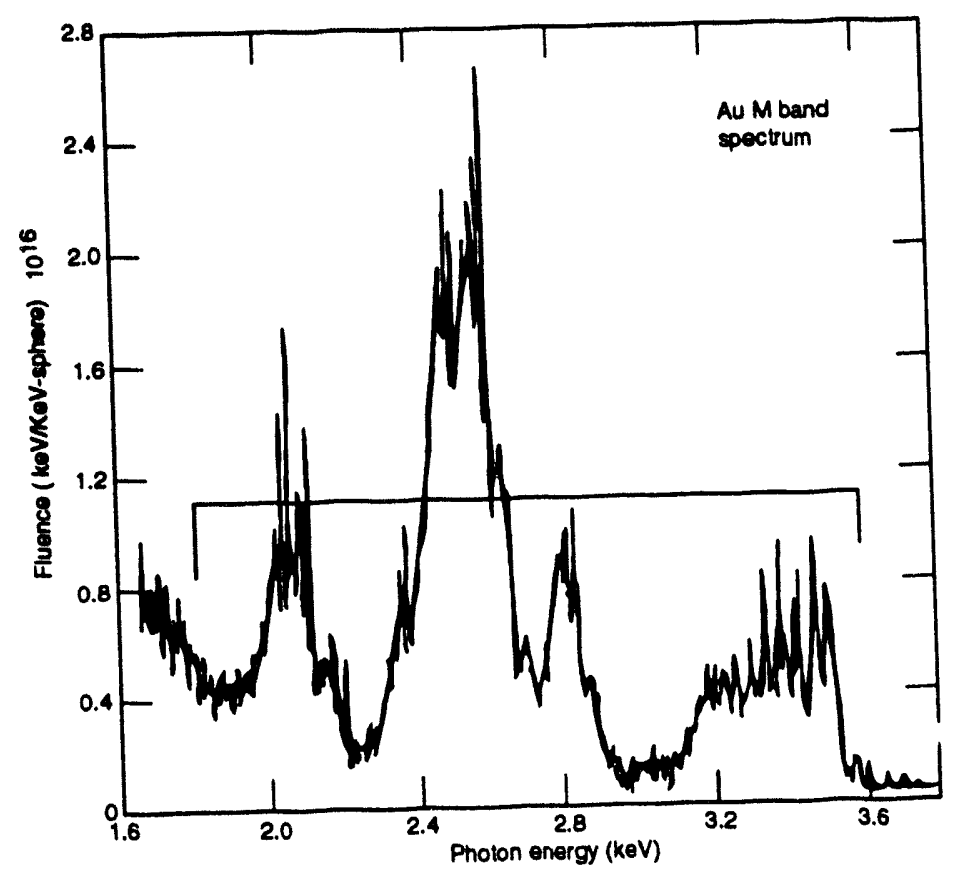

III.H.1.b-2) The spectrum of the Au M-bands which cover the spectral region from 2000 to $3500 \mathrm{eV}$ from a thick target with intensities of $4 \times 10^{14} \mathrm{~W} / \mathrm{cm}^{2}$. This bandlike structure is obtained for all high-Z elements. 


\section{c) Temporal Behavior of Au Burn-Through Foils}

The time history of the emission in the harder $\mathrm{x}$-ray regimes, such as the M-band, will follow the laser pulse, and there is much data to support this supposition. However, the soft $x$-ray spectrum will last for time longer than the laser pulse, and the duration will increase as the wavelength of the radiation increases. Thus, for the softest $x$-rays measured using the filtered $x$-ray diodes (i.e., $\sim 80 \mathrm{eV}$ ) in Fig. III.H.1.b-1, the time duration will be two to three times the pulse duration. Because the pulse is $1 \mathrm{~ns}$ in duration, this indicates emission on the order of several ns.

In Fig. II.H.1.c-1, the total energy emitted per unit time from a source on the rear of Au foil is shown. The normalization is made to a sphere (i.e., "into $4 \pi$ ") but the measurements reflect only the intensity of a specific ray. Thus, the correct units should be joules per ns per steradian, and the fact that these sources tend to look Lambertian (i.e., they have $\cos (\mathrm{q})$ dependence) provides the correct intensity. Here $\mathrm{q}$ is the angle of observation relative to the target surface normal.

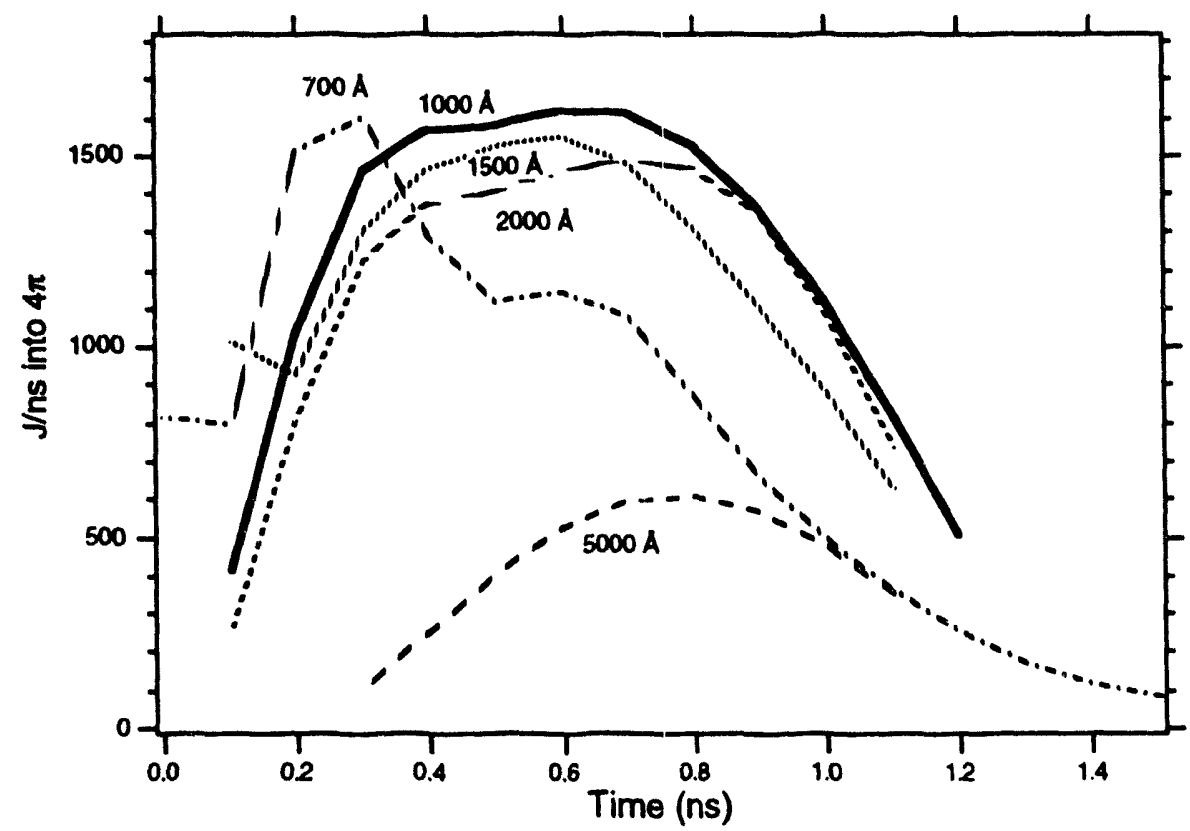

III.H.1.c-1) The temporal history of the spectrally integrated emission from the rear side of Au foils for various foil thicknesses irradiated at $2 \times 10^{14} \mathrm{~W} / \mathrm{cm}^{2}$. Note that the duration of the laser, $1 \mathrm{~ns}$ in this case, is not closely followed by the softer $x$-ray emission. The time delay for the thicker foils occurs due to the lag in getting the softer $x$-ray to the back surface. It is noted that the hard $x$-ray flux (e.g., the Au M-band contribution) shows no timing differences with foil thickness. 


\section{d) Spectral Character of Other Foils}

Next we illustrate the fact that the spectral character of the soft x-ray emission can be modified by the choice of target material. ${ }^{38}$ In Fig. III.H.1.d-1a we show the soft $x$-ray spectrum from pure targets of several elements from $Z=56$ to 63 , while in Fig. III.H.1.d-1b we show the spectrum from targets where there is a compound which contains approximately $1 \%$ of the element of interest. The spectrum in each case shows that the opacity of the sample increases the softer $x$-ray feature and thereby increases the quasi-continuous nature of the emission. Moreover, the ability to tailor the source in energy can be observed.
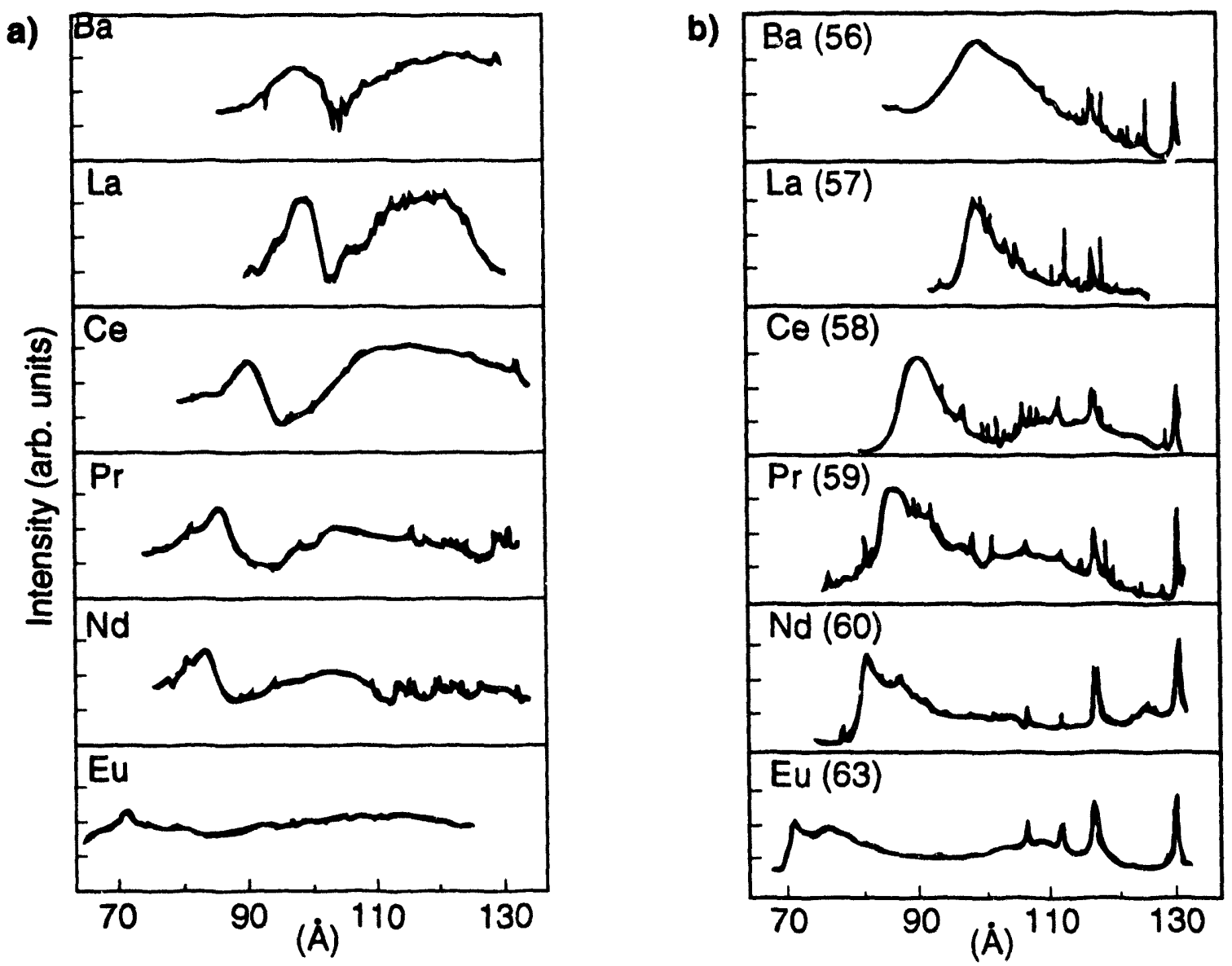

III.H.1.d-1) The spectral character of various elements from $Z=56$ to 63 . In cases (a) the targets are made of the pure element, while in cases (b) the element is a $1 \%$ impurity. The increased optical depth in cases (a) leads to an enhancement of the low-energy features compared to cases (b), where the optical depth is low and the evidence of individual lines becomes apparent. Further note that the dominant features move toward higher energy as $\mathbf{Z}$ increases. 
In Fig. III.H.1.d-2 we show the same type of data, but here for a much wider range of $Z^{\prime} s^{39}$ The figure shows the front surface spectra measured in 1012 ergs per $\mathrm{cm}$ per steradian for an irradiance of $3 \times 10^{13} \mathrm{~W} / \mathrm{cm}^{2}$ using the frequency-doubled laser at $0.53 \mu \mathrm{m}$. Note that the $\mathrm{Au}, \mathrm{O}$, and $\mathrm{N}$ bands are seen while the $\mathrm{M}$ band is beyond the spectral coverage. In Fig. III.H.1.d-3 the absolute conversion efficiency of the x-ray production is presented as a function of target $Z$. The net increase in the $x$-ray conversion is quite marked for the high- $Z$ elements.
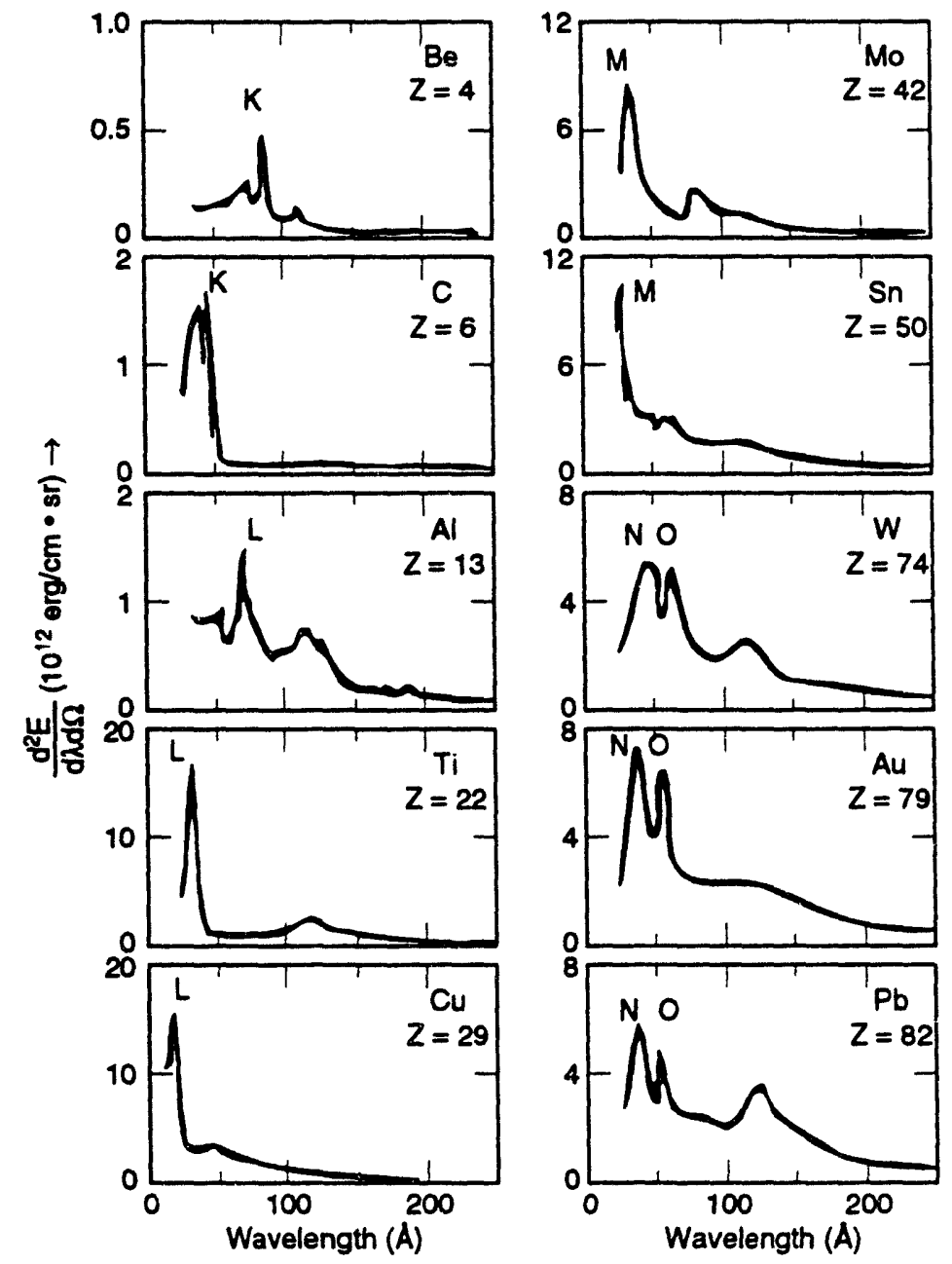

II.H.1.d-2) Absolutely measured spectra emitted by a laser irradiated target of different elements. The intensity is $3 \times 1013 \mathrm{~W} / \mathrm{cm}^{2}$ the pulse duration is $3 \mathrm{~ns}$ and the wavelength of the laser is $0.53 \mu \mathrm{m}$. The spectroscopic structure $K, L, M, N$, and $O$ refer to the shells from which the emission arises. Note that the intensity axis changes in the various plots. 


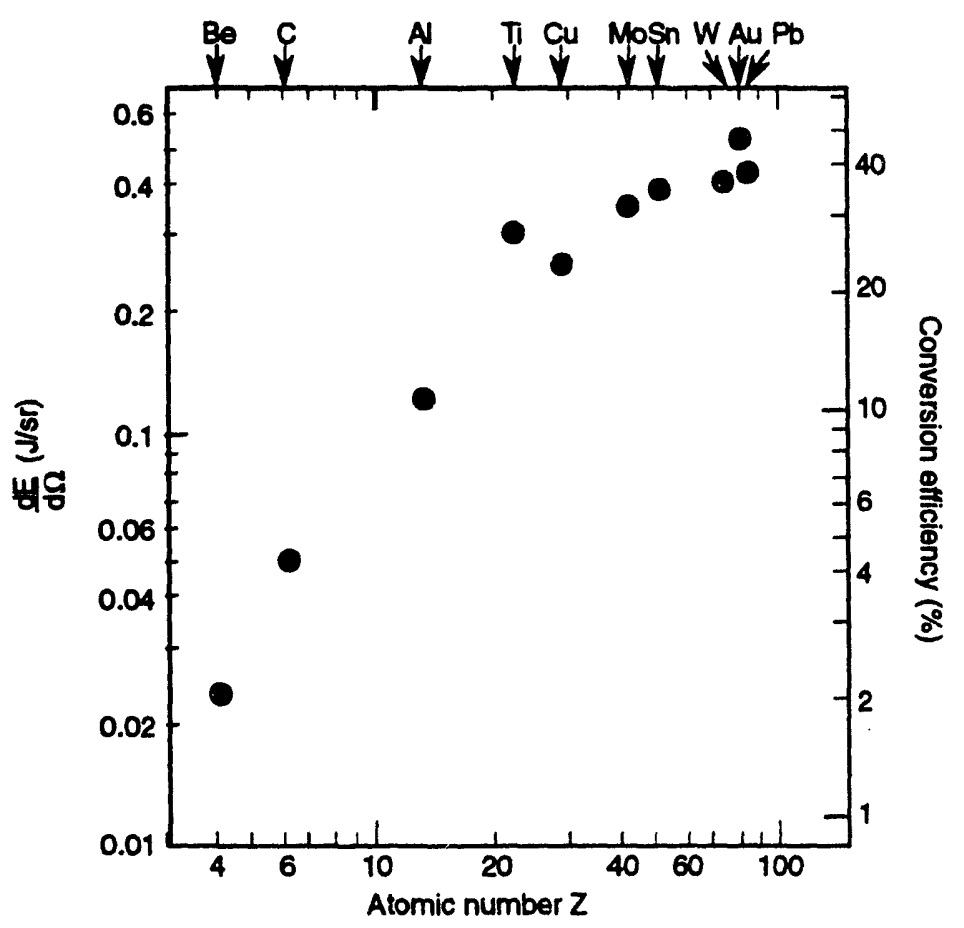

III.H.1.d-3) Absolute conversion efficiency of laser-irradiated target of different elements from Be through $\mathbf{P b}$.

\section{e) Angle of Incidence vs Conversion Efficiency}

The next pertinent issue concerns the effect of the angle of incidence of the laser with respect to the target surface normal on conversion efficiency. The reason the angular dependence is important is that to irradiate a sample with the $x$-rays from the laserconversion target there are two obvious geometries. First, one places the sample at the rear of a high- $Z$ foil that is thin enough to let $x$-rays through, but thick enough to stop laser light from being transmitted (see Figs. III.H.1.a-1 and -2 for information). Second, if one were trying to enhance the radiation, since the front surface $x$-ray conversions are higher, an angled arrangement is necessary.

In Fig. III.H.1.e-1 we show two quantities as a function of angle of incidence of the laser. ${ }^{40}$ First, on the left-hand axis is the absorption of the laser light by the target. This is represented by the open circles. The right-hand axis is the $x$-ray conversion efficiency measured relative to the absorbed energy. To convert this to the conversion efficiency relative to the incident energy, multiply the $E_{x} / E_{a b s}$ by the absorption percent. This gives, for example, for the $20^{\circ}$ result, a conversion efficiency of $46 \%$, which is in keeping with the $40 \%$ figure noted above. The efficiency does not drop off until the angle of incidence becomes greater than $45^{\circ}$. This alleviates experimental design constraints. The actual change in spectral shape as the angle is increased can also be shown not to deviate for angles less than $45^{\circ}$. These facts indicate the uniformity of the spectrum at angles less than $45^{\circ}$ and gives an indication that angles up to as high as $50^{\circ}$ preserve a similar $x$-ray spectral character. 


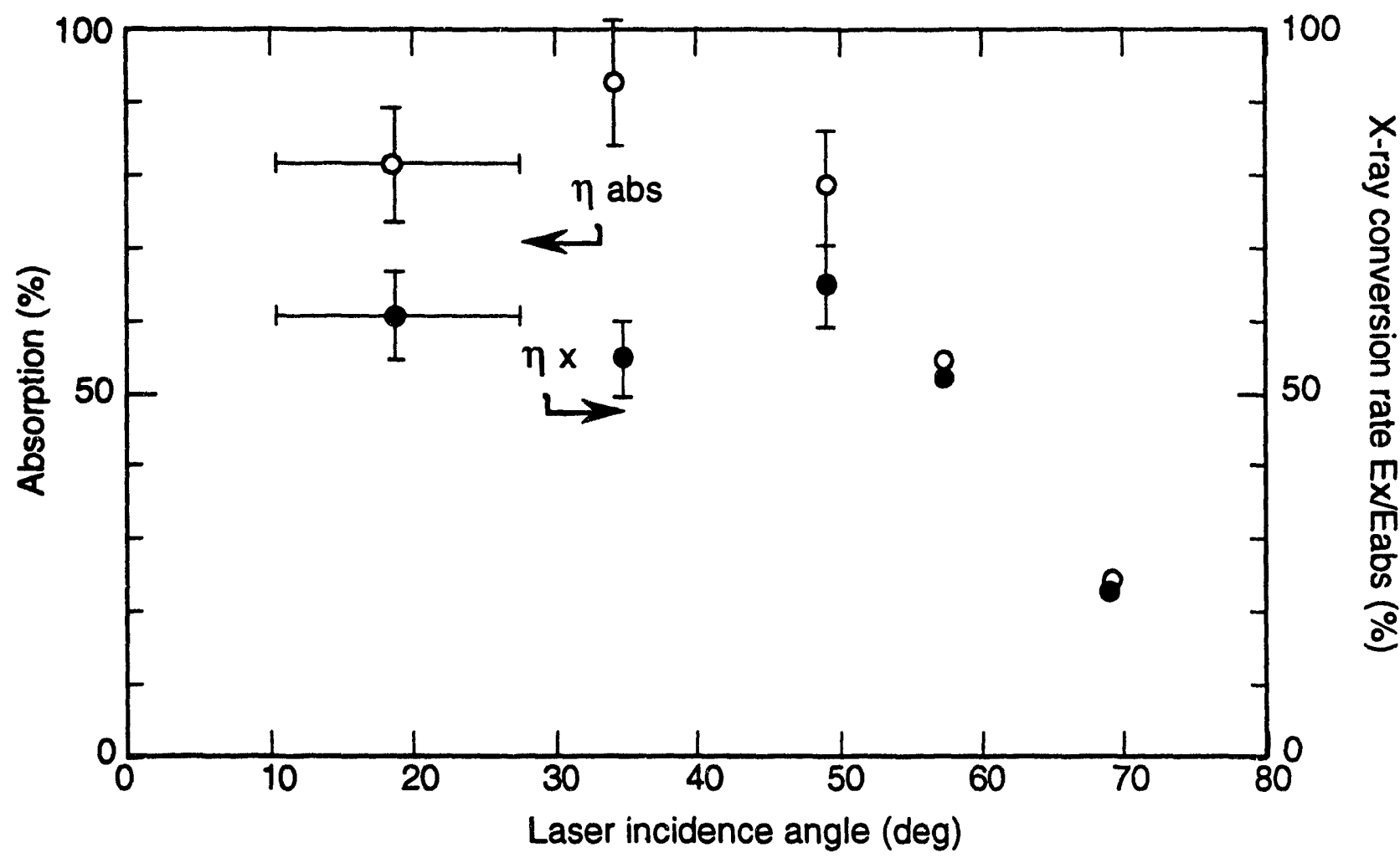

III.H.1.e-1) The effect of angle of incidence of the laser on $x$-ray conversion efriciency for intensity of $10^{14} \mathrm{~W} / \mathrm{cm}^{2}$ on a solid Au target. The left hand axis refers to the open circles which measure the absorption of the laser light. The right hand axis refers to the filled circles and represents the $x$-ray conversion efficiency relative to the absorbed laser energy.

\section{f) Laser Intensity vs Conversion Efficiency}

The next important question concerns the conversion efficiency from the laser light to $x$-rays as a function of intensity on target. In Fig. III.H.1.a-1 we saw that the conversion efficiency is roughly constant from $10^{12}$ to $10^{13} \mathrm{~W} / \mathrm{cm}^{2}$. However, the question arises: What is the behavior at higher or lower irradiance? There is data from the rear side of the target to indicate that the conversion stays roughly constant, at least to $10^{14}$ $\mathrm{W} / \mathrm{cm}^{2}$. Beyond this irradiance the onset of laser-driven parametric processes will start to play a role and the benign nature of the $x$-ray conversion process is not guaranteed. Note that with the advent of smoothed beams this statement should be seen as worthy of investigation.

On the lower irradiance side, the broad definition of the soft $x$-ray region, from $50 \mathrm{eV}$ through $1250 \mathrm{eV}$, allows a wide range of temperatures over which the plasma will emit $\mathrm{x}$-rays in this spectral band. Clearly the source will peak at lower energies as the laser intensity drops and the total energy in the $\mathrm{x}$-ray band will drop. However, 
although we have no data on the conversion efficiency below $1012 \mathrm{~W} / \mathrm{cm}^{2}$, a reasonable estimate is to assume that:

- The absorption stays high at irradiances above $109 \mathrm{~W} / \mathrm{cm}^{2}$, the threshold for plasma formation.

- The absorbed laser light will radiate as a blackbody radiator.

- Therefore, we equate the laser intensity to the power radiated, i.e., the StefanBoltzmann relationship: $\mathrm{T}_{\mathrm{ev}} \sim\left(10^{-5} \mathrm{I}_{\text {laser }}\right)^{0.25}$

Here $T_{e v}$ is the plasma temperature in $\mathrm{eV}$ and $\mathrm{I}_{\text {laser }}$ is the laser intensity in $\mathrm{W} / \mathrm{cm}^{2}$. Thus, for an intensity of $10^{12} \mathrm{~W} / \mathrm{cm}^{2}$ the temperature of the plasma will be $\sim 18 \mathrm{eV}$, indicating that the peak of the emission-were it a blackbody emitter-would be at $50 \mathrm{eV}$. The total energy emitted above $50 \mathrm{eV}$ would be half the laser energy. Since this is, in the spirit of the approximation, $40 \%$, one can extend this rough estimate of conversion efficiency down to intensities of $\sim 1010 \mathrm{~W} / \mathrm{cm}^{2}$. But, remember there is no data at these intensities yet.

\section{2) Spectral Narrow Sources}

The conversion of the laser radiation to discrete lines or narrow bands of lines represents a distinct field of study. The usual motivation for the study of conversion into narrow spectral lines has been the development of backlight (i.e., absorption) sources for use as a probe of the hot dense matter. Because this has been the motivation, there is also an interest in the spatial extent of the source. In a manner similar to that used to estimate the conversion efficiency of the quasi-continuous source, it can be broadly assumed that one can attain conversion efficiencies into single spectral line of $0.1 \%$ to $1 \%$, with the lower efficiency being for higher $\mathrm{x}$-ray energies than $4 \mathrm{keV}$, and the higher efficiencies being relevant for transition energies below $4 \mathrm{keV}$.

\section{a) Effect of $Z$ on Emission}

The effect of $Z$ on the emission is to change the character of the emission from single spectral lines to bands. As shown in Fig. III.H.1.b-2, the Au M band is an example of the type of spectral structure one can expect. The conversion efficiency into these band-like structures is approximately $5 \%$ for $0.53-\mu \mathrm{m}$ light.

In Fig. III.H.2.a-1 we show a compilation of the results of experiments on several elements. ${ }^{41}$ The experiments irradiated planar target of the material of interest with intensities of 1014 to $1015 \mathrm{~W} / \mathrm{cm}^{2}$. Although there are some differences in the performance of the targets under intensity variation, it is not significant in this broad outline of the results. Included in the figure are the results for $\mathrm{Al}, \mathrm{Si}, \mathrm{Ti}, \mathrm{Ni}$, and $\mathrm{Zn} \mathrm{K}$-shell emission, and since this spans $Z^{\prime}$ 's of $13,14,22,28$, and 30 respectively, we have a fairly good indicator of the levels of conversion. Further, the conversion efficiency into the $\mathrm{Au}$ $(Z=69) M$-bands is also presented. Note that to change the conversion unit of this figure into fractional conversion in output joules, multiply the left-hand value $\xi_{x}$ by the $\mathrm{x}$-ray line energy given on the bottom axis by $1.6 \times 10^{-16} \mathrm{~J} / \mathrm{keV}$. For example, the $\mathrm{Au}$ 


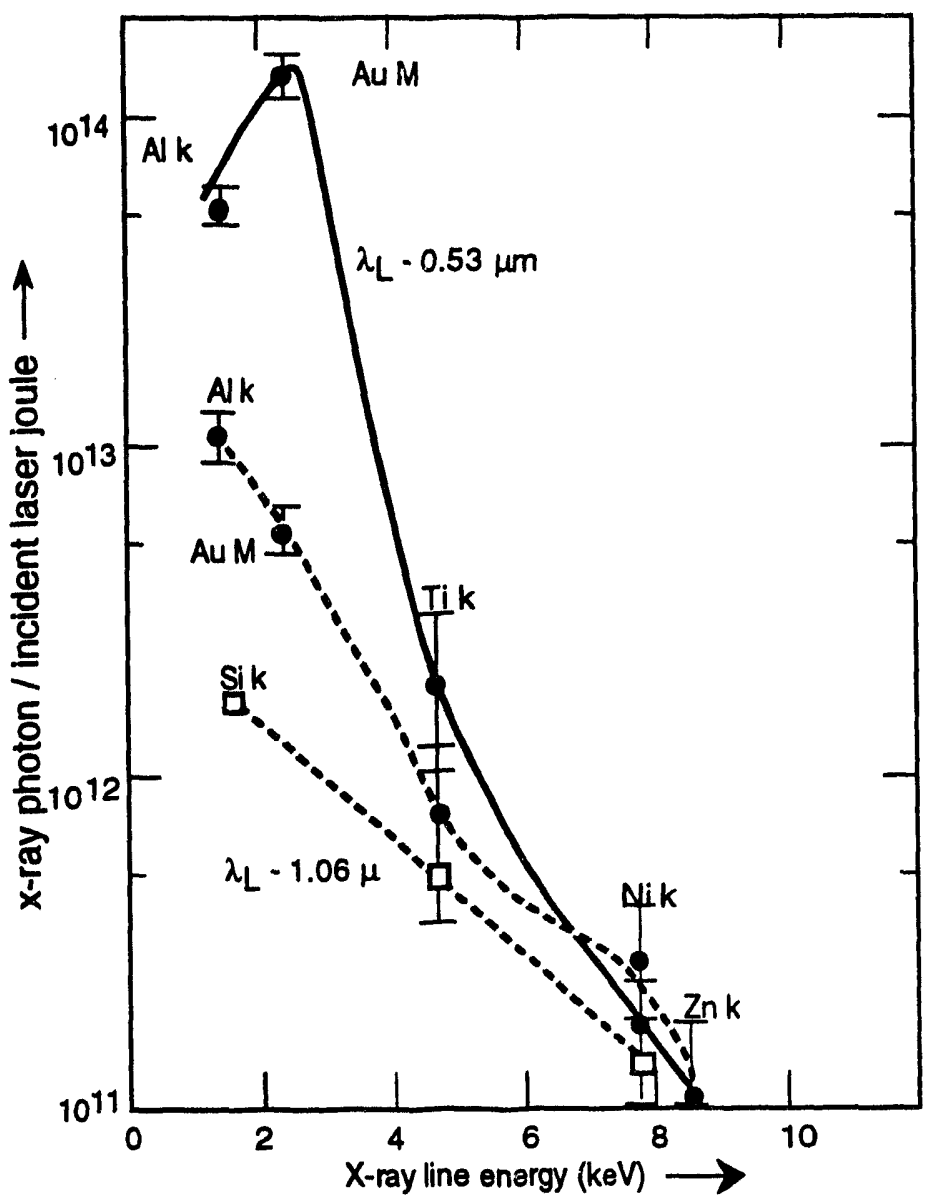

III.H.2.a-1) The conversion efficiency of various discrete transitions as a function of transition energy for intensities on the order of $\sim 10^{14} \mathrm{~W} / \mathrm{cm}^{2}$ on solid targets. The $\mathrm{K}$-shell lines of $\mathrm{Al}, \mathrm{Si}, \mathrm{Ti}, \mathrm{Ni}$, and $\mathrm{Zn}$ are shown as well as the $M$-band conversion of Au. The dashed lines tie together data for a 1.06- $\mu \mathrm{m}$ laser, and the solid line ties together the data for the $0.53-\mu \mathrm{m}$ laser.

$\mathrm{M}$ bands are at an energy of $\sim 2.5 \mathrm{keV}$ and the $\xi_{\mathrm{x}}$ is $\sim 1.3 \times 1014$, yielding $\sim 5 \%$ conversion. Further, in the figure the dashed line indicates the results with $1.03 \mu \mathrm{m}$ light, while the solid line connects those points with $0.53 \mu \mathrm{m}$ light.

\section{b) Comments on Sparseness of Data}

In the above we have given some simple rules for determining the conversion efficiency of laser light into $x$-rays. There are however, many gaps in the data, and particularly interesting is the lack of data from lower irradiances. For lower irradiances, that is, down to $1010 \mathrm{~W} / \mathrm{cm}^{2}$, the size of the source region can become large and the irradiation characteristics will be of interest. The levels of uniformity, angular dependence, temporal duration of the converted light, and spectral character are of interest. Although this data does not exist it is felt that one can use the simple rules above. Further work can, of course, be undertaken but this remains a very large field and it might be wise to have a first atteinpt at a design for the specific experiments of importance before seeking to obtain further quantitative data. 


\section{3) X-ray Laser Sources}

For two-dimensional plasma imaging, the spatial resolution is currently limited by the pulse length of the $x$-ray laser, or alternatively by the gate time of the detector. Gated detectors with large active areas have temporal resolutions, at best, in the range of 100 to $200 \mathrm{ps}$. For characteristic expansion velocities of $10^{7} \mathrm{~cm} / \mathrm{s}$ this corresponds to a spatial resolution of $10-20 \mu \mathrm{m}$, which is well below that possible with available imaging optics. Therefore, there exists a real need to produce $x$-ray lasers with pulse durations shorter than the 200-ps FWHM routinely produced with exploding foil targets.

Demonstrated $x$-ray lasers are either collisionally pumped systems in which inversions are produced in steady-state conditions, or recombination $x$-ray lasers, which rely on transient inversion to achieve gain. The gain duration in conventional, or quasistatic, recombination $x$-ray lasers is determined by cooling and recombination rates, and typically results in pulse widths $>200 \mathrm{ps}$. This allows little control over the output pulse length without significantly reducing the total gain. Collisionally pumped $x$-ray lasers have achieved large gain-length products and have been shown to operate over a wide range of pump conditions and a variety of targets. In addition, the wide-wavelength range over which collisionally pumped systems operate will allow one to take advantage, or alternatively avoid, line absorption in plasma-probing experiments.

To produce saturated $x$-ray lasers with pulse duration shorter than $20 \mathrm{ps}$, yielding an imaging resolutions of $\sim 2 \mu \mathrm{m}$, experiments which irradiate an exploding foil with multiple pulses have been performed. The concept is illustrated in Fig. III.H.3-1. The first pulse heats and explodes a thin foil target to produce a plasma with low density gradients. The second pulse then ionizes the preformed plasma to produce conditions suitable for high gain. The energy of the first pulse can be significantly lower than that of the second pulse since high electron temperatures are not necessary. This improves the efficiency of this approach by reducing radiation and thermal losses during the hydrodynamic expansion.

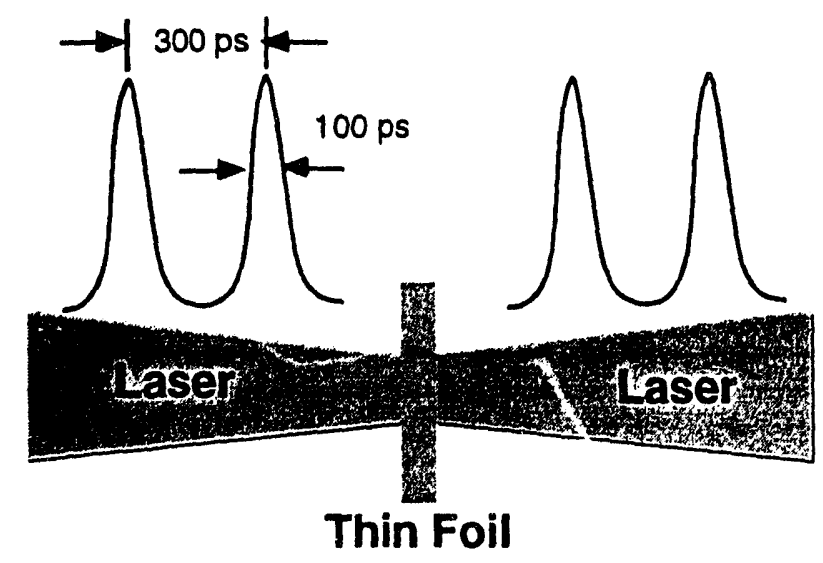

III.H.3-1) Schematic drawing illustrating the double-pulse irradiation technique for producing short-pulse x-ray lasers. 
To test this technique and illustrate the importance of density gradients in x-ray laser performance, experiments have been performed with yttrium $x$-ray laser targets. A key modification made to the laser beams was to introduce a tilt into the phase front of the beam. This allowed us to pump the foil in a traveling wave configuration as shown in Fig. III.H.3-2. This is important given the short gain duration and finite propagation time of the $x$-ray laser across the foil (i.e., $\sim 100 \mathrm{ps}$ for $3 \mathrm{~cm}$ foil).

In the experiment, a 3-cm-long foil consisting of $2000 \AA$ of yttrium on $1000 \AA$ of lexan was irradiated from both sides with two 100-ps pulses of $0.53 \mu \mathrm{m}$ laser light separated by $300 \mathrm{ps}$ and using traveling wave pump. The total intensity on target was $2.4 \times 10^{14} \mathrm{~W} / \mathrm{cm}^{2}$ and $1.6 \times 10^{14} \mathrm{~W} / \mathrm{cm}^{2}$ for the first and second pulses respectively. In Fig. III.H.3-3 the measured soft $x$-ray spectrum shows bright $J=2-1,155-\AA$ emission in 3 rd and 4th order of the grating. The effects of traveling wave pumping are clearly evident; the output is a factor of $\sim 200$ higher when the wave is traveling towards rather than away from the spectrometer.

a)
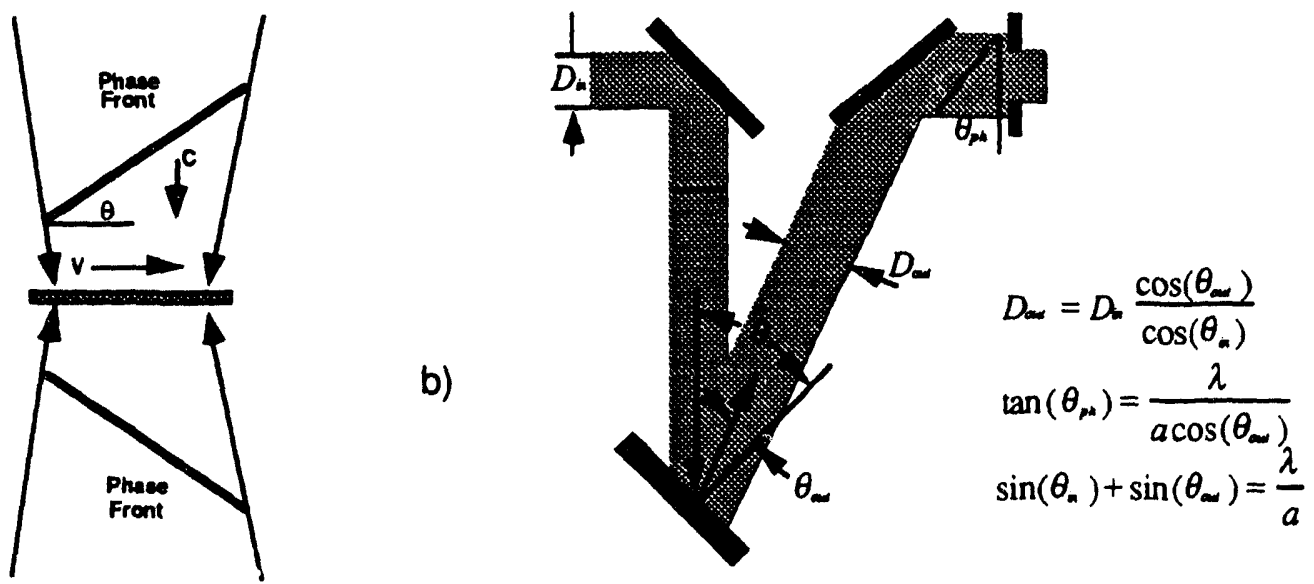

III.H.3-2) The traveling wave $x$-ray laser schematic. a) How optical beam is tilted to generate traveling wave pump along exploding foil. b) Grating technique used to tilt phase front to necessary angle.

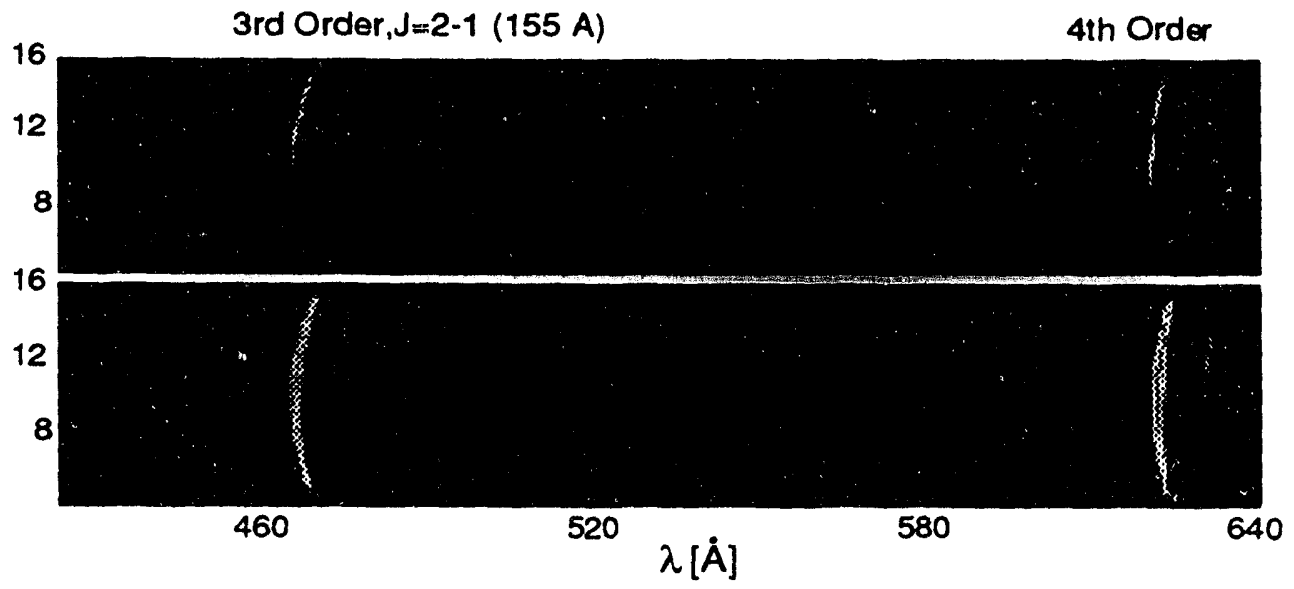

III.H.3-3) X-ray line emission in 3rd and 4th order with travel. ing wave. Top shows the case where the beam is going away from detector and bottom shows where beam is going toward detector. 
In Fig. III.H.3-4 we show the time-resolved emission spectra in both hard $x$-ray and XUV range. The hard $x$-ray emission (in Fig. II.H.3-4a) shows characteristic neon-like and fluorine-like 3-2 and 4-2 transitions which indicate that appropriate ionization conditions for gain are produced during both pulses. In contrast, the XUV spectra (in Fig. III.H.3-4b shows strong J = 2-1 x-ray laser emission at $155 \AA$ during the second pulse only. The most likely reason for this is that during the first pulse the high density gradients refract the $x$-ray laser emission out of the gain region. Figure III.H.3-5 shows the temporal evolution of both the $x$-ray laser and hard $x$-ray emission. The $x$-ray laser emission has a time duration of 45 ps FWHM, making this the shortest x-ray laser pulse width demonstrated.
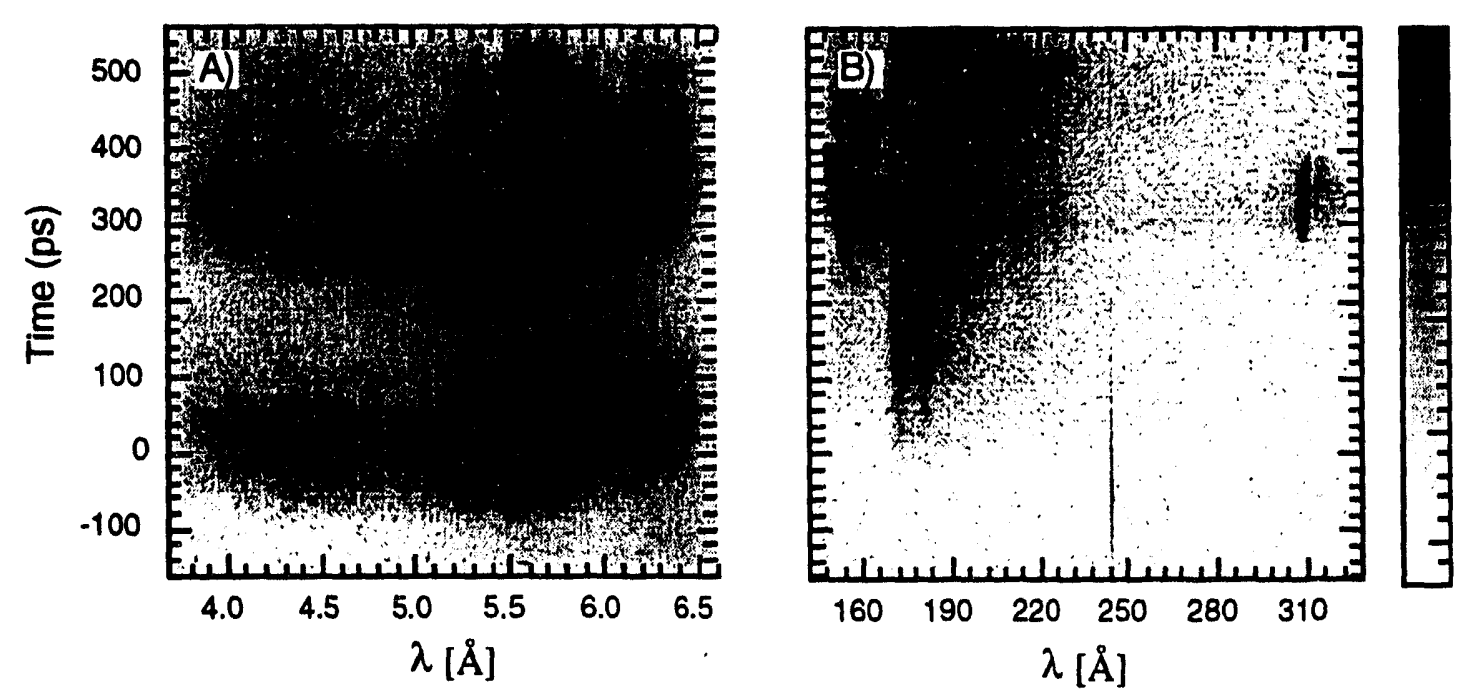

III.H.3-4) Time history of the emission from hard $x$-ray a) and XUV b) spectrum in neon-like yttrium. The strong $J=2-1$ transition at $155 \AA$ is clearly observed in first and second order.

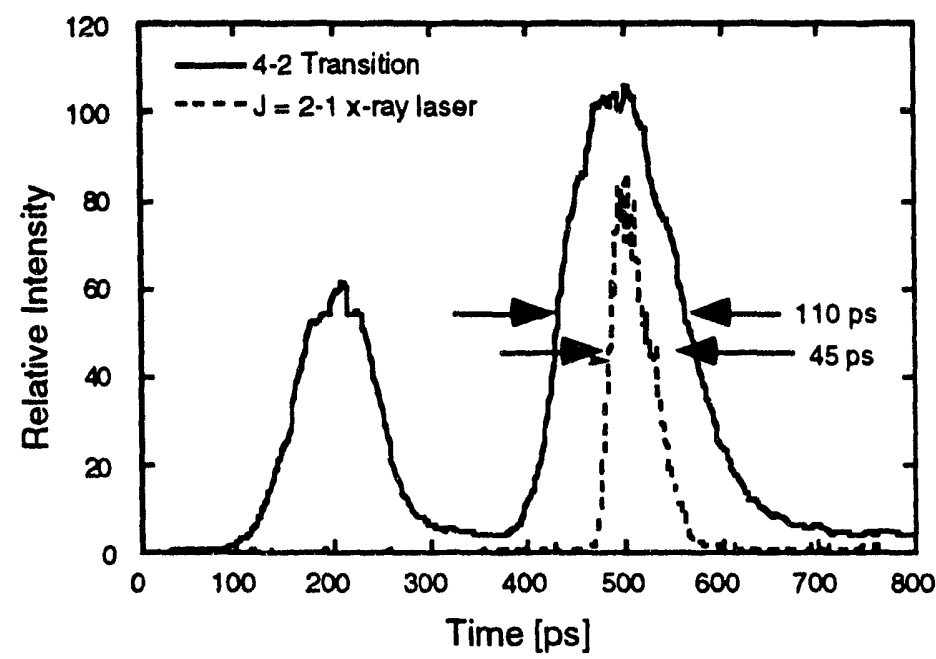

III.H.3-5) Time history of hard $x-r a y ~(4-2$ transitions) and $J=2-1$ x-ray laser transition in neon-like yttrium. 
Although in this experiment comparable intensities were used for both pulses, it would be clearly more efficient to reduce the intensity of the first pulse and increase the pulse separation to allow for expansion. Alternatively, a better pulse shape may consist of a low-intensity pedestal that irradiates the foil until the high-intensity short-pulse irradiation. Using this approach we should be able achieve saturation $x$-ray laser output intensities of $4 \times 10^{11} \mathrm{~W} / \mathrm{cm}^{2}$ with factors of $\sim 4$ less energy. Extending this technique to shorter output pulses will require shorter optical laser irradiation or a reduction in irradiation intensity to shorten the duration of neon-like ions.

\section{I) Plasma Physics}

The areas of plasma physics that have been addressed on high-energy lasers arise from two independent avenues. First, there are studies generated by the phenomena that are created by the laser interacting with a plasma. In this area one would have the instabilities enhanced by the laser plasma coupling, e.g., stimulated Brillouin scattering, stimulated Raman scattering etc. In the second are there are attempts to use the laser to emulate other phenomena occurring in nature. Here the study of interpenetrating plasmas and plasma flow in a magnetic field are examples to be presented.

\section{1) Interpenetrating Plasmas}

Plasma interpenetration occurs in beam-plasma systems, laser ablation experiments and in the context of geophysics and astrophysics. Therefore, interpenetration of counter-propagating plasmas can be considered a problem of fundamental interest in plasma physics. Single-fluid models are fundamentally unsuited because such codes enforce stagnation, resulting in unphysical temperatures and shock formation. Although methods for the collisionless regime are well developed, the intermediate regime is more difficult to model.

Recently, new computational methods have been investigated and applied to the simulation of colliding plasmas formed by laser ablation of parallel discs or foils. One technique is to follow multiple fluids that are coupled through the self-consistent electromagnetic fields as well as through collisional interaction. A different approach is to augment kinetic particle-in-cell calculations with algorithms for collisions. A typical result from this latter method is presented in the Fig. III.I.1-1, which shows a calculation of two laser-heated parallel plastic foils. The figure shows the ion density for each foil, clearly illustrating the interpenetration; the particle phase space shows the slowing down and heating in the center as the two plasma flows gently stagnate.

In Fig. III.I.1-2, an experiment performed on two solid targets is shown. The two laser beams impinge on the solid targets and create plasmas that expand toward each other. The targets are of aluminum and silicon to permit spectroscopic identification of the plasma positions. The measurements are made with a four-frame gated MCP coupled to a filtered pinhole. Thus, the images represent a two-dimensional map of the emission. 

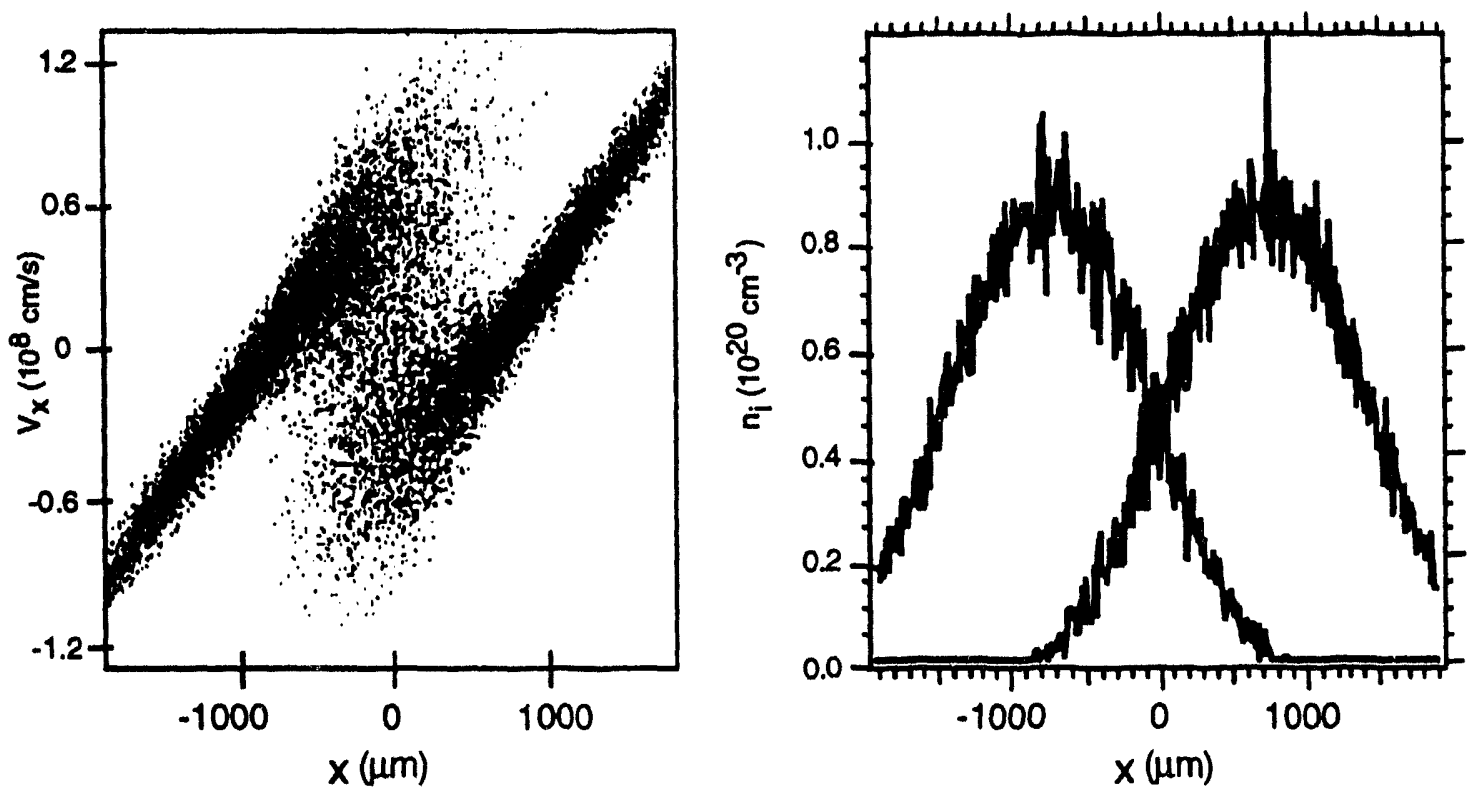

III.1.1-1) The simulation of an interpenetrating plasma showing the case for two exploding carbon foils, originally $3.2 \mu \mathrm{m}$ thick, separated by $1500 \mu \mathrm{m}$. a) The particle space result showing the ion density vs position measured from the center. The interpenetration is clearly viewed. b) The phase space view showing the stopping of the interpenetration due to collisions. Both are at $800 \mathrm{ps}$ after initiation.
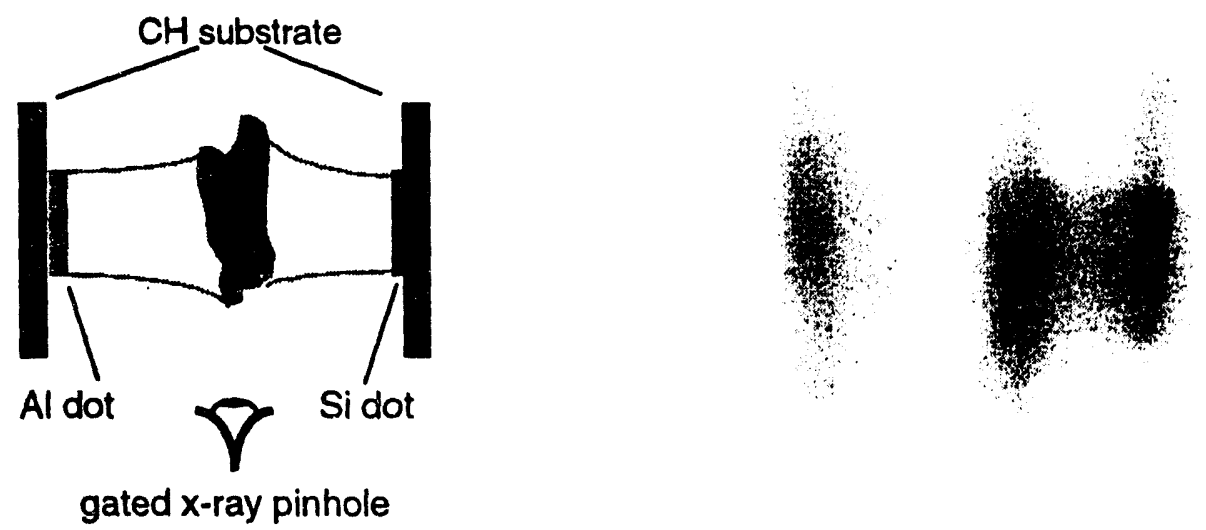
a) schematic
b) $x$-ray pinhole image

III.I.1-2) A schematic and an x-ray image of a colliding plasma experiment using two opposing beams to irradiate two facing solid targets. The Si dot is on the left and the Al dot is on the right. The dots are $250 \mu \mathrm{m}$ radius, the laser spot is $500 \mu \mathrm{m}$ radius, and the distance between the dots is $800 \mu \mathrm{m}$. The image on the right is taken with a filtered pinhole. 


\section{2) Plasma Streaming in Magnetic Fields}

The propagation and stability of collimated streams or jets of plasmas in magnetic fields is central to a number of important physical problems, including beam heating of magnetically confined thermonuclear plasmas, $\mathbf{4 2}$ the interaction of the solar wind with planetary magnetospheres, ${ }^{43}$ and the formation and equilibrium properties of extragalactic jets. ${ }^{44}$

A high-energy laser experiment conducted at total beam energies below $0.3 \mathrm{~kJ}$ has demonstrated the feasibility of studying high-energy jet phenomena with laser-produced plasmas. ${ }^{45}$ A cylindrical glass hohlraum, $3000 \mu \mathrm{m}$ long by $1000 \mu \mathrm{m}$ diameter, was irradiated with one or two beams of a laser operating at $1.06 \mu \mathrm{m}$ wavelength with a pulse length of $2 \mathrm{~ns}$ and energies ranging from 0.03 to $0.3 \mathrm{~kJ}$. The hohlraums were located at the center of a $24-\mathrm{cm}$ diameter Helmholtz coil which provided a spatially uniform and temporally constant magnetic field up to $10 \mathrm{kG}$ over regions and times of interest. The hohlraum axis was oriented perpendicular to the applied magnetic field.

The laser-produced plasmas from the inside of the cylindrical glass hohlraums were observed to evolve into plasma structures that were strongly collimated in the direction transverse to the hohlraum axis and the magnetic field but jetlike in the direction of the hohlraum axis. These jets were observed to propagate across the magnetic field lines at their initial velocities via the mechanism of an $\mathrm{E} \times \mathrm{B}$ drift. Shear in the velocity field of the jet led to the evolution of a classic Kelvin-Helmholtz-like instability on the edges of the plasma.

Figure III.I.2-1 shows an image taken of one such expansion into a 10-kG magnetic field from a hohlraum irradiated with a single beam at $0.1 \mathrm{~kJ}$. The image was obtained using an ultra-fast optical framing camera with 2-ns time resolution. In this experiment, the hohlraum end opposite to the laser-entrance hole was closed using an epoxy plug. At $850 \mathrm{~ns}$ after the laser was incident on the target, the maximum plasma jet velocity is $\approx 8 \mathrm{~cm} / \mu \mathrm{s}$ and a dramatic vortex structure has been produced. Both the jet formation and the observed instability may be relevant to a range of astrophysical phenomena.

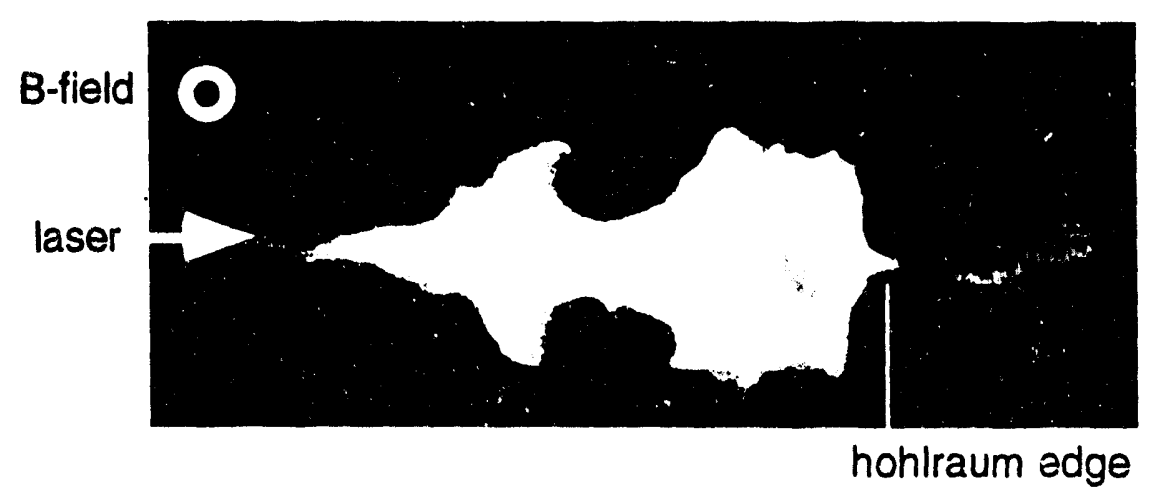

III.1.2-1) An optical image of a plasma streaming into a magnetic field. The plasma is emerging from a hohlraum on the right into a B-field that is coming out of the plane of the figure. The image is taken at $850 \mathrm{~ns}$ after the laser pulse started. Note the vortex structure. 


\section{3) Laser-Plasma Instabilities}

The coupling of high-intensity laser light to plasmas has been the subject of experimental investigations for many years. ${ }^{46}$ These experiments have focused on measuring a broad range of phenomena such as resonance and collisional absorption, filamentation, density profile and particle distribution modification, and the growth and saturation of various parametric instabilities. These phenomena depend on both the properties of the laser (e.g., intensity, wavelength, pulse length coherence) and the composition of the plasma.

Experimental studies of laser-plasma instabilities have become particularly important in recent years as a result of the vigorous research effort in laser-driven ICF. To some degree the success of ICF depends partly on mitigating the undesirable effects of two particular parametric instabilities. These instabilities are the stimulated Raman scattering (SRS) instability and the stimulated Brillouin scattering (SBS) instability.

The reasons these are of particular importance can be understood as follows. Electron Landau damping from the SRS instability produces fast electrons that can preheat the core of an imploding sphere prior to the arrival of the compression shock front. 47 The SBS instability can scatter a substantial fraction of the incident laser light, causing an overall reduction in the laser-to- $x$-ray drive efficiency and modifying the $x$-ray drive symmetry. Both of these instabilities degrade the target compression efficiency in a spherical implosion experiment.

In addition to its importance for ICF, high-intensity laser-plasma coupling presents an extraordinarily rich topic in the study of high-energy-density physics. For example, laser-produced plasmas provide a unique environment for the study of collisional and resoriance absorption of laser light. Numerous experiments using various kinds of target material and widely varying laser parameters have verified the general features of collisional absorption, such as the dependence of absorption on plasma temperature, scale length, laser wavelength and intensity. 48,49 Experiments investigating resonance absorption, which occurs at the critical density surface, show an expected dependence on the angle of incidence and polarization, $, 0,51$ but show some discrepancies in the absorbed energy that may be attributed to rippling of the critical density $\left(N_{c r}\right)$ surface. Density profile modification has been observed in experiments where resonance absorption is the dominant coupling mechanism. This profile modification can lead to harmonic generation in the back-reflected laser light. ${ }^{52}$

Laser-produced plasmas also provide a unique environment for the study of parametric instabilities. These instabilities can most simply be described as the resonant coupling of the incident laser light into two other plasma waves. Experi . rents on the stimulated Raman instability (SRS) mentioned above in the context of ICI, iv were an incident photon decays into an electron plasma wave and a scattered photon, have shown some important trends such as the generation of hot electrons from SRS and the dependence of the instability on plasma scalelength, laser intensity, and electron collisionality. 
Figure III.1.3-1 shows an example of the tirne-resolved SRS spectrum arising from a $\mathrm{CH} 2$ exploding-foil target. The foil was irradiated with a temporally square $1.0-\mathrm{ns}$ pulse of $0.35 \mu \mathrm{m}$ light with an intensity of $\sim 3 \times 10^{15} \mathrm{~W} / \mathrm{cm}^{2}$. The laser burns through the target during the pulse, producing an approximately parabolic density profile. This is seen in the figure, where the maximum density at which the SRS occurs drops from $\sim 0.22$ to $0.09 \mathrm{~N}_{\mathrm{cr}}$ as the foil explodes. Further, the time dependence of the short-wavelength cutoff implies a heating and then a cooling of the electrons as the foil expands. However, the details of this instability, such as the frequency spectrum, angular distribution of the scattered light, saturation mechanisms, threshold intensities, and starting noise level for the instability, are not well understood.

The two-plasmon decay instability occurs when the incident laser light decays into two electron-plasma waves. Experiments utilizing Thomson scattering have shown that the wavenumber for maximum growth is the same as predicted by linear theory. 53 These same experiments showed large levels of (short-wavelength) ion fluctuations that correlated with the two-plasmon decay Langmuir waves. Meanwhile, experiments have examined the saturation and long-time-scale evolution of this instability. 54

The stimulated Brillouin scattering instability, as mentioned above in the context of ICF, occurs when the incident laser decays into an ion acoustic wave and a scattered light wave. A number of interaction experiments have produced substantial backscattered and sidescattered light that is attributed to the SBS instability. ${ }^{49,55}$ The intensity of the scattered light seems to decrease as the wavelength of the interaction light decreases.

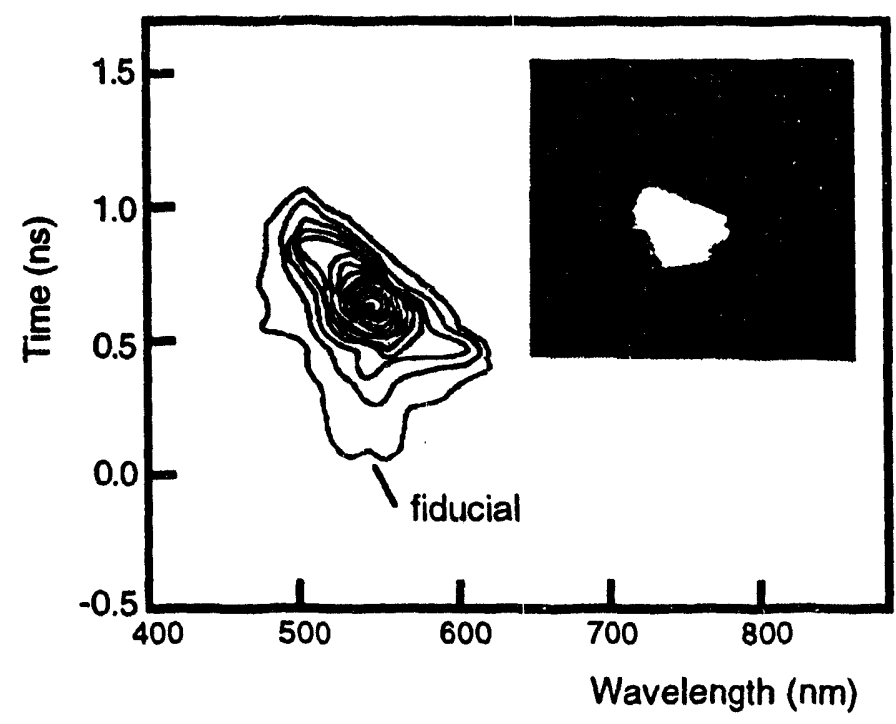

III.I.3-1) A contour plot of the intensity of the Raman-scattered light vs wavelength and time. The data image from a streak camera couplled to a visible spectrometer is shown in the insert. The experiment has a thin $\mathrm{CH}$ foil irradiated by a $0.35-\mu \mathrm{m}$ laser at $10^{15} \mathrm{~W} / \mathrm{cm}^{2}$. Note the timing fiducial used to indicate time relative to the incident laser pulse. 
The temporal growth rate, spatial behavior, and saturation characteristics of SBS were investigated using Thomson scattering and measurements of the backreflected light. ${ }^{56}$ The exponential growth in time was found to be in good agreement with linear theory. Experiments investigating the nonlinear effects of SBS have shown ion acoustic wave harmonic generation and a rapidly varying $100 \%$ modulation of the ion wave. 57

Some typical spectra of back-reflected light for a glass microsphere and a $\mathrm{CH}$ disk irradiated with 1.06- $\mu \mathrm{m}$ light are shown in Fig. III.I.3-2. Here the spectrum is blueshifted for the low- $\mathrm{ZCH}$ target shown, while the spectrum is red-shifted for the higher$Z$ target. Measurements of the spectral shift with different angles of incidence have been used to estimate the sound speed and plasma expansion velocity. Note that experiments using spatial and temporal laser-beam smoothing techniques, mentioned in the description of the laser facility, have shown a significant reduction in the level of SBS and SRS backscattered emission. ${ }^{58}$

Various parametric instabilities may occur at the same location in a plasma and as a result can interact with each other. One experiment investigating the interaction of SRS and SBS showed a strong correlation between the quenching of SRS plasma waves and the initiation of SBS ion waves. ${ }^{59}$ Another experiment showed that the spectrum of backscattered light from SRS depended on the presence of SBS. ${ }^{60}$ In these experiments the data showed that the temporal evolution of the "Raman gap," which is an observationally missing SRS signal that would be associated with regions of electron density from $\sim 0.18$ to $0.25 \mathrm{~N}_{\mathrm{cr}}$, could be explained in terms of the SBS scattered light. Although these experiments do not completely explain the gap, these experimental results are consistent with the assumption that an interaction exists between the SRS and SBS instabilities.

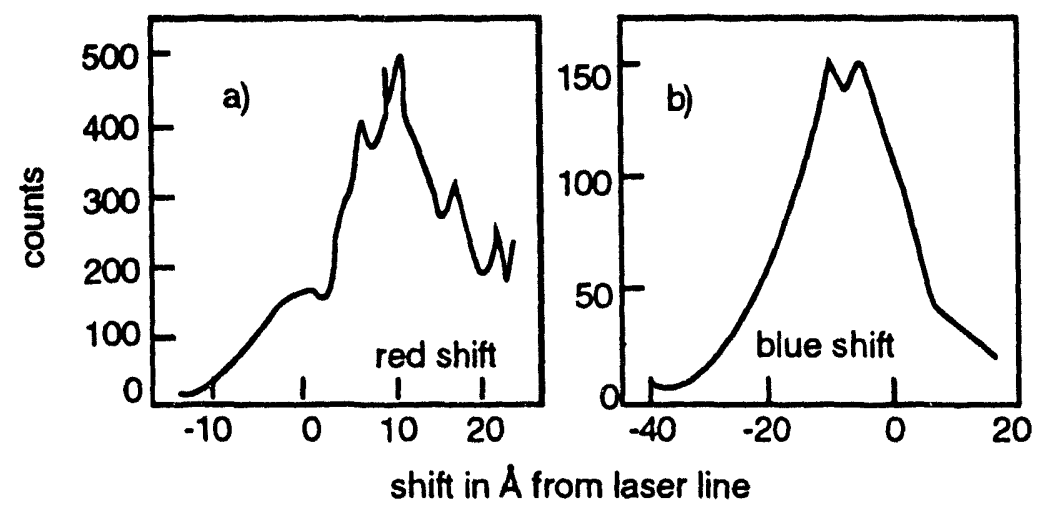

III.I.3-2) Spectra for the back-reflected light due to SBS. In a) the material is a glass microsphere, while in b) it is a parylene (C), disk. Note that the dominant shift changes signs for the two materials. 
The filamentation instability occurs when a small hot spot in the laser-intensity profile undergoes self-focusing as a result of thermal, ponderomotive, and/or relativistic effects. The result is that electrons, and eventually ions, are expelled from the filament, lowering the plasma density and causing the laser light to focus more tightly. This creates an unstable feedback loop where a tighter focus leads to higher intensity and more density depletion. Eventually, the instability saturates through diffraction effects, thermal absorption, or laser-light scattering by SBS and SRS instabilities.

Filamentary structures have been inferred from density perturbations measured using interferometry, shadowgraphy and dark-field imaging, and by imaging the second and three-halves harmonic emission.61,62 Other experiments designed to study the growth of a well-defined intensity perturbation show an increase in the spatial density perturbation that is consistent with ponderomotive filamentation. 63

An example of filamentation results is shown in Fig. III.I.3-3 for an experiment using a long scale-length plasma. The plasma was formed by focusing a 4-ns pulse of $1.05-\mu \mathrm{m}$ light onto a $\mathrm{CH}$ foil. The spot size was $\sim 1 \mathrm{~mm}$ and peak intensity was $\sim 6 \times 10^{12} \mathrm{~W} / \mathrm{cm}^{2}$. The plasma was then irradiated with a second, more intense, 300-ps pulse, with $\sim 8 \times 1014 \mathrm{~W} / \mathrm{cm}^{2}$. In Fig. III.I.3-3 the pattern of the second-harmonic emission is observed. Note the filamentary structures that extend up to $\sim 1 \mathrm{~mm}$ from the surface of the target, where the density should be on the order of $0.05 \mathrm{~N}_{\mathrm{cr}}$.

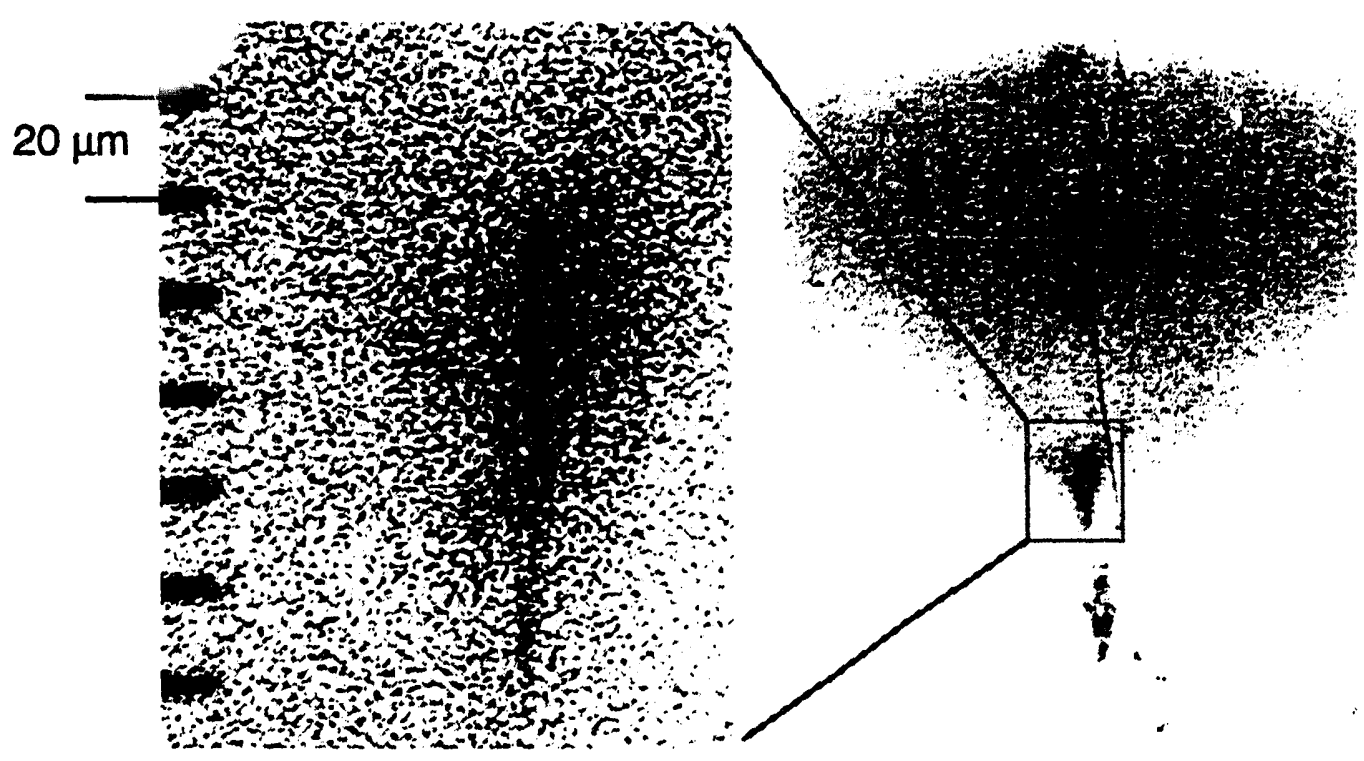

III.1.3-3) An image of laser-induced filamentation. The second-harmonic emission is observed from a preformed plasma irradiated with an intense $1.05-\mu \mathrm{m}$ laser. The image on the left shows the target surface in the entire spatial record. The right image with the scale shows a magnified section of one of the filaments. 
Notwithstanding the many results just mentioned, there remains a lack of detailed experimental knowledge of many aspects of these instabilities. For example, experiments investigating the two-plasmon decay instability at short wavelengths, sidescatter from SBS, and filamentation are ripe for investigation. Additional experiments studying the interaction of instabilities, forward Raman scattering, and the coupling of long duration shaped pulses of laser light are further areas of emerging importance.

\section{IV) Diagnostic Capabilities}

In contrast to some smaller facilities, the diagnostics on Nova are engineered to be routinely run on any shot by facility staff. This involved applying professional engineering standards to the diagnostics. The more usual notion of the standard operating procedure of several Ph.D.-level scientists working for many weeks to get their principal diagnostic installed for their series of experiments, and after an intense campaign of experiments removing their diagnostic, has been avoided.

In the long run, this is an efficient use of resources. Also it is necessary for efficient facility scheduling, as Nova runs in the mode of doing four to six different sets of experiments in any one week. This mode of scheduling precludes the exclusive use of a target chamber for blocks of a week or more, but has the advantage of giving experimentalists time to analyze a block of data before proceeding with an experimental series.

To achieve a high standard of engineering for Nova's diagnostics, experimentalists work with engineers and technicians for many months to design, build, and implement the diagnostics, with the goal of implementing diagnostics that can be routinely run by facility staff for any user.

Although it is $2.4 \mathrm{~m}$ in radius, the ten-beam target chamber will pump to vacuum in less than one hour. It is not routinely vented during the four- or five-day shooting week. For shots where the target geometry is unchanged, this allows shots to be fired without realignment of the laser beams. As a result, diagnostics are designed to be operated without venting the target chamber.

For flexibility, all of the $x$-ray imagers and $x$-ray spectrometers that operate close to the target have been made to be interchangeable into one of six standardized vacuumload lock and manipulator devices, known as SIMs (for six-inch manipulators). In excess of twenty diagnostics are now mounted on the carts that fit into the SIMs. This standardization allows key diagnostics to be moved from one location on the target chamber to another location over a period of about 1 hour, so that the configuration of target diagnostics can be rapidly changed for different experimental campaigns. This typically happens every 2-4 shots, the number of consecutive shots that are fired in any one campaign out of the 30 or so shots that are fired most weeks. 


\section{A) Overview of the Target Chamber Diagnostics}

Table IV.A.1 lists the dozen different $x$-ray imaging diagnostics available on Nova. The imagers are reflective grazing incidence $x$-ray microscopes, pinholes or coded apertures or point-projection systems. It is important to have time resolution, and so the detectors for the imagers are $\mathrm{x}$-ray streak cameras, gated microchannel plate (MCP) detectors, or simply x-ray film with flash backlighting.

Table IV.A.1 also shows the x-ray spectrometers available. These are crystal spectrometers (Bragg or Laue), broad-band diode arrays, or grazing incidence grating spectrometers. Again, the detectors are either $\mathrm{x}$-ray streak cameras, gated MCP detectors, or $x$-ray film.

The neutron diagnostics are yield (by activation), high- and low-resolution energy spectra, emission-time diagnostics, and neutron imaging.

The optical diagnostics are streaked optical spectrometers, used mainly for stimulated Brillouin and Raman scattering in either one location or in an array of different angular locations; optical and UV imagers that either go onto streak cameras or gated optical imagers; and arrays of calorimeter modules.

Finally note that in Table IV.A.1 we have listed the abbreviated name of the diagnostics. In many of the figures one will find these abbreviations used. The various uses of the diagnostics have been covered in the section above.

\section{B) X-ray Imagers}

Nova has had for several years reflective grazing incidence $\mathrm{x}$-ray microscopes, two of the Kirkpatrick-Baez design and one of the Wolther design. The detectors for one of the four-channel Kirkpatrick-Baez $x$-ray microscopes is film. The other microscope has four time-gated $x$-ray detectors using gated $\mathrm{MCP}$ (microchannel-plate) technology. 64

The Wolther microscope has approximately eight microns spatial resolution, although in one direction there is a large scatter caused by the surface roughness of the $x$-ray optic. This instrument has been widely used on Nova. ${ }^{65}$ The collecting solid angle of the optic is large enough that only a small sector of the annular imaging region need be used to form an image. Five separate $5^{\circ}$ sectors of the annular optic are used to make it into a five-channel system, with an $x$-ray streak camera on one channel and gated MCP detectors on each of the other four channels. ${ }^{66}$ This instrument has been widely used on $x$-ray backlighting experiments on Nova where accuracy, ease of alignment, and sensitivity are important. Note that the solid angle/channel is $3 \times 10^{-6} \mathrm{sr}$ compared to about $10^{-7} \mathrm{sr}$ for a Kirkpatrick-Baez microscope, but resolution and high-energy use, above $3 \mathrm{keV}$, are not crucial.

For higher $x$-ray energy imaging, simpler systems are also being used on Nova. Crucial information on symmetry and the mix of pusher material into a fuel is obtained 
Table IV.A.1. Diagnostics available on Nova, with their abbreviated names. These include $x$-ray imagers and spectrometers, neutron diagnostics, and optical imagers and spectrometers.

\begin{tabular}{|c|c|c|c|c|c|}
\hline \multirow{2}{*}{$\frac{X \text {-ray imagers }}{\text { Wolther } x \text {-ray microscope }}$} & \multicolumn{5}{|c|}{ Neutron diagnostics } \\
\hline & $22 X$ & 1 & Yield & $\mathrm{Cu}, \ln \operatorname{scin}$ & 3 \\
\hline Gated x-ray pin-hole camera & $\begin{array}{l}\text { GXI, WAX, } \\
\text { GACS, GSIX }\end{array}$ & 4 & $\begin{array}{l}\text { Bang time } \\
\text { Burnwidth }\end{array}$ & $\begin{array}{l}\text { NETMCP, GaAs } \\
\text { NTD \& GaAs }\end{array}$ & $\begin{array}{l}3 \\
2\end{array}$ \\
\hline Gated soft $x$-ray framing camera & SXRFC & 1 & $\begin{array}{l}\text { High-resolution high sensitivity } \\
\text { spectrometer }\end{array}$ & & \\
\hline Streaked soft $x$-ray imager & NSDSS & 1 & $\begin{array}{l}\text { spectrometer } \\
\text { Medium-resolution }\end{array}$ & LaNSA & 1 \\
\hline Ring-aperture microscope & RAM & 1 & $\begin{array}{l}\text { Medium-resolution } \\
\text { neutron spectrometer }\end{array}$ & NTOF & 3 \\
\hline Streaked slitarray imager & SSC/SMP & 2 & Ultrahigh-resolution spectrometer & INTOF & 1 \\
\hline Kirkpatrick-Baez microscopes & $8 X$ & 2 & $\begin{array}{l}\text { High-resolution high-sensitivity } \\
\text { spectroscopy }\end{array}$ & LANL $\pi$ & 1 \\
\hline $\begin{array}{l}\text { Axial pin-hole cameras } \\
\text { Large-area backlighting }\end{array}$ & APH & & Neutron imager & NPAM & \\
\hline Point-projection spectroscopy & PPS & 2 & & & \\
\hline Soft x-ray microscope & & 1 & & & \\
\hline XRL beam divergence camera & Cube & 1 & & & \\
\hline XRL spatial-coherence diagnostic & & 1 & Optical spectrometers an & nd imagers & \\
\hline X-ray spectrometers & & & Streaked/gated imager & SOP & 2 \\
\hline Streaked crystal spectrometers & $\begin{array}{l}\text { NSCS, } \\
\text { Keanetech }\end{array}$ & 2 & $\begin{array}{l}\text { Streaked optical spectrometers } \\
\text { Multiple streaked spectroscopy }\end{array}$ & $\begin{array}{l}\text { SOS,BSS } \\
\text { MATRES }\end{array}$ & $\begin{array}{l}4 \\
1\end{array}$ \\
\hline $\begin{array}{l}\text { High-resolution } \\
\text { streaked spectrometer }\end{array}$ & HICKS & 1 & $\begin{array}{l}\text { Spatially discr. streaked } \\
\text { optical spectroscopy }\end{array}$ & SDOSS & 1 \\
\hline Static crystal spectrometers & Henway,POS & 6 & Calorimeter array & EBM & 1 \\
\hline $\begin{array}{l}\text { High-resolution } \\
\text { crystal spectrometer }\end{array}$ & HOPS & 1 & Full-beam backscatter & sos5 & 1 \\
\hline Gated crystal spectrometer & TOPS & 1 & & & \\
\hline Gated imaging XUV spectrometer & IXUVS & & & & \\
\hline Laue spectrometer & HETS & 1 & & & \\
\hline Low-resolution x-ray diode array & Dante & 2 & & & \\
\hline $\begin{array}{l}\text { Low-resolution high-energy } \\
\text { fluorescers }\end{array}$ & FFLEX & 2 & & & \\
\hline Spatial coherence diagnostic & & 1 & & & \\
\hline Grazing-incidence spectrometer & COFFIN & 1 & & & \\
\hline High-resolution spectrometer & HIRES & 1 & & & \\
\hline $\begin{array}{l}\text { Time-resolved soft x-ray } \\
\text { spectrometer }\end{array}$ & SFFD & 1 & & & \\
\hline $\begin{array}{l}\text { Gated grazing-incidence } \\
\text { spectrometer }\end{array}$ & MCPIGS & 1 & & & \\
\hline
\end{tabular}


from imaging implosion cores at higher photon energy and better spatial resolution. The higher energy implies high sensitivity, and the requirement of good spatial resolution implies good time resolution to freeze plasma motion.

To tackle this problem, gated pinhole and coded aperture systems have been used. To ensure adequate sensitivity, the $5-10-\mu \mathrm{m}$ pinholes of the instruments are brought as close as $5 \mathrm{~cm}$ from the target. As the instruments are used to record signals from $\geq 3 \mathrm{keV}$, relatively robust $\mathrm{x}$-ray filters can be used, allowing the pinholes to survive the target shrapnel from the $20-40 \mathrm{~kJ}$ on target for a few shots.

Time resolution is achieved by gating $0.5-\mathrm{mm}$-thick coated MCPs. Several coating configurations are presently routinely used, a four-channel open-ended strip configuration, a 16-channel serpentine arrangement, and 16 channels onto four parallel strips. The motional blurring is given by

$$
\delta \mathrm{x}=\mathrm{v} \delta \mathrm{t}
$$

and so the 80-ps gate time that is easily achieved is adequate for $10-\mu \mathrm{m}$ resolution with velocities of approximately $10^{7} \mathrm{~cm} / \mathrm{s}$. For improved resolution, 30-40-psec gate times are becoming available with thinner MCPs.

X-ray backlighting has been in use for laser-produced plasmas for several years, but for implosions and in-flight measurements of higher density planar foils, higher energy backlighting is required. Several developments have made higher energy $(\sim 5-7 \mathrm{keV})$ streaked $\mathrm{x}$-ray backlighting possible. The most important are:

- Random phase plates are now used on the backlighting beams to produce a smooth, virtually monochromatic source of $x$-rays by irradiating, for example, an iron disc at $\sim 1015 \mathrm{~W} / \mathrm{cm}^{2}$.

- A slit imager onto a close-in x-ray streak camera with the photo cathode about $50 \mathrm{~cm}$ from the plasma allows for adequate sensitivity for recording this backlighting source.

For soft $x$-ray studies, two gated and streaked soft $x$-ray imaging instruments are in use. A schematic of the gated soft $x$-ray framing camera is shown in Fig. III.G.1.b-1. For two soft channels, a combination of grazing incidence mirrors and filters defines two bands of soft $x$-rays. The third and highest energy channel has its pass band defined by a filter. Images in $\mathrm{x}$-ray bands at $500 \mathrm{eV}, 1 \mathrm{keV}$ and $2.5-3 \mathrm{keV}$ are recorded with 100 -ps gate time at four different times, giving twelve images in total. This instrument allows the spatial evolution of the soft $x$-ray emitting region of a laser plasma to be accurately measured.

A major diagnostic technique for obtaining spectral and spatial information simultaneously is point projection spectroscopy. ${ }^{67}$ In this technique one or two of the backlighting beams are focused onto one or two $15-20-\mu \mathrm{m}$ coated fibers, to produce a flash, broad-band of quasi-continuum $x$-rays, from a point source. An image is formed by the 
shadow of the object that this "point" projects after the x-rays have been reflected off a Bragg crystal. As reflection only occurs when Bragg's law is satisfied, the shadow has spectral resolution as well as spatial resolution.

The $x$-ray laser researchers are characterizing the coherence, both spatial and temporal, of $x$-ray lasers, and beginning to demonstrate biological applications. The spatial coherence of laboratory $x$-ray lasers has been measured using a diagnostic based on partially coherent diffraction from a uniformly redundant transmission slit array. ${ }^{68}$

\section{C) X-ray Spectrometers}

In the crystal regime, there are several static survey instruments, called Henways, that use convex crystals at a distance of about 1 meter from the plasma for survey work from $1.5 \mathrm{keV}$ to about $10 \mathrm{keV}$.

There are also streaked crystal spectrometers, ${ }^{69}$ which on the ten-beam target chamber use flat crystals on the front of a close-in $x$-ray streak camera at either 3 to 6 degrees Bragg angle, or at a much larger Bragg angle for high resolution. For implosion work there has been an emphasis on high-energy, high-resolution spectroscopy because of the need for the line emission to penetrate the compressed pushers. Argon dopants have been successfully used to measure density from line broadening, but for future implosions, higher energy $x$-ray emission with higher spectral resolution will be required. With a large Bragg angle and a PET crystal, resolving powers of about 2000 have been achieved in streak mode at photon energies of about $6 \mathrm{keV}$.

For accurate absolute measurements of broad-band spectra, an array of fifteen calibrated $x$-ray diodes, called Dante, is used with filters and grazing incidence mirrors to isolate channels between $100 \mathrm{eV}$ to $>1.5 \mathrm{keV} .70$ This was one of the core diagnostic systems of Nova, and although originally designed for 1-ns recording, the photocurrent is now commonly recorded with fast oscilloscopes (e.g., Tektronix SCD 5000), giving a time resolution FWHM of 135 ps with some small ringing at 300-400 ps. This allows the time-resolved measurements of the $x$-ray conversion efficiency to be routinely made.

There are several soft $x$-ray instruments enumerated in Table IV.A.1. A particularly high resolution grazing-incidence spectrometer, HIRES, can achieve a spectral resolution of 8,000-26,000 in the 100-200- $\AA$ range when used with an $x$-ray streak camera, and up to 35,000 when used with soft $x$-ray film. This instrument was recently used to measure the line width of the dominant laser transition in Ne-like Se $x$-ray laser plasmas and to observe the scaling of the line width with amplifier gain-length product. ${ }^{71}$

The Streaked Flat Field Spectrometer (SFFS) is a time-resolved XUV spectrometer that utilizes a Harada varied line space grating. ${ }^{72}$ The grating has a flat focal plane that lies almost perpendicular to the $x$-ray propagation direction, allowing the use of a transmission photocathode $x$-ray streak camera. The instrument is sensitive from about $400 \AA(30 \mathrm{eV})$ to $\sim 10 \AA(1000 \mathrm{eV})$. With a 1200-line-per-mm grating with high efficiency in higher orders, up to 6 th order has been seen at $44.83 \AA$, and the instrument is used to 
view short-wavelength $\mathrm{x}$-rays, such as $44.83-\AA \mathrm{x}$-ray laser emissions, in multiple orders, extending the dynamic range of the spectrometer and allowing a resolution of $\lambda / \Delta \lambda$ $\sim 500$ to be achieved in some measurements (e.g., $44.83-\AA$ in 5 th order).

For shorter wavelength spectra, the SFFS can be used with a 2400 -line-per-mm grating. The two gratings are both installed in the spectrometer and either can be selected by translating them into position. The wavelength range is determined by an Ircoated mirror, midway between the detector and the grating and almost parallel to the grating surface. The mirror relays the spectrum from the grating to the photocathode. Unlike similar instruments that rely on moving the detector to vary the wavelength coverage, the SFFS keeps the detector fixed and moves the relay mirror. This feature allows the spectrometer to be re-entrant, increasing sensitivity as the spectrometer is close to the targets.

Like all the streaked diagnostics on the two-beam target chamber (Keanetech, XCSS, XRL power meter, XRL coherence measurement, SOS-3, SOS-5, PCDs, Dante, HSI, High Flux, UVImager, High Res, 2-Beam Incident Streak, Laser Diagnostic Station), the SFFS has a fiber-optic timing fiducial that allows the recorded spectra to be referenced to the incoming irradiation and other diagnostics to $\sim 30$-ps precision. The timing fiducial also allows for the testing and timing of streak cameras without firing the entire laser chain.

The Micro-channel Plate Intensified Grazing Incidence Spectrograph (MCPIGS) is a grazing-incidence XUV spectrometer with a gated microchannel plate detector. The instrument is a Rowland circle geometry spectrometer with a $2^{\circ}$ angle of incidence on the $998.8 \mathrm{~mm}$ radius grating. The detector is a $150-\mathrm{mm}$-long curved microchannel plate that lies along the Rowland circle with several configurations of strips which are gated off at intervals using a Si Auston switch triggered by the fiducial laser beam. With seven gratings available, a broad range of XUV spectra can be surveyed with this instrument. There are two MCPIGS spectrometers on the Two-Beam chamber. One lies in the equatorial plane to view $x$-ray laser output emitted in a narrow beam perpendicular to the plane of the Nova foci. The other MCPIGS spectrometer views the plasma from an off-axis direction.

\section{D) Optical Diagnostics}

The optical and UV diagnostics are also listed in Table IV.A.1. One of the most ubiquitous diagnostics is the streaked optical imager, called the SOP (for streaked optical pyrometer). This instrument is an $\mathrm{f} / 10$ Cassegrain telescope with a magnification of 8.47, with a resolution that is close to diffraction-limited. The instrument is most commonly used to record shock breakout from witness plates, for which purpose the image is recorded onto a UV-sensitive streak camera. Alternatively, the image can now be recorded onto a gated optical imager.

There are several streaked optical spectrometers at fixed locations on the two target chambers (SOS1, SOS2, SOS3, SOS4 and SOS5). These are primarily used to measure the stimulated Brillouin and Raman spectra to derive density and laser-induced plasma 
turbulence levels. ${ }^{73}$ To be able to map out the angular dependence of the scattered light, a multi-channel streaked spectrometer, where the angular position of the detectors can be easily varied, has been implemented on the Nova ten-beam target chamber.

For measurements of the absorption of laser light there is a set of energy balance modules for use on the ten- and the two-beam target chambers.

\section{V) The Next Generation Facility - The NIF}

\section{A) Introduction}

The following definition of a proposed high-energy laser facility is based on ongoing work at LLNL. This facility will be capable of producing $1.8 \mathrm{MJ}$ of temporally shaped laser energy on a variety of targets. This laser system is being developed by the LLNL ICF Program, who provided much of this information.

\section{B) Laser Beams}

The laser will have four separate beam-line bundles, each with a $4 \times 42$ array of beamlets, or 192 beams. The laser is based on the multipass amplifier Nd:YLF configuration where the initial seed laser is generated in a desired temporally shaped pulse. The amplification occurs in a series of multipass and single-pass amplifiers separated by spatial filtering pinholes in increasingly larger diameter sections. The 1- $\omega_{0}$ (i.e, $1.053-\mu \mathrm{m}$ ) wavelength beam arrives at the target chamber with approximately $10 \mathrm{~kJ}$ of energy in a beam $40 \mathrm{~cm}$ square; this assumes a pulse that is temporally a 5-ns square pulse. A variety of other pulse shapes will be available at other energies. The beam is then frequency doubled using a KDP crystal to a 2- $\omega_{0}$ (green) and tripled, to a 3- $\omega_{0}$ (blue) beam for final focusing onto the target. The crystal conversion efficiency will yield approximately $10 \mathrm{~kJ}$ of 3- $\omega_{0}$ energy in the beam to be focused into the target chamber. The 192 beams will be balanced to produce $\leq 8 \%$ rms deviation in power.

The 1- $\omega_{0}$ beam will be of high spatial uniformity; however, the non-linearity of the up conversion to $2 \omega_{0}$ or $3_{0} \omega_{0}$ amplifies any amplitude modulation in the beam. To smooth the beam, a major consideration for directly irradiating a surface where one desires uniformity, several techniques can be used. First, one can introduce a randomness in the phases of the beam with a random phase plate. This creates an effectively smoother intensity distribution by overlapping many small beamlets of random phase created from the large aperture beam. Second, one can split the beam up into several pieces using wedges which allow an effectively flat intensity distribution over the focal spot. Third, one can introduce a bandwidth on the normally monochromatic beam; this will, when combined with a diffraction grating, be used to move the focal spot, resulting in a temporally smoother intensity. 
On NIF, temporal smoothing will also be achieved by overlapping four separate wavelengths, separated by $5-10 \AA$, and smoothing each one by spectral dispersion (adding bandwidth).

The limiting factor in the firing of the beam is the cooling-down time for the large disk amplifiers. This limits a particular laser beamline from firing more than once every eight hours. Diagnostic setup and target alignment will be performed within this time.

\section{C) Beam Pointing, Aiming and Synchronicity}

The beam as it enters the chamber is focused into the target chamber center using an $\mathrm{f} / 8$ lens that will produce a minimum spot size of $500 \mu \mathrm{m}$ in diameter.

The accuracy of the pointing will be $\pm 50 \mu \mathrm{m} \mathrm{rms}$, with a maximum displacement from target chamber center of the beam centerline of $7 \mathrm{~cm}$. The beams come into the chamber in 48 clusters of four beams, with half entering on opposite sides of the midplane. Multiple laser beams will enter on each side of the hohlraum along two concentric cones with cone half-angles of approximately $27^{\circ}$ and $53^{\circ}$, and with $2 / 3$ of the beams on the outer cone and the remaining $1 / 3$ on the inner cone. The pulse shape can be different on the two cones.

\section{D) Laser Pulse Shapes}

There will be a flexible pulse-shaping capability, so that the energy can be delivered in up to a 220-ns shaped pulse with >50:1 intensity contrast between peak and foot of the pulse. Approximately $20 \%$ of the beams can be delayed, independently of each other, by up to $30 \mathrm{~ns}$ after the main beam. This last capability will be useful for providing a source of backlighter $x$-rays.

\section{E) Laser Shot Rate}

NIF will have a capability of 3 shots / 24 hours for 100 shots/year with energy of $100 \mathrm{~kJ}, 35 /$ year for energy of MJ, 10/year for energy of $20 \mathrm{MJ}$.

\section{F) Beam Diagnostics}

The diagnostics of the beam performance that are available are as follows. First, the pulse shape is monitored in the master oscillator for temporal signature and energy. Next the propagated beam can be monitored for the total 1- $\omega_{0}$ energy and the temporal variation of the $1-\omega_{0}$ energy. Further, images can be made of the near and far field of the $1-\omega_{0}$ beam.

The up-converted beam, in either $2 \omega_{0}$ or $3 \omega_{0}$, can be monitored in the chamber for absolute as well as temporal intensity. Further, images of the beam front can be produced in the far or near field. 


\section{References}

1 P. G. Burkhalter et al., Phys. Fluids, 26, 3650 (1983).

2 Seely et al., submitted to Phys Rev. Lett. Zn-like $4141^{\prime}$.

3 Seely et al., submitted to Phys. Rev. E Ho XXXIX density sensitive lines.

4 See, for example, J. Bauche, C. Bauche Arnoult and M. Klapisch in Advances in Atomic Physics , D. R. Bates, Ed. (Academic Press, New York, 1988).

5 B. K. Young et al., Phys. Rev. Lett. 61, 2851 (1988).

6 R. Majoribanks et al., Phys Rev. A 46, R1747 (1992).

7 W. Hsing, B. MacGowan et al. private communication.

8 R. W. Lee, AIP Cunf. Proc. Edited by E. Marmar and J. Terry (American Institute of Physics, New York, NY, 1992) vol. 257.

${ }^{9}$ Perry et al., Phys. Rev. Lett. 67, 3784 (1991).

10 B.K. Young et al., Phys. Rev. Lett. 62, 1266 (1989).

11 L. Da Silva et al., SPIE Proceedings (SPIE) vol. 2012, page 158.

12 T. S. Perry, Phys. Rev. Lett. 67, 3784 (1991).

13 D. R. Kania et al., Phys. Rev A 46, 7853 (1992) and C. A. Back et al., JQSRT 51 (1994).

14 J. N. Nilsen et al., Phys. B 26, L243 (1993).

15 J. N. Nilsen et al., Phys. Rev. A 48, 4682 (1993).

16 J. N. Nilsen et al., Phys. Rev. Lett. 70, 3713 (1993).

17 D. L. Matthews et al., Phys. Reo Lett. 45, 110 (1985).

18 B. J. MacGowan et al., Phys. Rev. Lett. 65, 420 (1990).

19 L. Da Silva et al., Scierice 258, 269 (1992).

20 R. Cauble et al., ICF Quarterly Report 3, 131 (1993).

21 R. Cauble et al., Phys. Rev. Lett. 70, 2102 (1993).

22 B. A. Hammel et al., Phys. Fluids B 5, 2259 (1993).

23 Perry et al., Phys. Rev. Lett. 67, 3784 (1991).

24 L. Da Silva et al., Phys. Rev. Lett. 69, 438 (1992); and D. Guenther, Nature 359, 585 (1992).

25 P. Springer et al., Phys. Rev. Lett. 69, 3735 (1992).

26 J. Wark et al., Phys. Rev. B 35, 9391 (1987).

27 R. R. Whitlock et al., in Shock Compression of Condensed Matter (North Holland, Amsterdam, 1991).

28 J. S. Wark et al., I Appl. Phys. 68, 4531 (1990) and Phys. Rev. B 40, 5705 (1989).

29 P. M. Bell et al., in "Ultrahigh- and High-Speed Photography" and "Videography," SPIE Proc. (SPIE, 1989) vol. 1155.

30. Remington et al., Phys. Fluids B 4, 967 (1992).

31 B. A. Remington et al., Phys. Fluids B 5, 2587 (1992).

32 G. Dimonte and B. A. Remington, Phys. Rev. Lett. 70, 1806 (1993).

33 B. A. Hammel et al., JQSRT 51, number 1 (1994).

34 See, for example, J. Bauche et al. in Advances in Atomic and Molecular Physics, D. Bates and B. Bederson, Eds. (Academic Press, 1988) vol. 23, p. 131 .

35 Eidmann, Phys. Rev. A 41, 3270 (1990).

36 D. Kania, H. Kornblum, B. Hammel, J. Seely, C. Brown, U. Feldman, L. Da Silva, B. MacGowan, D. Montgomery, C. Back, R. Doyas, J. Edwards and R. Lee, Phys. Rev. A 46, 7853 (1992).

37 D. Matthews, E. Campbell, N. Ceglio, G. Hermes, R. Kauffman, L. Koppel, R. Lee, K. Manes, V. Rupert, V. Slivinsky, R. Turner, F. Ze, J. Appl. Phys. 54, 4260 (1983).

38 P. Carroll and G. O'Sullivan, Phys. Rev. A 25, 275 (1982). 
39 K. Eidmann and T. Kishimoto, Appl. Phys. Lett. 49, 377 (1986).

$40 \mathrm{H}$. Nishimura, H. Takabe, K. Kondo, T. Endo, H. Shiraga, K. Sugimoto, T. Nishikawa, Y. Kato, and S. Nakai, Phys. Reo. A 43, 3073 (1991).

41 D. Matthews, E. Campbell, N. Ceglio, G. Hermes, R. Kauffman, L. Koppel, R. Lee, K. Manes, V. Rupert, V. Slivinsky, R. Turner, F. Ze, J. Appl. Phys. 54, 4260 (1983).

42 E. Ott and W. M. Mannheimer, Nuclear Fusion 17, 1057 (1977).

43 D. J. Southwood, Planet. Space Sci. 16, 587 (1968).

44 L. L. Smarr, M. L. Norman and K. A. Winkler, Physica 12D 83 (1984).

45 T. A. Peyser et al., Phys. Fluids B 42, 448 (1992).

46 Handbook of Plasma Physics, M. N. Rosenbluth and R. Z. Sagdeev, Eds., Volume 3: Physics of Laser Plasmas, A. M. Rubenchik and S. Witkowski, Eds. (Elsevier Science Publishers B. V., 1991) Chapter 9 and references therein.

47 F. Ze, L. J. Suter, S. M. Lane, E. M. Campbell, W. C. Mead, J. D. Lindl, M. D. Rosen, D. W. Phillion, C. W. Hatcher, R. P. Drake, J. S. Hildum and K. R. Manes, Comments Plasma Phys. and Controlled Thermonuclear Fusion 10, 33 (1986).

48 D. Montgomery and E. M. Campbell, Comments Plasma Phys. and Controlled Thermonuclear Fusion (1990).

49 E. M. Campbell in: Radiation in Plasınas, B. McNamara, Ed., (1984) vol. I, pp. 579-621.

50 K. R. Manes, V. C. Rupert, J. M. Auerbach, P. Lee and J. E. Swain, Phys. Rev. Lett. 39, 281 (1977).

51 A. G. M. Maaswinkel, K. Eidmann and R. Sigel, Phys. Reo. Lett. 42, 1625 (1979).

52 R. L. Carman, C. K. Rhodes and R. F. Benjamin, Phys. Rev. A 24, 2649 (1981).

53 H. A. Baldis and C. J. Walsh, Phys. Fluids 26, 3426 (1981).

54 H. A. Baldis, J. C. Samson and P. B. Corkum, Phys. Rev. Lett. 41, 1719 (1978).

55 M. D. Rosen, D. W. Phillion, V. C. Rupert, W. C. Mead, W. L. Kruer, J. J. Thomson, H. N. Kornblum, M. N. Rosenbluth, Phys. Rev. Lett. 29, 565 (1979).

56 J. E. Bernard and J. Mdyer, Phys. Fluids 29, 2313 (1986).

57 C. J. Walsh and H. A. Baldis, Phys. Rev. Lett. 48,1483 (1982).

58 J. D. Moody, H. A. Baldis, D. S. Montgomery, K. Estabrook, S. Dixit and C. Labaune, Journal of Fusion Energy 12, 323 (1993).

59 C. J. Walsh, D. M. Villeneuve and H. A. Baldis, Phys. Rev. Lett. 53, 1445 (1984).

60 H. A. Baldis, R. P. Drake, W. L. Kruer, K. G. Estabrook, E. A. Williams and T. W. Johnston, Phys. Rev. Lett. 62, 2829, (1989).

61 C. Joshi, C. E. Clayton, A. Yasuda and F. F. Chen, J. Appl. Phys. 53, 215 (1982).

62 J. A. Stamper, R. H. Lehmberg, A. Schmitt, M. J. Herbst, E. C. Young, J. H. Gardner, and S. P. Obenschain, Phys. Fluids 28, 2563 (1985).

63 P. E. Young, H. A. Baldis, R. P. Drake, E. M. Campbell and K. G. Estabrook, Phys. Rev. Lett. 61, 2336 (1988).

64 J. D. Kilkenny, Laser and Particle Beain 9, 49 (1991).

65 J. D. Kilkenny, Phys. Fluids B2, 1400 (1990); B. A. Remington, et al., Phys. Rev. Lett. 67, 3259 (1991).

66 R. J. Ellis et al., Rev. Sci. Instr. 61, 2759 (1990).

67 J. Balmer et al., Phys Rev. A 40, 330 (1989); T. S. Perry et al., Phys. Rev. Lett. 67, 3784 (1991).

68 J. E. Trebes et al., Phys. Rev. Lett. 68, 588 (1992).

69 B. A. Hammel et al., Rev. Sci. Instrum. 61، 2774 (1990).

70 H. N. Kornblum et al., Rev. Sci. Instrum. 57, 2179 (1986).

71 J. A. Koch et al., Phys. Rev. Lett. (1992).

72 T. Kita et al., Appl. Opt. 22, 512 (1983).

73 R. E. Turner et al., Phys. Rev. Lett. 57, 1725 (1986). 
$1 !$
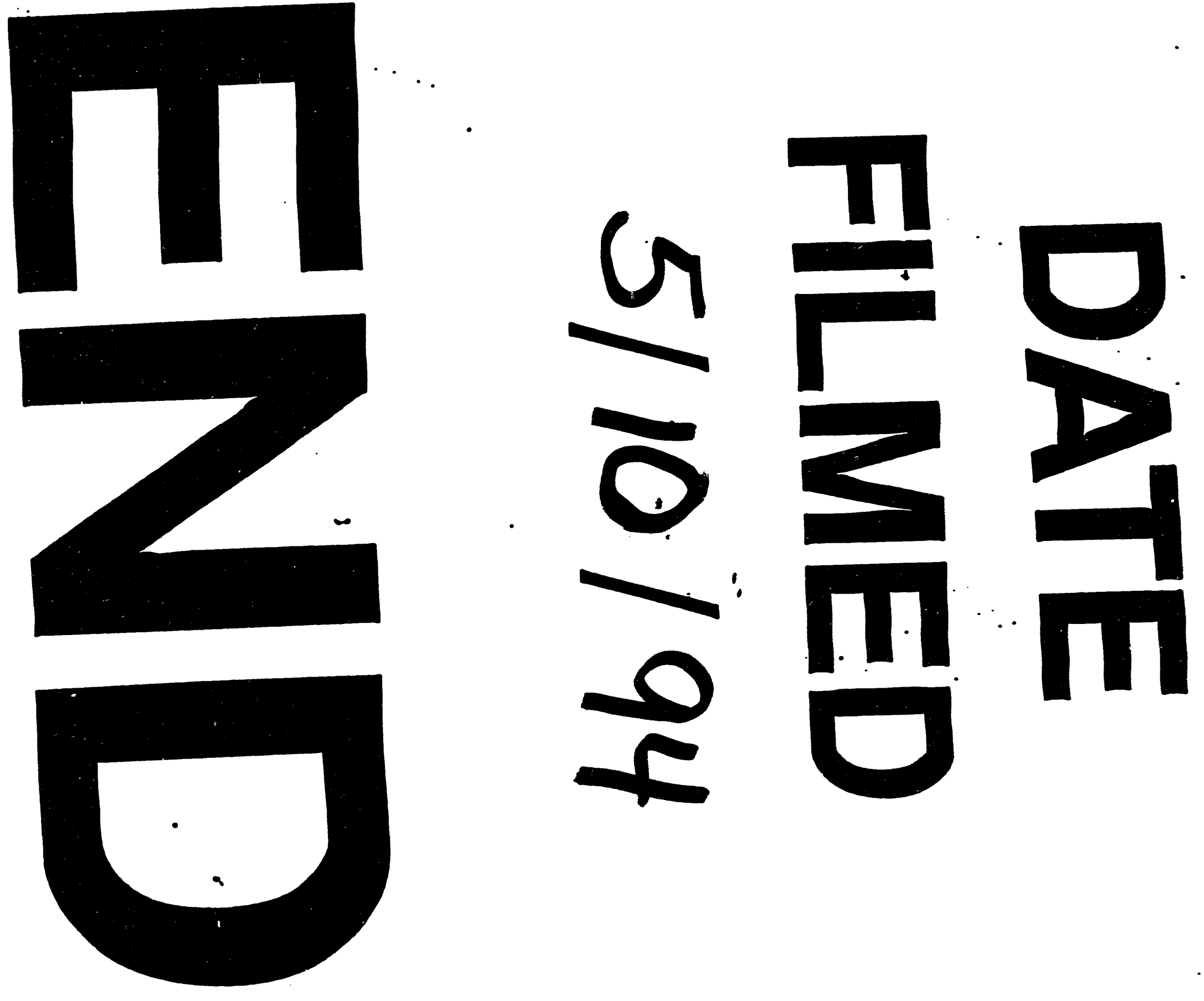
